\title{
Rental Housing Affordability in the Southeast: Data from the Sixth District
}

Ann Carpenter

Federal Reserve Bank of Atlanta
Douglas White

Shimberg Center for Housing Studies, University of Florida

Center for Business and Economic Research, Louisiana State University, Shreveport
Mary Hirt

Federal Reserve Bank of Atlanta

\section{Primary issue:}

The availability of stable and affordable housing in quality neighborhoods provides an opportunity for household economic mobility and a competitive advantage for local jurisdictions. The Southeast, as in other areas of the country, has experienced a persistent affordable housing shortage since the Great Recession. This is due in part to historically low homeownership rates, rents that have increased at a faster rate than income, and the loss of subsidized and unsubsidized rental units due to abandonment and conversion.

\section{Key findings:}

More than two-thirds (69 percent) of low-income renter households pay over 30 percent of their income on housing across the Southeast, making them "cost burdened." This paper provides similar data for states, metropolitan and micropolitan areas, and cities. Based on the data, cost-burdened households are in rural areas, small towns, suburbs, and large urban centers. The finding is not surprising, given there is a shortage of more than 1.2 million units of housing that is affordable and available to households making 50 percent or less of area median income in the six states the Atlanta Fed covers (Alabama, Florida, Georgia, Louisiana, Mississippi, and Tennessee).

\section{Takeaways for practice:}

State and local leaders and housing stakeholders across the Southeast are working to tackle this issue. We offer examples of strategies for public agencies, nonprofits, philanthropies, and the private sector to increase the affordable rental supply, preserve existing affordable units, and stabilize renter households at risk of eviction. Generally, this includes a clearly articulated problem statement and vision, more dedicated state and local resources, reduced barriers to development such as exclusionary land use and zoning policies, and tenant protections.

\section{The Federal Reserve Bank of Atlanta's Community \& Economic Development (CED) Discussion Paper Series} addresses emerging and critical issues in community development. Our goal is to provide information on topics that will be useful to the many actors involved in community development-governments, nonprofits, financial institutions, and beneficiaries. Find more research, use data tools, and sign up for email updates at frbatlanta.org/commdev. 


\title{
Rental Housing Affordability in the Southeast: Data from the Sixth District
}

\begin{abstract}
:
Housing data are available for most large metropolitan regions in the Atlanta Fed's Southeast region. However, many midsized metropolitan, micropolitan, and nonmetro areas lack detailed data on rental housing affordability and housing supply needs by income level. These data are important for state and local governments, affordable housing developers, and housing advocates to inform housing policy. Therefore, the Atlanta Fed partnered with the Shimberg Center at the University of Florida to analyze census data using a methodology developed for Shimberg's periodic Rental Market Study for the state of Florida (Shimberg Center for Housing Studies, 2013, 2016). This paper covers the six states that are fully or partially in the Atlanta Fed's District: Alabama, Florida, Georgia, Louisiana, Mississippi, and Tennessee.
\end{abstract}

In this paper, we provide a regional snapshot of housing affordability and the availability of affordable rental housing units at several scales for the Atlanta Fed's District, using data from the 2015 American Community Survey (ACS). We include figures for city, metropolitan, and state areas as well as regional figures for nonmetro areas. We segment the data by household income using the area median income (AMI) of each respective region. We provide estimates for renter households within five major income brackets: extremely low income (0 to 30 percent AMI), very low income (30.01 to 50 percent $\mathrm{AMI}$ ), low income (50.01 to 80 percent $\mathrm{AMI}$ ), moderate income (80.01 to 120 percent $\mathrm{AMI}$ ), and upper income (more than 120 percent $\mathrm{AMI})$.

We use two measures of housing affordability: 1) the share of cost-burdened households and 2) affordable and available rental housing supply. Metrics include the percent of cost-burdened renter households (people who pay more than 30 percent of their income on housing) and extremely costburdened renter households (people who pay more than 50 percent of their income on housing). Metrics also include the deficit or surplus in rental units that are both available and affordable to households at each of the above area median-income brackets. These measures tend to correlate, with high percentages of cost-burdened households associated with significant deficits in affordable and available units for low- and moderate-income households.

Our results demonstrate the widespread lack of affordable housing in large metropolitan areas, small and midsized regions, and nonmetro regions throughout the Southeast. Although large metros such as Atlanta, Miami, Nashville, and New Orleans have received attention for the large increases in 
rent and subsequent affordability crises, markets such as Cape Coral and Orlando, Florida, and Savannah, Georgia, have similar or even higher levels of rent-burdened households. We also show that extremely low- and very low-income households are disproportionately cost burdened.

JEL classification: H53, R21, R31, R38

Key words: rental housing, affordable housing, low-income housing, housing cost burden

https://doi.org/10.29338/dp2018-02

\section{About the Authors:}

Ann Carpenter is a senior community and economic development adviser at the Federal Reserve Bank of Atlanta, specializing in housing and neighborhood revitalization. Her recent work includes studies on land contracts and strategies to increase the production of mixed-income housing. Prior to joining the Atlanta Fed, Carpenter was a senior research associate at the Georgia Tech Research Institute (GTRI). There, she specialized in the areas of community resilience and emergency management planning. Carpenter earned a bachelor's degree in architecture from the University of Michigan and master's and doctorate degrees in city and regional planning from Georgia Tech. She is a member of the American Institute of Certified Planners (AICP).

Doug White is the director of the Center for Business and Economic Research at Louisiana State University, Shreveport and a researcher affiliated with the Shimberg Center for Housing Studies at the University of Florida. He contributes to the development of the Florida Housing Data Clearinghouse and coauthors the annual publication The State of Florida's Housing. He has degrees from the University of Michigan (bachelor of science in chemical engineering), the University of Louisville (MBA), and Florida State University (master of science in economics), where he has also completed course work toward a $\mathrm{PhD}$ in economics.

Mary Hirt is a research analyst for the Federal Reserve Bank of Atlanta's community and economic development group. She supports the team through research, data analysis, and communication and outreach efforts. Hirt holds a bachelor of arts in international studies with minors in social work and urban studies from the University of Michigan. After moving to Atlanta, Hirt earned a master's degree in city and regional planning from Georgia Institute of Technology where her research focused on housing and community development. Before pursuing her graduate degree, Hirt worked for the University of Michigan's Institute for Social Research in Ann Arbor and the Center for Civic Innovation in Atlanta.

Acknowledgments: The authors thank Chris Cunningham, Karen Leone de Nie, Eileen Divringi, Bill O'Dell, and Anne Ray for their feedback on earlier drafts of this report. The views expressed here are the authors'

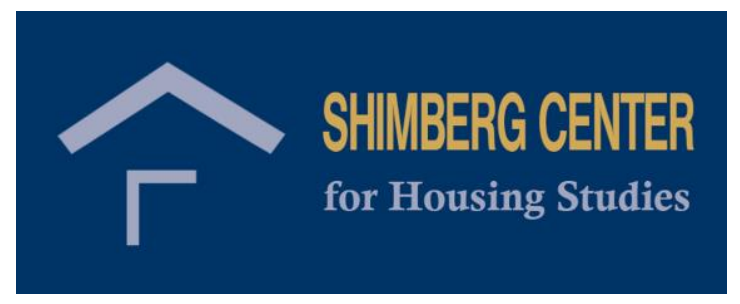


and not necessarily those of the Federal Reserve Bank of Atlanta or the Federal Reserve System. Any remaining errors are the authors' responsibility.

The Shimberg Center for Housing Studies at the University of Florida provided data analysis support for this paper. The Shimberg Center and the Federal Reserve Bank of Atlanta prepared the paper jointly as part of the Atlanta Fed's Community and Economic Development Discussion Paper series.

Comments to the authors are welcome at ann.carpenter@atl.frb.org, douglas.white@lsus.edu, and mary.hirt@atl.frb.org. 


\section{Acronyms}

ACS (U.S. Census Bureau's) American Community Survey

AMI Area median income

CBSA Core-based statistical area

ELI Extremely low income

HUD U.S. Department of Housing and Urban Development

LI Low income

MSA Metropolitan statistical area

PUMA Public use microdata area

PUMS Public use microdata sample

TIGER Topologically integrated geographic encoding and referencing database

VLI Very low income

$\mu \mathrm{SA} \quad$ Micropolitan statistical area 


\section{Background}

Nestled in the booming "Sun Belt," the Southeast has attracted residents for decades due to a relatively low cost of living and plentiful economic opportunities. However, the region has recently struggled along with the rest of the United States as housing costs have increased and demand for rental housing has grown.

Over time, affordability as measured by the share of cost-burdened renter households (those paying more than 30 percent of their income on housing) has varied between 49 percent and 53 percent at the national level (see figure 1). States in the Atlanta Fed's District range from relatively affordable for renters (Tennessee) to among the least affordable in the country (Florida). In most states, the share of cost-burdened households peaked around 2011 and has declined since then, indicating rental affordability has improved as household incomes have begun to rise modestly. However, the share of cost-burdened households in Louisiana has actually increased in the ensuing years. Within each of these states, metropolitan and city affordability ranges greatly.

\section{Figure 1: Percent of Renter Households That Are Cost Burdened, 2005-16, by State}

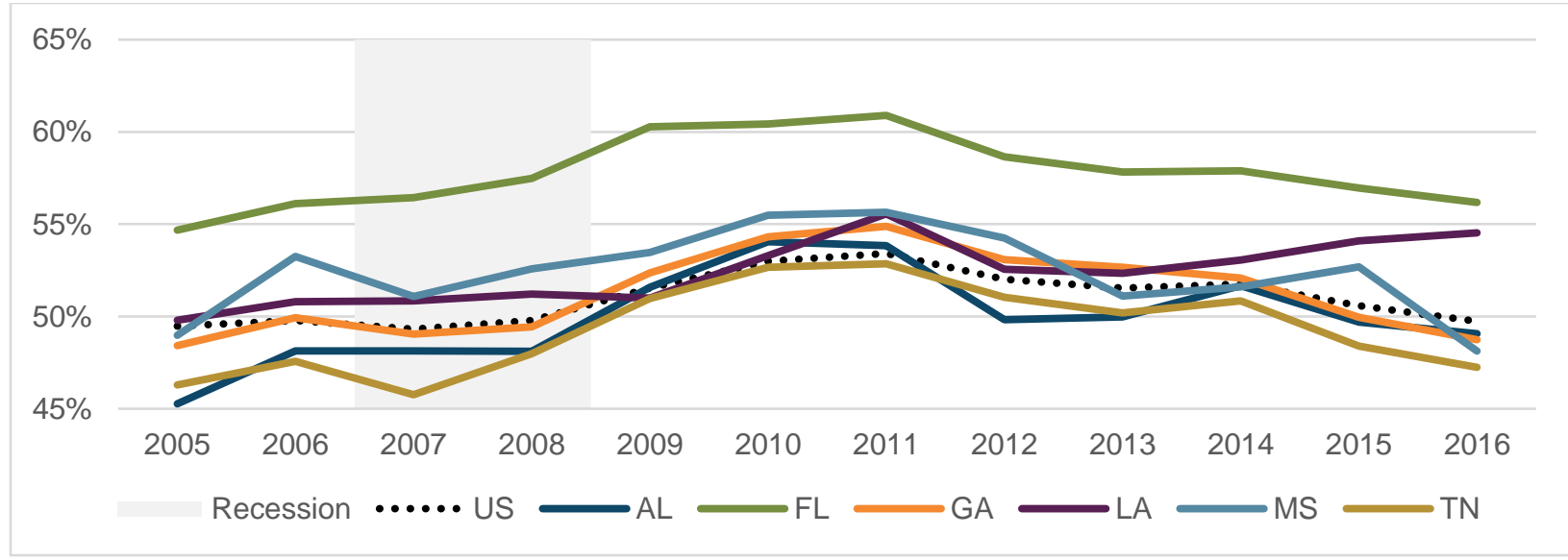

Source: U.S. Census Bureau's American Community Survey 1-Year Estimates

A lack of affordable housing is associated with housing instability; households paying a larger share of their income on housing have fewer resources to weather an economic shock such as a health emergency or job loss. Evictions in each of the Atlanta Fed's District states have remained relatively constant since before the housing crisis. Rates range from a high of 4.7 percent of all renter households evicted in 2016 in Georgia to a low of 1.8 percent in Alabama (see figure 2). 


\section{Figure 2: Statewide Eviction Rates in the Southeast (Percent of Renter Households Evicted by Year)}

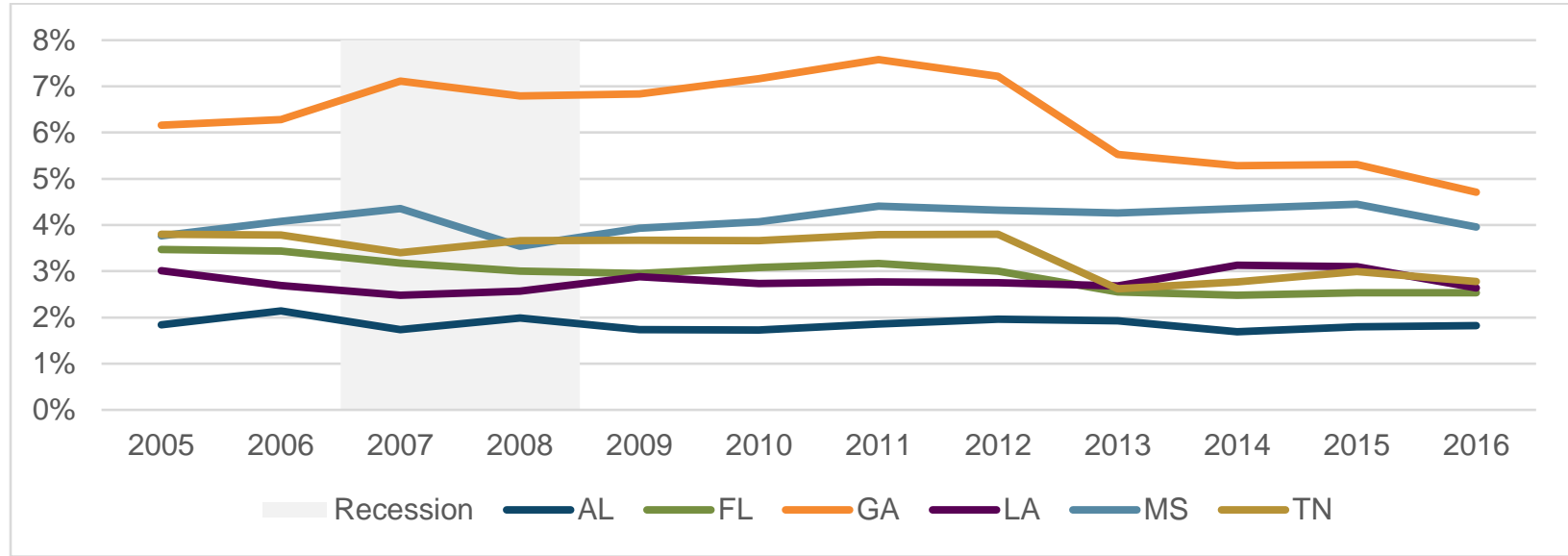

Source: EvictionLab.org

Large differences in eviction rates by state point to the different data sources used across the data set and differing levels of tenant protection ${ }^{1}$ as well as affordability issues, including both affordable housing supply and the availability of jobs that pay a living wage. As shown in figure 3 , eviction-filing rates tend to vary even more significantly by state within the Southeast, with Georgia among the top states in the United States. Eviction filings are just as likely to damage a renter's economic situation and limit future housing opportunities, since background checks performed by landlords include filings as well as dispossessory actions.

\section{Figure 3: 2016 Eviction Filing Rate versus Eviction Rate by Southeastern State}

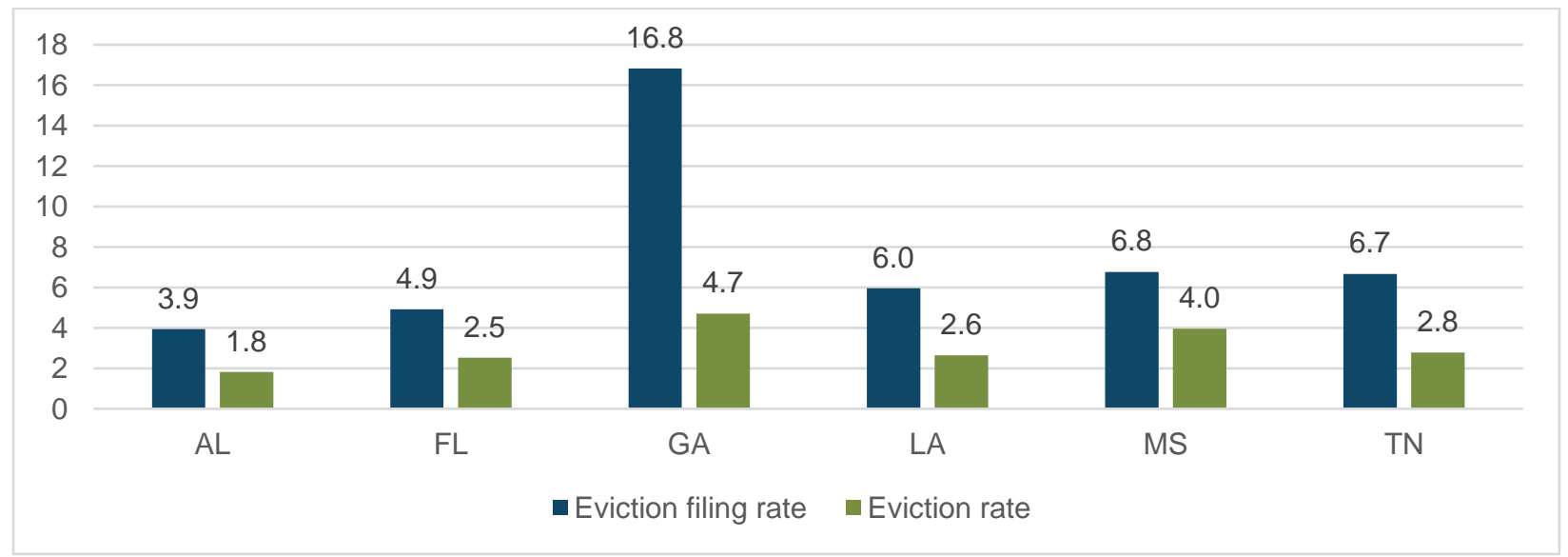

Source: EvictionLab.org

\footnotetext{
${ }^{1}$ For instance, in Georgia, Mississippi, and Alabama, landlords are not prevented from taking retaliatory actions against a tenant who asserts his or her rights under the law (http://lawatlas.org/datasets/state-landlord-tenantlaws-1499878846).
} 
There has also been an observed decline in lower-cost rented units in the Southeast, particularly in central cities (Immergluck, Carpenter, \& Lueders, 2016), with cities such as Nashville and Atlanta losing more than a thousand units per year that had monthly rents of $\$ 750$ or below. Further, an estimated 59,255 currently affordable rental units in the Atlanta Fed's District built with subsidies in exchange for rent limits are at risk of reverting to market rate in the next five years without additional investment to preserve affordability requirements (National Housing Preservation Database, 2018). Given the above data, rental housing affordability is a growing concern among many stakeholder groups in the Southeast. This report provides detailed data at various scales to inform policy and activate resources needed to provide affordable rental housing options. We also provide figures on the gap in rental housing units by calculating the number of units both affordable and available to households at several income categories. Housing data are available for most large metropolitan regions in the Southeast region covered by the Federal Reserve Bank of Atlanta; however, many midsized metropolitan, micropolitan, and nonmetro areas lack detailed data on housing affordability and housing supply needs. Therefore, the Atlanta Fed partnered with the Shimberg Center at the University of Florida to analyze census data using a methodology developed for Shimberg's periodic Rental Market Study for the state of Florida (Shimberg Center for Housing Studies, 2013, 2016). Our paper covers all six states that are fully or partially in in the Atlanta Fed's District: Alabama, Florida, Georgia, Louisiana, Mississippi, and Tennessee.

\section{Data}

In this paper, we provide a regional snapshot of rental housing affordability and the availability of affordable rental housing units at several scales for the Atlanta Fed's District, using the U.S. Census Bureau's 2015 American Community Survey (ACS) 1-Year public use microdata sample (PUMS). The ACS is the yearly population and housing survey that replaced the Decennial Census's detailed long-form questionnaire. The ACS surveys a sample of approximately 3.5 million people per year and provides relatively current information on housing and population trends. The census weights the sample data to produce full estimates by individual geography and for the United States.

The census releases the ACS data in prepared tables that describe population and housing data at multiple geographical levels, that is, national, state, county, and subcounty levels. While these tables are useful, a user cannot fully customize them. Therefore, the census also releases the ACS data in public use microdata sample (PUMS), with individual, de-identified records for individuals and housing units. One advantage to the PUMS data is the ability to create customized cross tabs, but this flexibility is limited. To protect privacy, the census releases the data with a geographic identifier known as a public use microdata area (PUMA). ${ }^{2}$ Each PUMA contains at least 100,000 people and is contained within a state; however, PUMAs do not necessarily match other census geographies. To ensure an area contains the required 100,000 residents, PUMAs combine multiple tracts, counties, and even split counties depending on the state and its population density. The fact that PUMA geography is different from the standard census tract, county, and metropolitan statistical areas (MSAs) routinely used by the census

\footnotetext{
2 Each state's Data Center last defined PUMAs in 2010 using census guidelines.
} 
means that it is not always possible to provide cross tabulations at the level of common census boundaries.

\section{Methodology}

The goal of this paper is to measure levels of cost burden among renter households as well as rental housing affordability and availability by income category in subregions of the Atlanta Fed's District. To keep these figures consistent with federal affordable housing income standards and rents, we mimic the U.S. Department of Housing and Urban Development (HUD) methodology for calculating area median income (AMI), household size-adjusted income, and bedroom size-adjusted rent. We also use HUD's affordability standard, in which households should spend no more than 30 percent of their income on housing.

HUD measures its AMI figures at the MSA level. Therefore, the first step is to re-create MSAs by combining PUMAs. We then assign renter households to each MSA. Where possible, the best approach for creating MSAs is first to isolate individual counties in each state and combine these counties to form the MSA. Again, while it is possible to create many MSAs using this method, in certain cases the difference between the PUMA geography and the standard census geography requires either the addition or subtraction of certain counties. In rural areas, because of low population, many counties often are included in PUMAs that cross MSA boundaries and thus, for this analysis, MSAs must be combined with other geographies, such as micropolitan statistical areas or nonmetro counties.

We assigned PUMAs as closely as possible to MSAs as well as to cities. A total of 29 cities and 99 larger regions created for analysis by combining PUMAs are shown in figures 4 and 5 . Most PUMAs in the Atlanta Fed's District conformed to county boundaries, but Marion County, Alabama, is split between two PUMAs. 
Figure 4: PUMAs and Combined PUMA Regions Used for Analysis

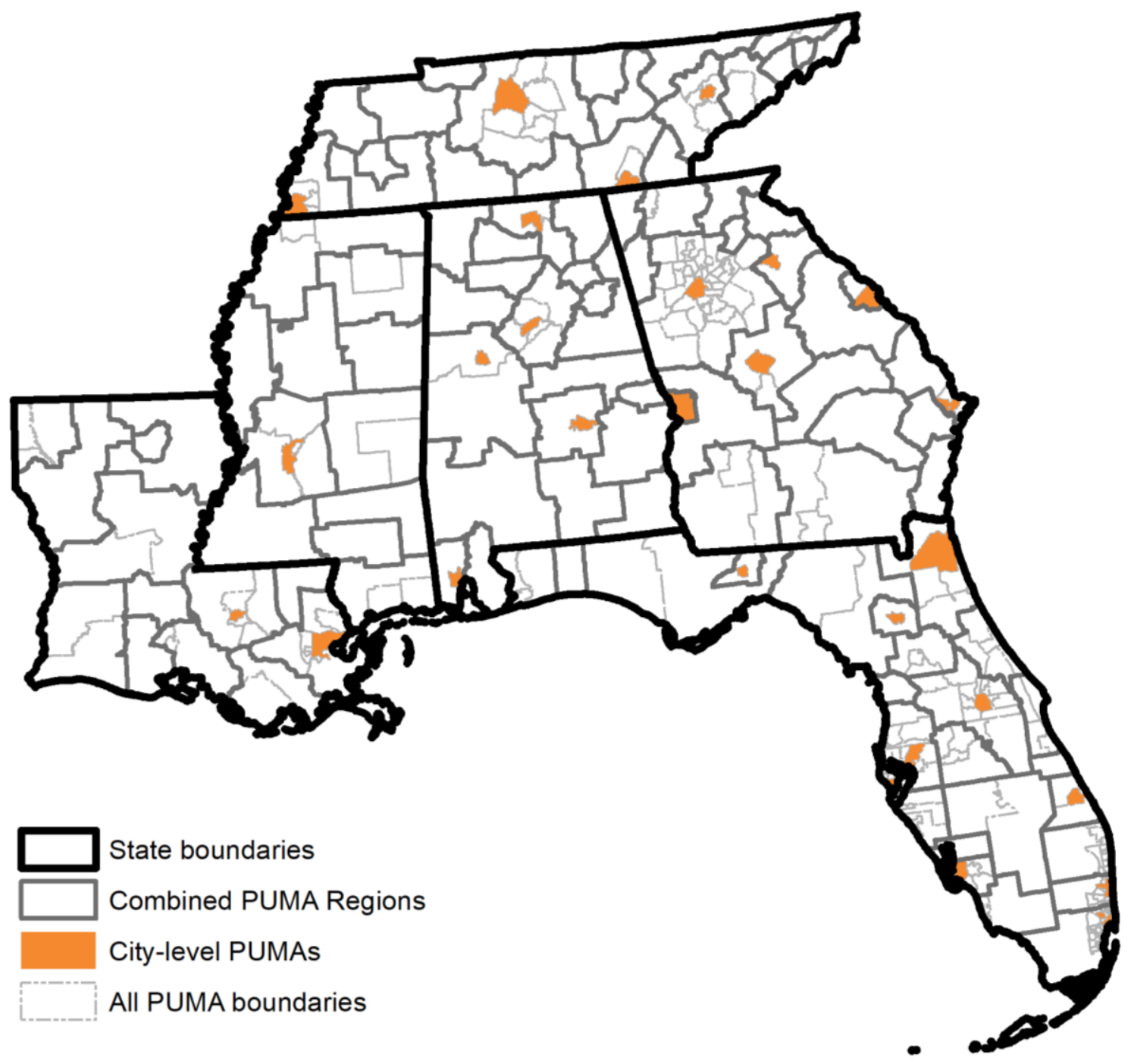

Source: U.S. Census Bureau's topologically integrated geographic encoding and referencing (TIGER) data 
Figure 5: Combined PUMA Regions Used for Analysis Compared to Census Core-Based Statistical Areas (MSAs and Micropolitan Statistical Areas)

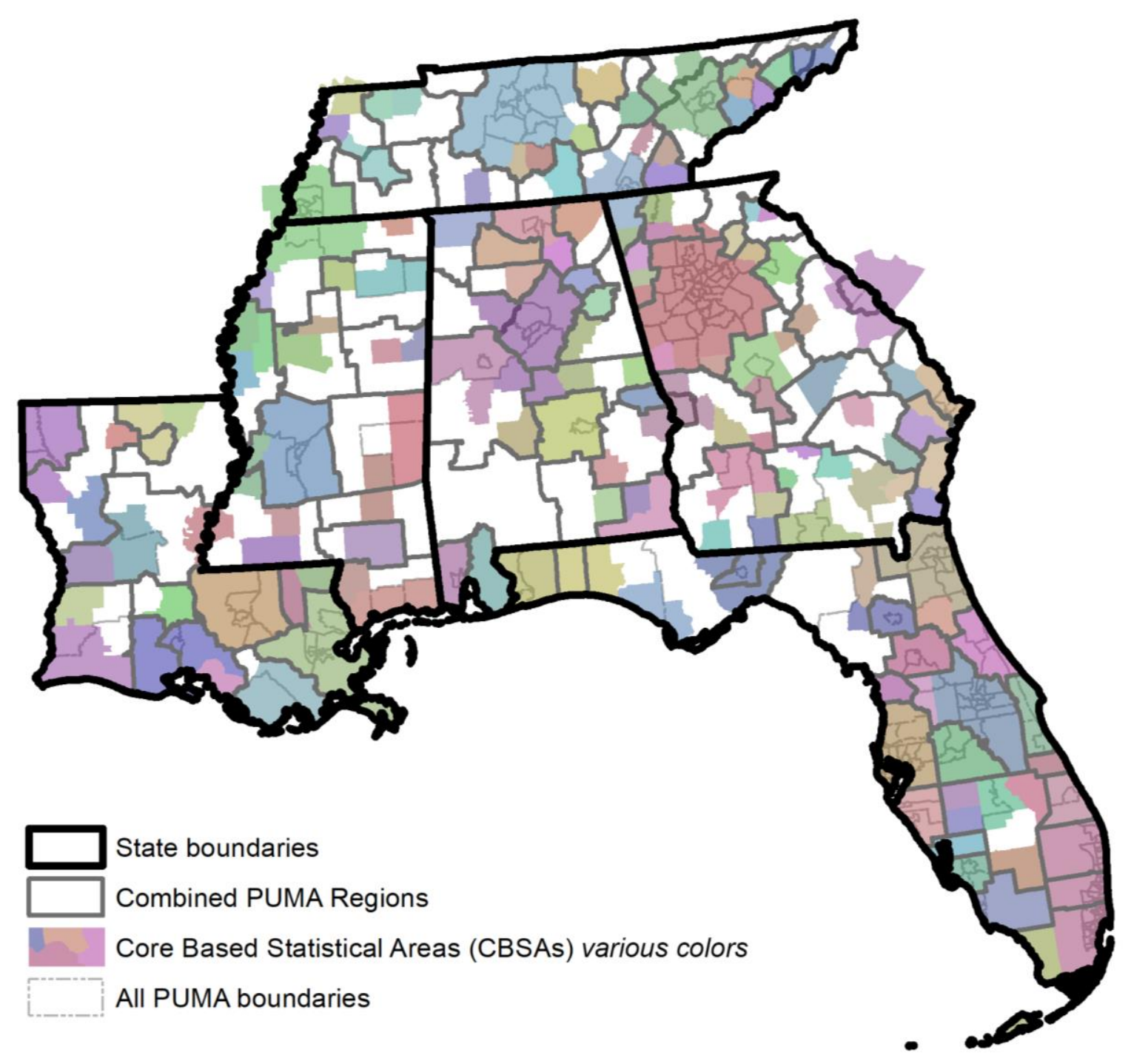

Source: U.S. Census's Bureau topologically integrated geographic encoding and referencing (TIGER) data

Once we created MSA and other combined PUMA regions, the next step was to calculate the area median income (AMI) of each MSA using the ACS data. We used the AMI to assign households to an income category and housing units to an affordability category. The AMI was calculated for family households only (two or more people residing together and related by birth, marriage, or adoption) and by calculating the median of the reported income of these households across the MSA. Using families instead of households mirrored HUD's approach to calculating the AMI. Since the MSAs we created did not necessarily match census MSAs, we performed a check to determine if the AMI was reasonable for all counties in the regions we created. We then compared MSA area median income to county-level AMI data reported by HUD for each constituent county. Many of the county AMIs were reasonably similar to 
the MSA, and therefore the MSA AMI was usable. However, we sometimes found AMIs that were different enough to recommend further investigation.

In cases where MSAs are made up of multiple PUMAs (for example, PUMAs that include nonMSA counties or counties belonging to another micropolitan or metropolitan area), an individual PUMA AMI in some cases was a better match for the counties in that PUMA, based on HUD's county-level AMI. Table 1 shows an example of this. The Augusta-Richmond County, Georgia-South Carolina, MSA included three PUMAs: 4000, 4100, and 4200. The calculated AMI for the Augusta MSA based on the income of the families in these PUMAs is $\$ 55,470$. This AMI is significantly higher than the HUD AMI for the counties in PUMA 4200. If the MSA AMI is found by calculating PUMA 4000 and 4100 together and 4200 on its own, the resulting MSA AMI better matches the underlying counties. Eight total MSA regions were split in two using a similar methodology: in Alabama, Birmingham; in Georgia, Augusta and Columbus; in Louisiana, Lafayette and Monroe; and in Tennessee, Jackson, Johnson City, and Knoxville. Despite these corrections for area median income, it was not always possible to eliminate all differences between HUD's county-level AMI and our calculated AMI at the PUMA level using PUMS data.

\section{Table 1: Augusta-Richmond County, Georgia-South Carolina, MSA AMI Comparison}

\begin{tabular}{|l|c|c|c|c|}
\hline County & PUMA & $\begin{array}{c}2015 \text { HUD } \\
\text { County- } \\
\text { Level AMI }\end{array}$ & $\begin{array}{c}2015 \text { ACS Calculated } \\
\text { AMI for PUMAs 4000, } \\
4100, \text { and 4200 }\end{array}$ & $\begin{array}{c}2015 \text { ACS Calculated AMI } \\
\text { for PUMA 4000 and 4100 } \\
\text { versus PUMA 4200 }\end{array}$ \\
\hline Richmond County, GA & 4000 & $\$ 59,100$ & $\$ 55,470$ & $\$ 60,076$ \\
\hline Columbia County, GA & 4100 & $\$ 59,100$ & $\$ 55,470$ & $\$ 60,076$ \\
\hline Burke County, GA & 4200 & $\$ 59,100$ & $\$ 55,470$ & $\$ 40,651$ \\
\hline Glascock County, GA & 4200 & $\$ 51,300$ & $\$ 55,470$ & $\$ 40,651$ \\
\hline Hancock County, GA & 4200 & $\$ 35,400$ & $\$ 55,470$ & $\$ 40,651$ \\
\hline Jefferson County, GA & 4200 & $\$ 36,900$ & $\$ 55,470$ & $\$ 40,651$ \\
\hline Jenkins County, GA & 4200 & $\$ 34,100$ & $\$ 55,470$ & $\$ 40,651$ \\
\hline Lincoln County, GA & 4200 & $\$ 45,900$ & $\$ 55,470$ & $\$ 40,651$ \\
\hline McDuffie County, GA & 4200 & $\$ 59,100$ & $\$ 55,470$ & $\$ 40,651$ \\
\hline Taliaferro County, GA & 4200 & $\$ 38,800$ & $\$ 55,470$ & $\$ 40,651$ \\
\hline Warren County, GA & 4200 & $\$ 40,400$ & $\$ 55,470$ & $\$ 40,651$ \\
\hline Washington County, GA & 4200 & $\$ 49,300$ & $\$ 55,470$ & $\$ 40,651$ \\
\hline Wilkes County, GA & 4200 & $\$ 39,100$ & $\$ 55,470$ & $\$ 40,651$ \\
\hline
\end{tabular}

Sources: U.S. Department of Housing and Urban Development, authors' tabulations of U.S. Census Bureau's 2015 American Community Survey public use microdata sample (PUMS) data

Appendix A shows each combined PUMA region (MSA, micropolitan area, or nonmetro area) along with the counties included and the AMI used for calculations. The tables in appendix $A$ also document situations such as the one above by indicating AMI in parts (that is, "Part 1," "Part 2") with a list of counties included in each part.

We used the MSA AMI to place renter households in the following income categories: extremely low income (0 to 30 percent $A M I)$, very low income (30.1 to 50 percent $A M I)$, low income (50.01 to 80 
percent $\mathrm{AMI}$ ), moderate income (80.01 to 120 percent $\mathrm{AMI}$ ), and upper income (more than 120 percent AMI). Similar to HUD's income limit categories, the income category for renter households is also based on household size. HUD bases affordable rent for each household size on the AMI for a four-person family. The base AMI adjusts down for households with fewer than four people and adjusts up for households with more than four people. ${ }^{3}$ HUD uses these AMIs to set income limits for extremely low-, very low-, and low-income families. Using reported household income and the reported number of people in the household from the ACS PUMS data, we placed renter households in the appropriate income category by dividing their reported income by the household size-appropriate AMI. ${ }^{4} \mathrm{We}$ calculated each household's reported rent costs as a percentage of total reported household income to determine whether a household was cost burdened (paying more than 30 percent of household income on rent) or extremely cost burdened (paying more than 50 percent of household income on rent). Households with zero or negative income were not considered cost burdened.

HUD's formula also prescribes the income needed to rent a unit based on the number of bedrooms and the MSA area median income. Using this formula and our calculated AMI, we then found the income needed to rent each rental housing unit reported in the ACS PUMS data and placed those units into appropriate affordability categories. First, we found the bedroom-weighted income needed. We did this using the ACS reported number of bedrooms and the formula created by HUD. ${ }^{5}$

Using the American Community Survey housing unit data, we then calculated whether a unit is affordable. We did this calculation by comparing the sum of the ACS reported rent costs, electric costs, fuel costs, gas costs, and water costs to the appropriate bedroom-weighted income needed. We also assume that these summed costs cannot be more than 30 percent of the renter's income. This allows us to place the unit in one of three categories: affordable at 30 percent AMI, affordable at 50 percent AMI, or affordable at 80 percent AMI.

The result is a database of renters and rental units by appropriate AMI category. Comparing the number of renters to number of rental units in each of the above affordability categories tells us whether there is a surplus or shortage of affordable units for that income category. The shortage of units is often referred to as the housing gap. Our analysis goes a step farther in measuring affordability. If we had perfect sorting in the market, renters would only rent units corresponding to their income level, such that renters with 30 percent or less AMI would rent units affordable at 30 percent AMI, renters with 50 percent $A M I$ would rent units affordable at 50 percent $A M I$, and so on. However, renters often rent down, so a renter with 80 percent $\mathrm{AMI}$ may rent a unit that is affordable at 50 percent or a

\footnotetext{
${ }^{3}$ The adjustments are as follows: one person is 70 percent AMI; two people are 80 percent AMI; three people are 90 percent AMI; five people are 109 percent AMI; six people are 116 percent AMI; and seven people are 124 percent AMI.

${ }^{4}$ Note we did not remove college students in nonfamily households for this analysis, thus, the number of costburdened households may include this population.

${ }^{5}$ For zero bedrooms, income needed is 70 percent AMI; for one bedroom, income needed is 75 percent $\mathrm{AMI}$; for two bedrooms, income needed is 90 percent AMI; for three bedrooms, income needed is 104 percent AMI; for four bedrooms, income needed is 116 percent $\mathrm{AMI}$; for five bedrooms, income needed is 128 percent $\mathrm{AMI}$; for six bedrooms, income needed is 140 percent AMI; and for seven-plus bedrooms, income needed is 140-plus (12* number of additional bedrooms) percent AMI.
} 
renter with 50 percent $A M I$ may rent a unit affordable at 30 percent $A M I$, and so forth. They may also crowd into units that are smaller than HUD deems sufficient for their family size. While this might make financial sense for the higher-income renter by saving money on rent, that lower-cost unit is then not available for a renter with lower income. Thus, we measured the rental units occupied by rental households with the appropriate income level for that unit. We then compared the rental units in the ACS by looking at both the appropriate affordability level of the unit and the ACS reported renter household income. Those units occupied by households with the appropriate income we consider available. Comparing the number of renters with the available units gives a truer count of the housing gap in each market. Although the income categories are helpful for planning purposes, sorting may also occur within these relatively broad segments. For example, many units affordable at 30 percent AMI and below (renters with extremely low incomes) may not be affordable to the significant share of households that make at or near zero dollars in income.

\section{Results: State-Level Data}

The findings for the number and share of households that are cost burdened (households that pay more than 30 percent of household income on rent) and extremely cost burdened (households that pay more than 50 percent of income on rent) varied quite a bit at the state, MSA, and city levels among geographies in the Southeast. Across all six states in the Atlanta Fed's District, approximately 3 million renter households (47 percent) are cost burdened and an additional 1.6 million owner-occupied households (22 percent) are cost burdened. Of these, 1.5 million renter households and 1.1 million owner-occupied households are extremely cost burdened. Among low-income households in all six states, 2.7 million households (69 percent) are cost burdened. The hardship is particularly great for lower-income households, which, after paying rent, have fewer dollars to devote to other households needs such as transportation, child care, and health care.

As shown in table 2, Florida has the highest percentage of cost-burdened renter households in every income category and among all renter households in general (51 percent). Florida also has a notably larger share of cost-burdened renter households at moderate and upper incomes. Alabama, Mississippi, and Tennessee have relatively lower rates of cost-burdened renter households compared to other Atlanta Fed District states; Georgia and Louisiana fall in the middle. 
Table 2: Number (and Percent) of Renter Households That Are Cost Burdened (Rent Is $>\mathbf{3 0} \%$ Household Income) by Income Category and by State

\begin{tabular}{|c|c|c|c|c|c|c|}
\hline & $\begin{array}{l}\text { Extremely } \\
\text { Low Income } \\
\text { (30\% AMl or } \\
\text { less) }\end{array}$ & $\begin{array}{l}\text { Very Low } \\
\text { Income } \\
\text { (30.01 to } \\
50 \% \text { AMI) }\end{array}$ & $\begin{array}{c}\text { Low } \\
\text { Income } \\
\text { (50.01 to } \\
80 \% \text { AMI) }\end{array}$ & $\begin{array}{l}\text { Moderate } \\
\text { Income } \\
\text { (80.01 to } \\
120 \% \mathrm{AMI} \text { ) }\end{array}$ & $\begin{array}{l}\text { Upper } \\
\text { Income } \\
\text { (More than } \\
120 \% \text { AMI) }\end{array}$ & $\begin{array}{c}\text { All Renter } \\
\text { House- } \\
\text { holds }\end{array}$ \\
\hline $\mathrm{AL}$ & $\begin{array}{c}114,152 \\
(70 \%)\end{array}$ & $\begin{array}{c}78,398 \\
(69 \%) \\
\end{array}$ & $\begin{array}{c}40,649 \\
(36 \%)\end{array}$ & $\begin{array}{c}8,862 \\
(9 \%) \\
\end{array}$ & $\begin{array}{c}1,339 \\
(1 \%)\end{array}$ & $\begin{array}{c}243,400 \\
(41 \%)\end{array}$ \\
\hline $\mathrm{FL}$ & $\begin{array}{c}363,023 \\
(74 \%)\end{array}$ & $\begin{array}{c}384,804 \\
(87 \%)\end{array}$ & $\begin{array}{c}401,946 \\
(70 \%)\end{array}$ & $\begin{array}{c}178,150 \\
(34 \%)\end{array}$ & $\begin{array}{c}58,973 \\
(9 \%)\end{array}$ & $\begin{array}{c}1,386,896 \\
(51 \%)\end{array}$ \\
\hline GA & $\begin{array}{c}227,993 \\
(73 \%)\end{array}$ & $\begin{array}{c}198,685 \\
(82 \%)\end{array}$ & $\begin{array}{c}149,298 \\
(54 \%)\end{array}$ & $\begin{array}{c}37,151 \\
(14 \%)\end{array}$ & $\begin{array}{l}7,653 \\
(3 \%)\end{array}$ & $\begin{array}{c}620,780 \\
(45 \%)\end{array}$ \\
\hline LA & $\begin{array}{c}112,478 \\
(69 \%)\end{array}$ & $\begin{array}{c}92,169 \\
(79 \%)\end{array}$ & $\begin{array}{c}57,763 \\
(48 \%)\end{array}$ & $\begin{array}{c}15,560 \\
(15 \%)\end{array}$ & $\begin{array}{c}3,319 \\
(3 \%)\end{array}$ & $\begin{array}{c}281,289 \\
(46 \%)\end{array}$ \\
\hline MS & $\begin{array}{c}56,086 \\
(63 \%)\end{array}$ & $\begin{array}{c}45,887 \\
(70 \%)\end{array}$ & $\begin{array}{c}37,543 \\
(54 \%)\end{array}$ & $\begin{array}{c}11,273 \\
(18 \%)\end{array}$ & $\begin{array}{c}1,956 \\
(3 \%)\end{array}$ & $\begin{array}{c}152,745 \\
(42 \%)\end{array}$ \\
\hline TN & $\begin{array}{c}147,175 \\
(71 \%)\end{array}$ & $\begin{array}{c}110,797 \\
(73 \%)\end{array}$ & $\begin{array}{l}83,540 \\
(44 \%)\end{array}$ & $\begin{array}{c}19,192 \\
(13 \%)\end{array}$ & $\begin{array}{c}4,629 \\
(3 \%)\end{array}$ & $\begin{array}{c}365,333 \\
(42 \%)\end{array}$ \\
\hline
\end{tabular}

Source: Authors' tabulations of U.S. Census Bureau's 2015 American Community Survey public use microdata sample (PUMS) data

A large majority of extremely low- and very low-income renter households (those earning 50 percent or less of AMI) are cost burdened or extremely cost burdened in every state in the Atlanta Fed's District, ranging from 66 percent in Mississippi to 80 percent in Florida (see figure 6). At this level of income, Florida has the largest share of households that are extremely cost burdened and cost burdened combined, and a particularly larger share of households are extremely cost burdened.

\section{Figure 6: Percent of Extremely Low-Income and Very Low-Income Renter Households} ( $<50 \%$ AMI) That Are Cost Burdened and Extremely Cost Burdened by State

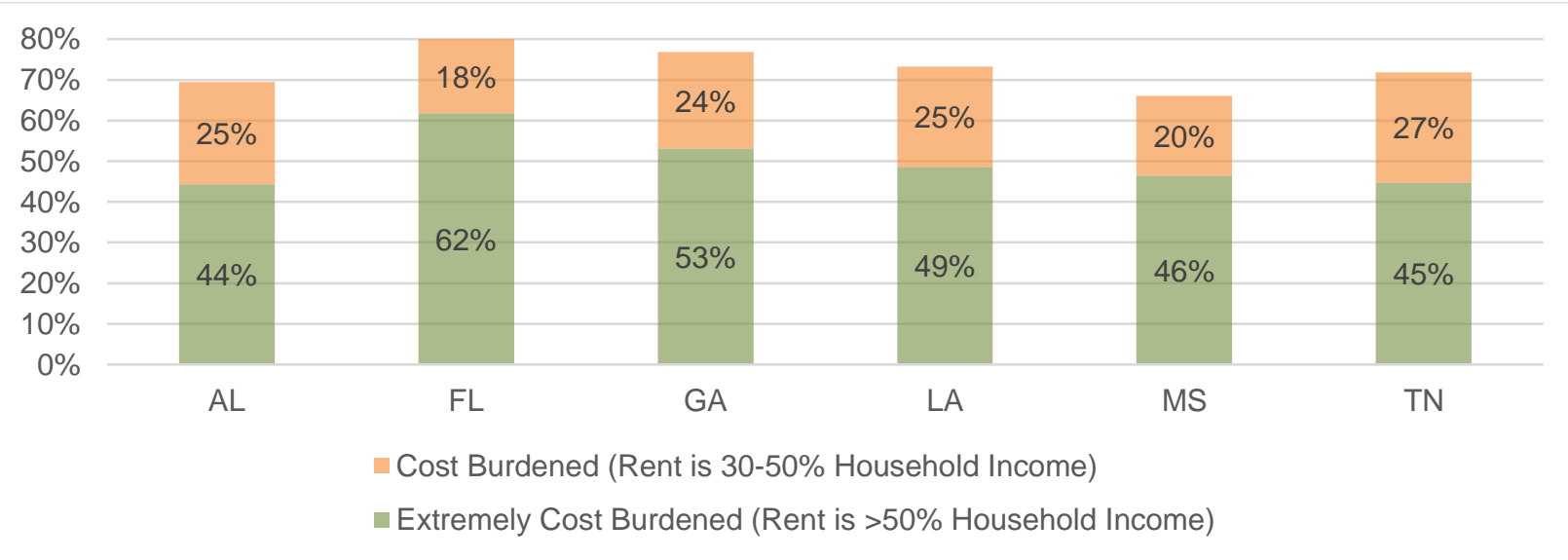

Source: Authors' tabulations of U.S. Census Bureau's 2015 American Community Survey public use microdata sample (PUMS) data 
As noted previously, the data methodology used allowed us to report not only the number of units affordable at various levels of income, but also the number of units that are available for households at these income levels, or not rented by a higher-income household. In the six states in the Atlanta Fed's District, there are a total of 2.5 million renter households earning 50 percent or below AMI (by MSA), and only 1.4 million units both affordable and available to those households, for an overall shortage of 1.2 million units for extremely low- and very low-income renter households. In our results, we present statistics normalized by population - the number of affordable and available units per 100 tenants as well as the total gap in affordable units by geography.

A perfectly balanced housing market would have around 100 affordable and available units per 100 tenants at each income level. However, given current economic conditions, significant gaps are common, particularly at lower levels of income. As shown in figure 7, Florida had the smallest number of units affordable and available per 100 renter households at all levels of income. Florida had a significant gap at or below 30 percent AMI (extremely low income), at or below 50 percent AMI (extremely low and very low income), and at or below 80 percent AMI (extremely low, very low, and low income). Alabama and Tennessee actually had a small surplus of units at 80 percent of AMI and below. Georgia, Louisiana, and Mississippi had similar levels of shortages at each level or income, with an even or nearly even supply of units at 80 percent of AMI and below. Clearly, the largest gaps are at 30 percent AMI and below in each state, with only about a fifth to a quarter of the units needed actually available.

\section{Figure 7: Affordable and Available Units per 100 Tenants by Income and State}

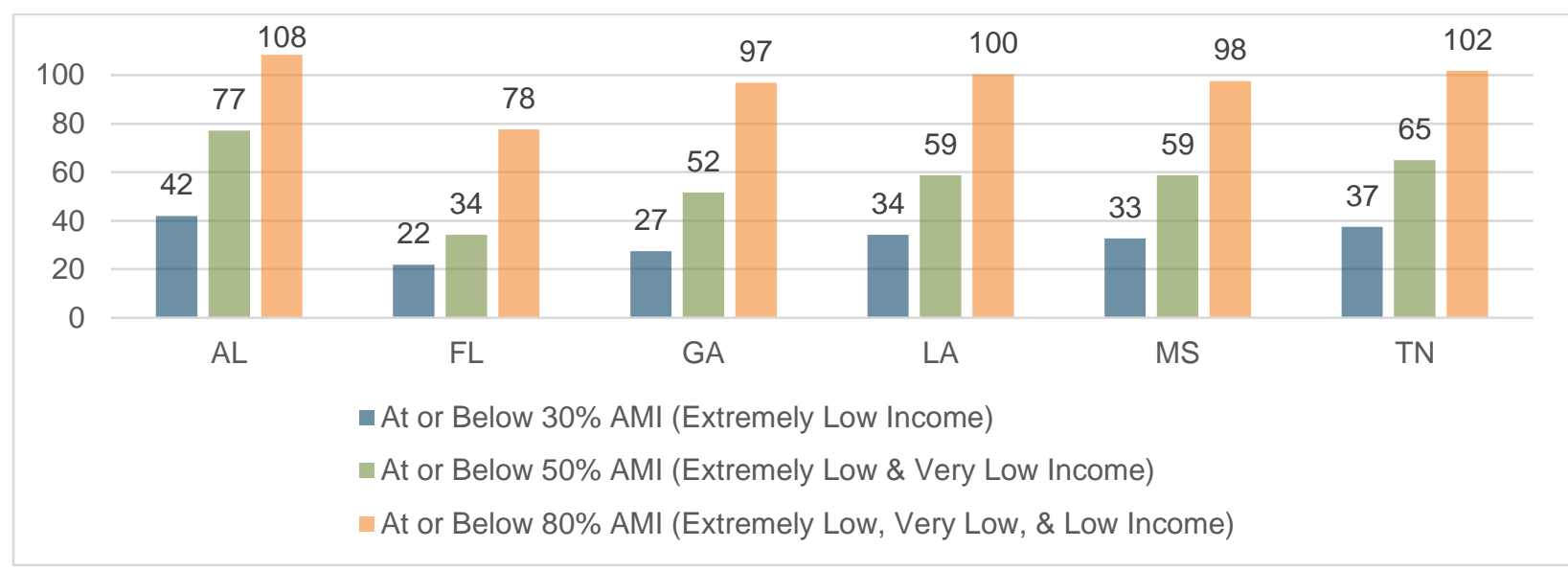

Source: Authors' tabulations of U.S. Census Bureau's 2015 American Community Survey public use microdata sample (PUMS) data

In absolute numbers, the surplus or deficit of affordable and available units varied quite a bit by state given differences in relative affordability and population size, with Florida experiencing the largest shortage of units at all three levels of income (see figure 8). The surpluses indicated above in Alabama and Tennessee at the 80 percent AMI and below level are relatively small compared to the large deficits found at other levels of income. However, the surpluses indicate a small cushion of affordable housing for the "workforce" segment of the market. Louisiana also has a much more modest surplus at 80 percent $\mathrm{AMI}$ and below. Of most concern are the hundreds of thousands of units needed to affordably house renters at 30 percent and 50 percent AMI and below in Florida, Georgia, Louisiana, and 
Tennessee and the tens of thousands of units needed in Alabama and Mississippi. Although all the southeastern states have the largest shortages per 100 units at 30 percent AMI and below, Alabama and Tennessee have the largest absolute unit shortages at the 50 percent AMI and below level. This may indicate that there are fewer extremely low-income households or a larger number of very low-income households in these states.

\section{Figure 8: Surplus or Deficit of Affordable and Available Units by Income and State}

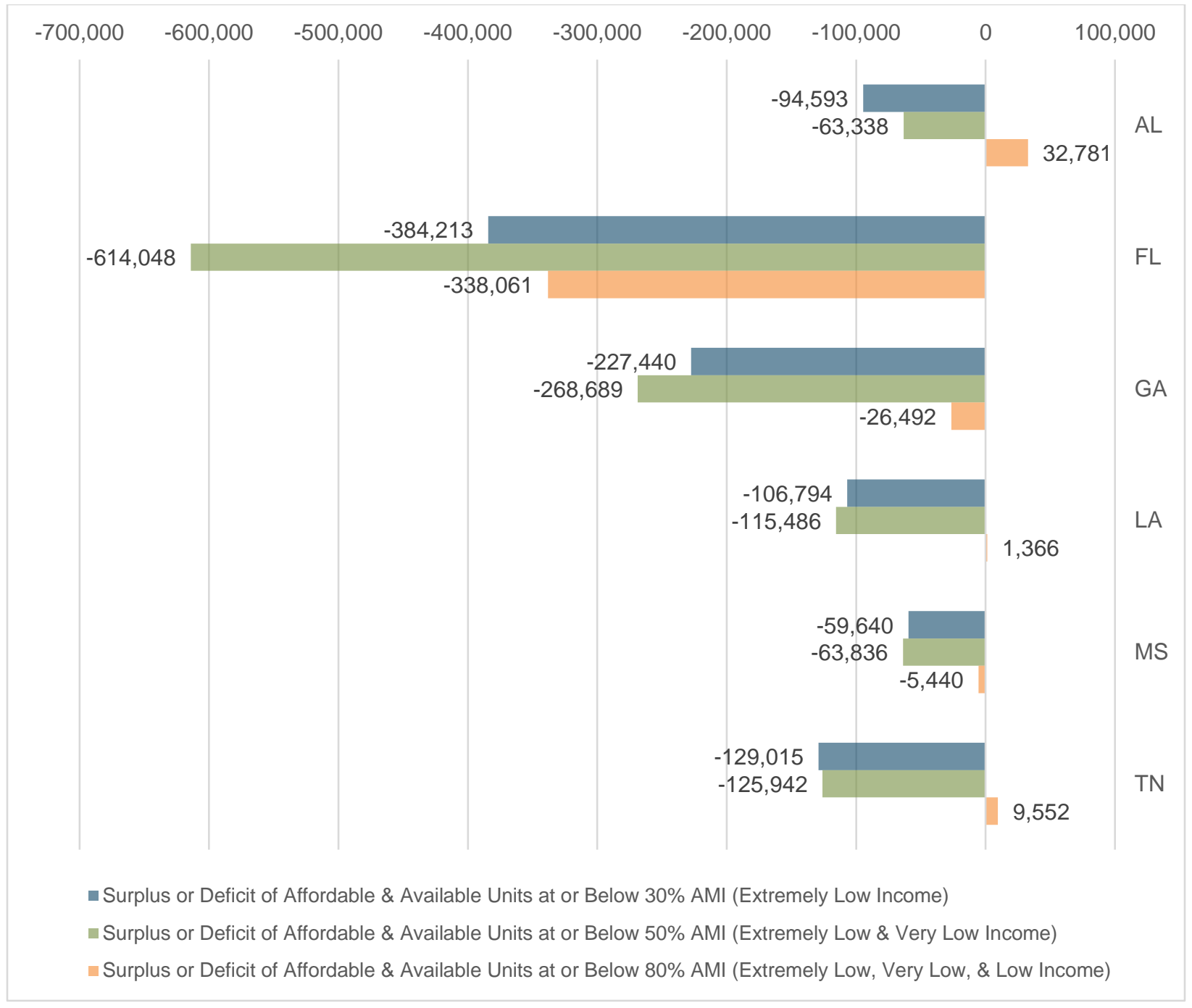

Source: Authors' tabulations of U.S. Census Bureau's 2015 American Community Survey public use microdata sample (PUMS) data

\section{Results: Combined PUMA-Level Regional Data}

Data were also calculated at the level of combined PUMA regions, which represent combinations of MSAs, micropolitan statistical areas, and in the case of the Miami MSA, larger cities within one MSA (Miami-Dade, Fort Lauderdale, and West Palm Beach-Boca Raton). The tables and charts below include only 25 out of 99 combined PUMA regions. As noted, the goal of this project was to 
provide detailed data for all metropolitan, micropolitan, and nonmetro areas in the Atlanta Fed's District. In the interest of space and readability, data for all 99 regions is in appendix B.

Table 3 shows the number and percent of cost-burdened renter households in the 25 combined PUMA regions with the largest MSA population in the Atlanta Fed's District. The names of each region are truncated for clarity from the full names found in appendices $A$ and B. Among the top 25 combined PUMA regions, the largest numbers of cost-burdened renter households are in Atlanta, Miami, Orlando, and Fort Lauderdale. The share of moderate- and upper-income households that are cost burdened is comparatively high in Florida metros, particularly Miami (66 percent of moderate-income households and 19 percent of upper-income households) and Fort Lauderdale (45 percent of moderate-income households and 14 percent of upper-income households).

Table 3: Number (and Percent) of Renter Households That Are Cost Burdened (Rent Is $>\mathbf{3 0}$ \% Household Income) by Income Category and by Combined PUMA Region (Top 25 MSAs by Population)

\begin{tabular}{|c|c|c|c|c|c|c|}
\hline Region (Alphabetically by State) & $\begin{array}{l}\text { Extremely } \\
\text { Low } \\
\text { Income } \\
\text { (30\% AMI or } \\
\text { less) }\end{array}$ & $\begin{array}{l}\text { Very Low } \\
\text { Income } \\
\text { (30.01 to } \\
50 \% \text { AMI) }\end{array}$ & $\begin{array}{l}\text { Low } \\
\text { Income } \\
\text { (50.01 to } \\
80 \% \text { AMI) }\end{array}$ & $\begin{array}{l}\text { Moderate } \\
\text { Income } \\
\text { (80.01 to } \\
120 \% \text { AMI) }\end{array}$ & $\begin{array}{l}\text { Upper } \\
\text { Income } \\
\text { (More than } \\
120 \% \text { AMI) }\end{array}$ & $\begin{array}{l}\text { All } \\
\text { Renter } \\
\text { House- } \\
\text { holds }\end{array}$ \\
\hline Birmingham-Hoover, AL & $\begin{array}{c}30,042 \\
(74 \%)\end{array}$ & $\begin{array}{c}18,766 \\
(73 \%)\end{array}$ & $\begin{array}{l}8,476 \\
(34 \%)\end{array}$ & $\begin{array}{c}1,253 \\
(6 \%)\end{array}$ & $\begin{array}{l}364 \\
(2 \%)\end{array}$ & $\begin{array}{c}58,901 \\
(43 \%)\end{array}$ \\
\hline Huntsville, AL & $\begin{array}{c}12,504 \\
(76 \%)\end{array}$ & $\begin{array}{l}9,782 \\
(67 \%)\end{array}$ & $\begin{array}{l}3,128 \\
(28 \%)\end{array}$ & $\begin{array}{l}463 \\
(4 \%)\end{array}$ & $\begin{array}{c}0 \\
(0 \%)\end{array}$ & $\begin{array}{l}25,877 \\
(41 \%)\end{array}$ \\
\hline Mobile, AL & $\begin{array}{l}9,905 \\
(79 \%)\end{array}$ & $\begin{array}{l}9,419 \\
(81 \%)\end{array}$ & $\begin{array}{l}4,529 \\
(40 \%)\end{array}$ & $\begin{array}{l}1,347 \\
(16 \%)\end{array}$ & $\begin{array}{c}64 \\
(1 \%)\end{array}$ & $\begin{array}{c}25,264 \\
(47 \%)\end{array}$ \\
\hline Cape Coral-Fort Myers, FL & $\begin{array}{l}10,526 \\
(71 \%)\end{array}$ & $\begin{array}{l}12,279 \\
(88 \%)\end{array}$ & $\begin{array}{c}11,052 \\
(66 \%)\end{array}$ & $\begin{array}{l}3,961 \\
(25 \%)\end{array}$ & $\begin{array}{c}1,472 \\
(8 \%)\end{array}$ & $\begin{array}{c}39,290 \\
(49 \%)\end{array}$ \\
\hline $\begin{array}{l}\text { Deltona-Daytona Beach-Ormond } \\
\text { Beach, FL }\end{array}$ & $\begin{array}{l}8,162 \\
(68 \%)\end{array}$ & $\begin{array}{l}9,430 \\
(80 \%)\end{array}$ & $\begin{array}{c}14,671 \\
(82 \%)\end{array}$ & $\begin{array}{l}5,077 \\
(38 \%)\end{array}$ & $\begin{array}{l}787 \\
(4 \%)\end{array}$ & $\begin{array}{c}38,127 \\
(52 \%)\end{array}$ \\
\hline Fort Lauderdale, FL & $\begin{array}{c}33,216 \\
(80 \%)\end{array}$ & $\begin{array}{c}38,276 \\
(93 \%)\end{array}$ & $\begin{array}{l}43,551 \\
(84 \%)\end{array}$ & $\begin{array}{c}24,871 \\
(45 \%)\end{array}$ & $\begin{array}{l}9,955 \\
(14 \%)\end{array}$ & $\begin{array}{c}149,869 \\
(58 \%)\end{array}$ \\
\hline Jacksonville, FL & $\begin{array}{l}33,637 \\
(75 \%)\end{array}$ & $\begin{array}{c}26,633 \\
(86 \%)\end{array}$ & $\begin{array}{l}25,756 \\
(56 \%)\end{array}$ & $\begin{array}{l}7,180 \\
(19 \%)\end{array}$ & $\begin{array}{c}2,658 \\
(6 \%)\end{array}$ & $\begin{array}{c}95,864 \\
(47 \%)\end{array}$ \\
\hline Lakeland, FL & $\begin{array}{l}9,205 \\
(75 \%) \\
\end{array}$ & $\begin{array}{l}9,306 \\
(84 \%)\end{array}$ & $\begin{array}{c}12,885 \\
(68 \%)\end{array}$ & $\begin{array}{l}5,385 \\
(37 \%)\end{array}$ & $\begin{array}{l}1,873 \\
(10 \%) \\
\end{array}$ & $\begin{array}{c}38,654 \\
(51 \%)\end{array}$ \\
\hline Miami-Dade, FL & $\begin{array}{c}54,666 \\
(66 \%)\end{array}$ & $\begin{array}{l}59,453 \\
(85 \%)\end{array}$ & $\begin{array}{c}70,684 \\
(85 \%)\end{array}$ & $\begin{array}{l}49,537 \\
(66 \%)\end{array}$ & $\begin{array}{c}23,950 \\
(19 \%)\end{array}$ & $\begin{array}{c}258,290 \\
(59 \%)\end{array}$ \\
\hline $\begin{array}{l}\text { North Port-Sarasota-Bradenton- } \\
\text { Venice, FL }\end{array}$ & $\begin{array}{l}9,796 \\
(75 \%)\end{array}$ & $\begin{array}{l}13,737 \\
(83 \%)\end{array}$ & $\begin{array}{c}12,981 \\
(62 \%)\end{array}$ & $\begin{array}{l}5,711 \\
(29 \%)\end{array}$ & $\begin{array}{c}1,758 \\
(8 \%)\end{array}$ & $\begin{array}{c}43,983 \\
(47 \%)\end{array}$ \\
\hline Orlando-Kissimmee-Sanford, FL & $\begin{array}{l}40,870 \\
(78 \%)\end{array}$ & $\begin{array}{l}57,637 \\
(94 \%)\end{array}$ & $\begin{array}{c}57,209 \\
(75 \%)\end{array}$ & $\begin{array}{c}19,248 \\
(29 \%)\end{array}$ & $\begin{array}{c}3,525 \\
(4 \%)\end{array}$ & $\begin{array}{c}178,489 \\
(52 \%)\end{array}$ \\
\hline
\end{tabular}




\begin{tabular}{|c|c|c|c|c|c|c|}
\hline Region (Alphabetically by State) & $\begin{array}{l}\text { Extremely } \\
\text { Low } \\
\text { Income } \\
\text { (30\% AMl or } \\
\text { less) }\end{array}$ & $\begin{array}{l}\text { Very Low } \\
\text { Income } \\
\text { (30.01 to } \\
50 \% \text { AMI) }\end{array}$ & $\begin{array}{l}\text { Low } \\
\text { Income } \\
\text { (50.01 to } \\
80 \% \text { AMI) }\end{array}$ & $\begin{array}{l}\text { Moderate } \\
\text { Income } \\
\text { (80.01 to } \\
120 \% \text { AMI) }\end{array}$ & $\begin{array}{c}\text { Upper } \\
\text { Income } \\
\text { (More than } \\
120 \% \text { AMI) }\end{array}$ & $\begin{array}{c}\text { All } \\
\text { Renter } \\
\text { House- } \\
\text { holds }\end{array}$ \\
\hline $\begin{array}{l}\text { Palm Bay-Melbourne-Titusville, } \\
\text { FL }\end{array}$ & $\begin{array}{l}7,840 \\
(69 \%)\end{array}$ & $\begin{array}{l}9,418 \\
(79 \%)\end{array}$ & $\begin{array}{l}8,496 \\
(59 \%)\end{array}$ & $\begin{array}{l}4,570 \\
(31 \%)\end{array}$ & $\begin{array}{l}762 \\
(5 \%)\end{array}$ & $\begin{array}{c}31,086 \\
(46 \%)\end{array}$ \\
\hline Pensacola-Ferry Pass-Brent, FL & $\begin{array}{l}7,197 \\
(70 \%)\end{array}$ & $\begin{array}{l}7,544 \\
(87 \%)\end{array}$ & $\begin{array}{l}7,984 \\
(57 \%)\end{array}$ & $\begin{array}{l}2,174 \\
(15 \%)\end{array}$ & $\begin{array}{l}531 \\
(3 \%)\end{array}$ & $\begin{array}{l}25,430 \\
(41 \%)\end{array}$ \\
\hline Port St. Lucie, FL & $\begin{array}{l}6,240 \\
(74 \%)\end{array}$ & $\begin{array}{l}7,459 \\
(90 \%)\end{array}$ & $\begin{array}{l}7,108 \\
(79 \%)\end{array}$ & $\begin{array}{l}2,412 \\
(27 \%)\end{array}$ & $\begin{array}{l}638 \\
(5 \%)\end{array}$ & $\begin{array}{c}23,857 \\
(49 \%)\end{array}$ \\
\hline $\begin{array}{l}\text { Tampa-St. Petersburg- } \\
\text { Clearwater, FL }\end{array}$ & $\begin{array}{c}57,863 \\
(73 \%)\end{array}$ & $\begin{array}{l}60,926 \\
(88 \%)\end{array}$ & $\begin{array}{c}57,022 \\
(66 \%)\end{array}$ & $\begin{array}{l}22,888 \\
(27 \%)\end{array}$ & $\begin{array}{l}6,223 \\
(5 \%)\end{array}$ & $\begin{array}{c}204,922 \\
(47 \%)\end{array}$ \\
\hline West Palm Beach-Boca Raton, FL & $\begin{array}{c}28,957 \\
(77 \%)\end{array}$ & $\begin{array}{l}25,966 \\
(88 \%)\end{array}$ & $\begin{array}{c}26,514 \\
(70 \%)\end{array}$ & $\begin{array}{l}11,270 \\
(37 \%)\end{array}$ & $\begin{array}{c}2,720 \\
(6 \%)\end{array}$ & $\begin{array}{c}95,427 \\
(53 \%)\end{array}$ \\
\hline $\begin{array}{l}\text { Atlanta-Sandy Springs-Roswell, } \\
\text { GA }\end{array}$ & $\begin{array}{c}128,776 \\
(77 \%)\end{array}$ & $\begin{array}{c}116,379 \\
(86 \%)\end{array}$ & $\begin{array}{l}81,643 \\
(51 \%)\end{array}$ & $\begin{array}{c}20,166 \\
(13 \%)\end{array}$ & $\begin{array}{c}4,165 \\
(2 \%)\end{array}$ & $\begin{array}{c}351,129 \\
(45 \%)\end{array}$ \\
\hline Baton Rouge, LA & $\begin{array}{l}18,954 \\
(73 \%)\end{array}$ & $\begin{array}{l}14,169 \\
(73 \%)\end{array}$ & $\begin{array}{l}7,224 \\
(40 \%)\end{array}$ & $\begin{array}{c}1,333 \\
(9 \%)\end{array}$ & $\begin{array}{l}531 \\
(4 \%)\end{array}$ & $\begin{array}{c}42,211 \\
(46 \%)\end{array}$ \\
\hline Lafayette, LA & $\begin{array}{l}11,943 \\
(70 \%)\end{array}$ & $\begin{array}{l}9,607 \\
(76 \%)\end{array}$ & $\begin{array}{l}2,742 \\
(26 \%)\end{array}$ & $\begin{array}{l}914 \\
(8 \%)\end{array}$ & $\begin{array}{c}80 \\
(1 \%)\end{array}$ & $\begin{array}{c}24,996 \\
(40 \%)\end{array}$ \\
\hline New Orleans-Metairie, LA & $\begin{array}{l}36,839 \\
(72 \%)\end{array}$ & $\begin{array}{l}27,994 \\
(87 \%)\end{array}$ & $\begin{array}{l}22,284 \\
(61 \%)\end{array}$ & $\begin{array}{l}7,802 \\
(25 \%)\end{array}$ & $\begin{array}{c}1,764 \\
(4 \%)\end{array}$ & $\begin{array}{l}96,683 \\
(50 \%)\end{array}$ \\
\hline Shreveport-Bossier City, LA & $\begin{array}{l}11,471 \\
(68 \%)\end{array}$ & $\begin{array}{l}8,679 \\
(83 \%)\end{array}$ & $\begin{array}{l}8,093 \\
(59 \%)\end{array}$ & $\begin{array}{l}1,779 \\
(18 \%)\end{array}$ & $\begin{array}{l}807 \\
(7 \%)\end{array}$ & $\begin{array}{c}30,829 \\
(49 \%)\end{array}$ \\
\hline Gulfport-Biloxi-Pascagoula, MS & $\begin{array}{l}8,636 \\
(64 \%)\end{array}$ & $\begin{array}{l}8,701 \\
(78 \%)\end{array}$ & $\begin{array}{l}5,641 \\
(54 \%)\end{array}$ & $\begin{array}{l}1,639 \\
(13 \%)\end{array}$ & $\begin{array}{c}699 \\
(4 \%)\end{array}$ & $\begin{array}{c}25,316 \\
(40 \%)\end{array}$ \\
\hline Jackson, MS & $\begin{array}{c}13,599 \\
(67 \%)\end{array}$ & $\begin{array}{l}9,331 \\
(66 \%)\end{array}$ & $\begin{array}{l}9,005 \\
(57 \%)\end{array}$ & $\begin{array}{l}1,847 \\
(17 \%)\end{array}$ & $\begin{array}{l}466 \\
(3 \%)\end{array}$ & $\begin{array}{c}34,248 \\
(45 \%)\end{array}$ \\
\hline Knoxville, TN & $\begin{array}{c}23,559 \\
(70 \%)\end{array}$ & $\begin{array}{l}15,220 \\
(66 \%)\end{array}$ & $\begin{array}{l}9,061 \\
(35 \%)\end{array}$ & $\begin{array}{l}834 \\
(5 \%)\end{array}$ & $\begin{array}{l}520 \\
(3 \%)\end{array}$ & $\begin{array}{c}49,194 \\
(41 \%)\end{array}$ \\
\hline $\begin{array}{l}\text { Nashville-Davidson-- } \\
\text { Murfreesboro--Franklin, TN }\end{array}$ & $\begin{array}{l}40,413 \\
(75 \%)\end{array}$ & $\begin{array}{c}33,923 \\
(77 \%)\end{array}$ & $\begin{array}{l}22,117 \\
(42 \%)\end{array}$ & $\begin{array}{l}7,098 \\
(14 \%)\end{array}$ & $\begin{array}{c}1,460 \\
(3 \%)\end{array}$ & $\begin{array}{c}105,011 \\
(42 \%)\end{array}$ \\
\hline
\end{tabular}

Source: Authors' tabulations of U.S. Census Bureau's 2015 American Community Survey public use microdata sample (PUMS) data

In terms of the percentage of cost-burdened renter households, the differences are not necessarily statistically different due to the relatively small populations within each income category in each MSA region. For all renter households, the percentage of cost-burdened households ranged from a low of 40 percent in both Lafayette, Louisiana, and Gulfport, Mississippi, to a high of 59 percent in Miami. Florida metros are among the most cost burdened overall and in each income category. Indeed, in many Florida metros-namely, Miami, Fort Lauderdale, Deltona, West Palm Beach, and Lakeland-a relatively large share of moderate-income renter households is cost burdened. Alarmingly, in Miami, one in three moderate-income households and one in five upper-income renter household are cost burdened. 
As shown in figure 9, the share of cost-burdened renter households with extremely low income and very low income (50 percent AMI and below) was also highest in many Florida combined PUMA regions. The total share of cost-burdened renter households at this income level ranged from a low of 67 percent in Jackson, Mississippi, to a high of 86 percent in Fort Lauderdale. Once again, differences between the percentages are not necessarily statistically significant.

\section{Figure 9: Percent of Extremely Low-Income and Very Low-Income Renter Households ( $<50 \%$ AMI) That Are Cost Burdened and Extremely Cost Burdened by Combined PUMA Region (Top 25 MSAs by Population)}

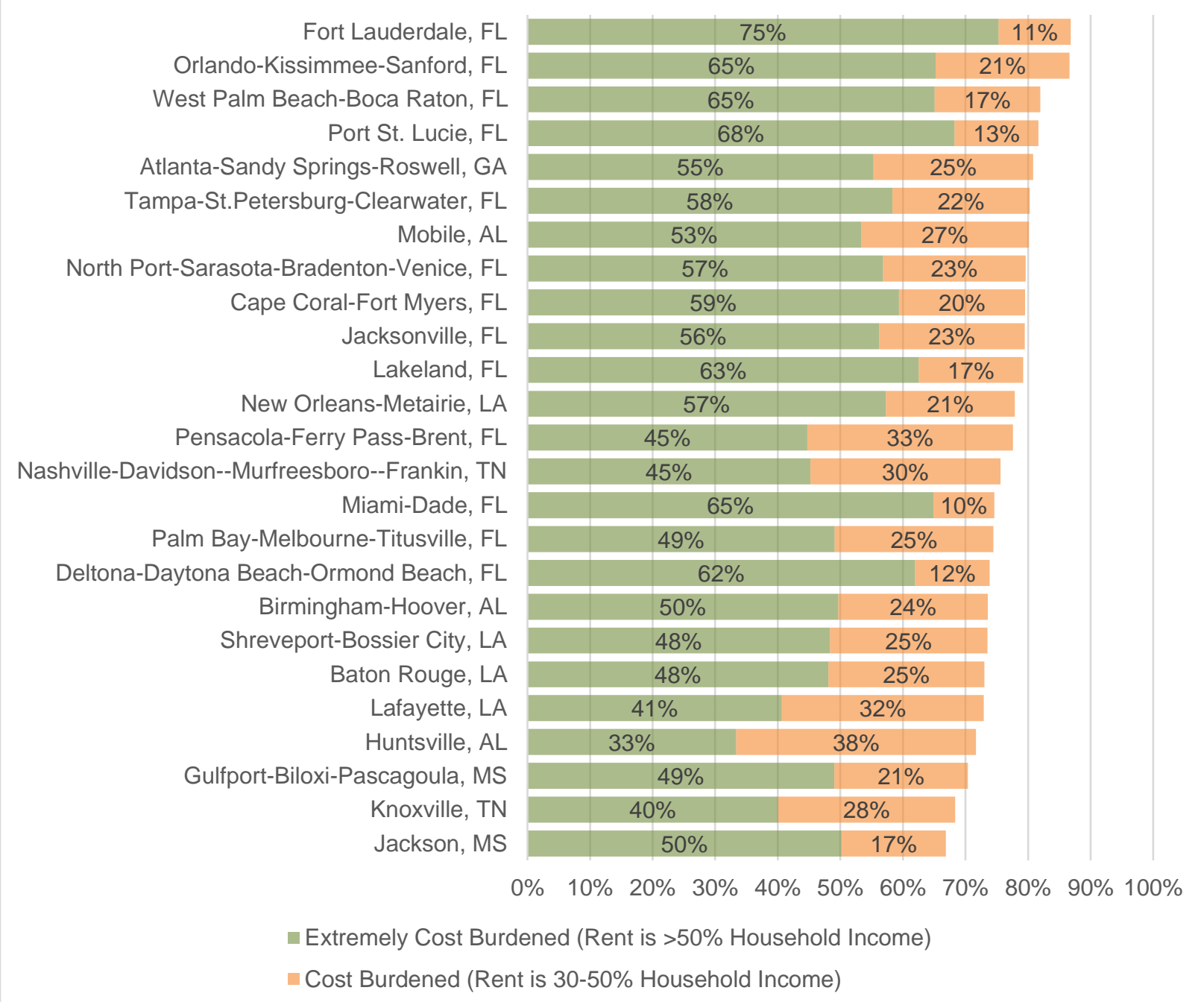

Source: Authors' tabulations of U.S. Census Bureau's 2015 American Community Survey public use microdata sample (PUMS) data 
Among combined PUMA regions in the Atlanta Fed's District, the number of affordable and available units per 100 tenants also varied by location and income level, with gaps found in all top-25 metros at both 50 percent $\mathrm{AMI}$ and 30 percent $\mathrm{AMI}$ and below (see table 4). At 80 percent $\mathrm{AMI}$ and below, nine metros had a small surplus, while Miami-Dade had a significant gap at only 41 units per 100 tenants. Units affordable and available per 100 renter households for households at 30 percent AMI and below (extremely low income) ranged from only 13 in Cape Coral-Fort Myers, Florida, to 50 in Huntsville, Alabama, indicating a significant gap in all MSAs at this level of income. High-cost markets such as Cape Coral-Fort Myers and Deltona-Daytona Beach-Ormond Beach, Florida, had notable gaps in affordable and available units for extremely low-income households.

We note this metric and the cost-burdened renter household metric are a function of the expense of rental housing in each market as well as of the overall supply of subsidized units relative to the number of households in need of assistance. Subsidized housing units include income limits and cap rental payments at an affordable level; therefore, by definition, they are affordable to extremely low-, very low-, and low-income households. Thus, markets with a more adequate supply of public housing, Section 8 , or other subsidized units to meet local demand will result in fewer cost-burdened households and more affordable and available units per 100 households at lower-income levels. In Miami-Dade, for example, there are 1.6 extremely low-income households per HUD-subsidized unit, while there are substantially more households in need per available unit in Fort Lauderdale (2.6) and Orlando (4.6). ${ }^{6}$

\footnotetext{
${ }^{6}$ Based on authors' tabulations of U.S. Census Bureau's 2015 American Community Survey PUMS data and HUD's 2015 Picture of Subsidized Households. Unit tabulations include public housing, Housing Choice Vouchers, ProjectBased Rental Assistance, and several smaller HUD programs (Section 8 Mod Rehab, Rent Sup and RAP, Section 236, Section 202/PRAC, and Section 811/PRAC).
} 
Table 4: Affordable and Available Units per 100 Tenants by AMI by Combined PUMA Region (Top 25 MSAs by Population)

\begin{tabular}{|c|c|c|c|}
\hline \multirow[b]{2}{*}{ Region (Alphabetically by State) } & \multicolumn{3}{|c|}{ Affordable and Available Units per 100 Tenants } \\
\hline & $\begin{array}{l}\text { At or Below 30\% AMI } \\
\text { (Extremely Low Income) }\end{array}$ & $\begin{array}{l}\text { At or Below 50\% AMI } \\
\text { (Extremely Low Income } \\
\text { and Very Low Income) }\end{array}$ & $\begin{array}{l}\text { At or Below 80\% AMI } \\
\text { (Very Low Income, } \\
\text { Extremely Low Income, } \\
\text { and Very Low Income) }\end{array}$ \\
\hline Birmingham-Hoover, AL & 36 & 73 & 105 \\
\hline Huntsville, AL & 50 & 92 & 111 \\
\hline Mobile, AL & 36 & 58 & 115 \\
\hline Cape Coral-Fort Myers, FL & 13 & 30 & 88 \\
\hline $\begin{array}{l}\text { Deltona-Daytona Beach- } \\
\text { Ormond Beach, FL }\end{array}$ & 14 & 28 & 69 \\
\hline Fort Lauderdale, FL & 15 & 17 & 50 \\
\hline Jacksonville, FL & 33 & 47 & 99 \\
\hline Lakeland, FL & 24 & 37 & 78 \\
\hline Miami-Dade, FL & 22 & 26 & 41 \\
\hline $\begin{array}{l}\text { North Port-Sarasota- } \\
\text { Bradenton-Venice, FL }\end{array}$ & 25 & 37 & 85 \\
\hline Orlando-Kissimmee-Sanford, FL & 14 & 24 & 79 \\
\hline $\begin{array}{l}\text { Palm Bay-Melbourne-Titusville, } \\
\text { FL }\end{array}$ & 26 & 52 & 106 \\
\hline Pensacola-Ferry Pass-Brent, FL & 36 & 73 & 115 \\
\hline Port St. Lucie, FL & 18 & 35 & 83 \\
\hline $\begin{array}{l}\text { Tampa-St. Petersburg- } \\
\text { Clearwater, FL }\end{array}$ & 23 & 33 & 88 \\
\hline $\begin{array}{l}\text { West Palm Beach-Boca Raton, } \\
\text { FL }\end{array}$ & 16 & 29 & 71 \\
\hline $\begin{array}{l}\text { Atlanta-Sandy Springs-Roswell, } \\
\text { GA }\end{array}$ & 24 & 47 & 98 \\
\hline Baton Rouge, LA & 31 & 70 & 105 \\
\hline Lafayette, LA & 46 & 71 & 104 \\
\hline New Orleans-Metairie, LA & 25 & 42 & 95 \\
\hline Shreveport-Bossier City, LA & 32 & 64 & 100 \\
\hline Gulfport-Biloxi-Pascagoula, MS & 30 & 56 & 107 \\
\hline Jackson, MS & 34 & 55 & 101 \\
\hline Knoxville, TN & 46 & 71 & 102 \\
\hline $\begin{array}{l}\text { Nashville-Davidson-- } \\
\text { Murfreesboro--Franklin, TN }\end{array}$ & 38 & 58 & 97 \\
\hline
\end{tabular}


Figure 10 shows affordable and available units at 50 percent AMI or below by combined PUMA region, sorted by greatest relative need. Among the 25 combined PUMA regions by MSA population, units at 50 percent $A M I$ and below (extremely low and very low income) range from 17 in Fort Lauderdale to 92 in Huntsville. The relatively small gap in Huntsville is likely due to the relatively high MSA AMI and a higher share of higher-income households.

\section{Figure 10: Affordable and Available Units per 100 Extremely Low- and Very Low-Income ( $<50 \%$ AMI) Tenants by Combined PUMA Region (Top 25 MSAs by Population)}

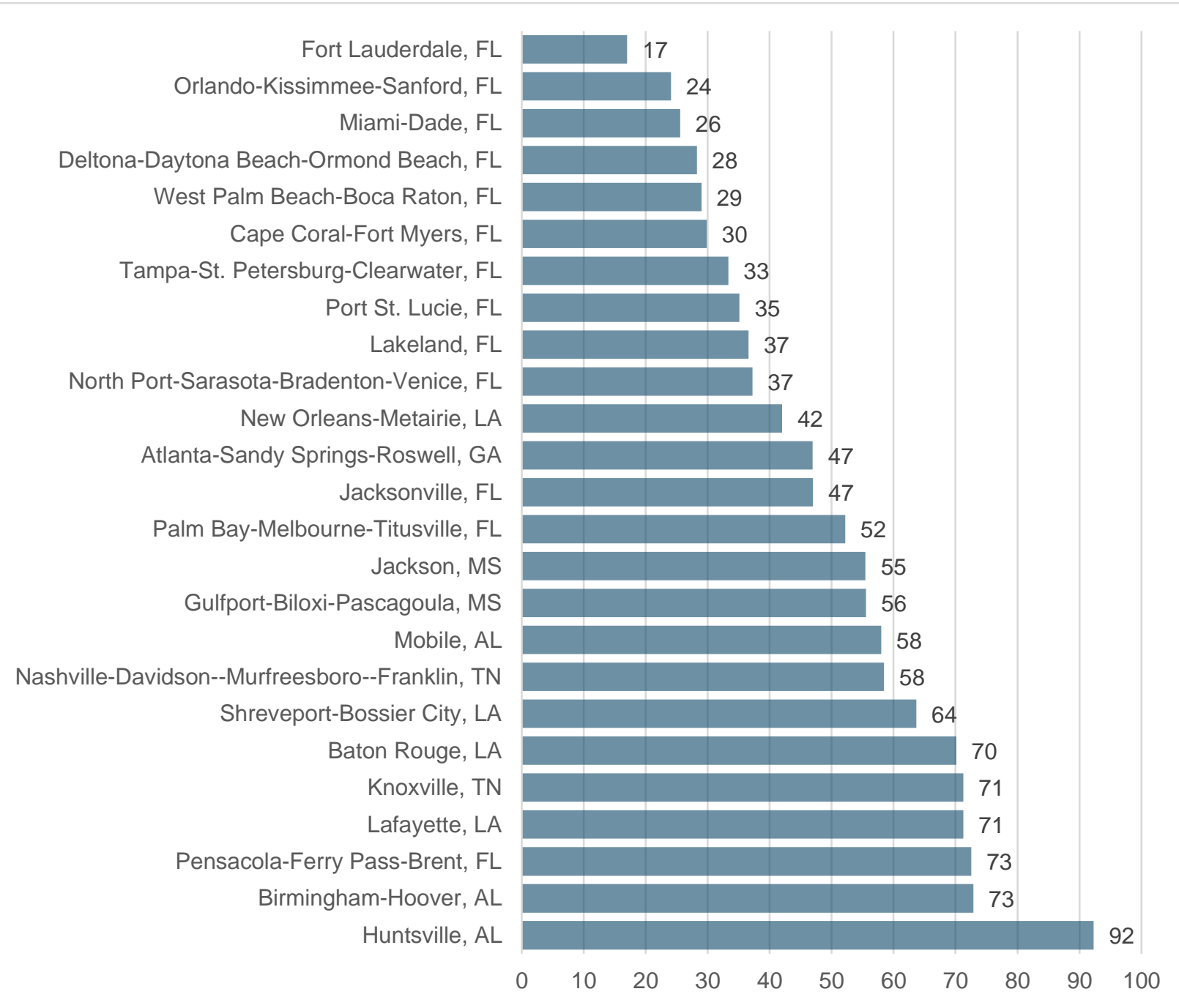

Source: Authors' tabulations of U.S. Census Bureau's 2015 American Community Survey public use microdata sample (PUMS) data

Among the 25 largest combined PUMA regions by MSA population, there is variation in the absolute surplus or deficit in units, which is also influenced by the populations of each respective PUMA region (see table 5). Although some regions have greater shortages at the level of 30 percent $\mathrm{AMI}$ and below (extremely low income), others have a greater shortage at the level of 50 percent AMI and below 
(extremely low income and very low income). Several metros had small surpluses at the level of 80 percent $\mathrm{AMI}$ and below.

Table 5: Surplus or Deficit of Affordable and Available Units by AMI by Combined PUMA Region (Top 25 MSAs by Population)

\begin{tabular}{|c|c|c|c|}
\hline \multirow[b]{2}{*}{ Region (Alphabetically by State) } & \multicolumn{3}{|c|}{ Surplus or Deficit of Affordable and Available Units } \\
\hline & $\begin{array}{l}\text { At or Below 30\% AMI } \\
\text { (Extremely Low Income) }\end{array}$ & $\begin{array}{l}\text { At or Below 50\% AMI } \\
\text { (Extremely Low Income } \\
\text { and Very Low Income) }\end{array}$ & $\begin{array}{l}\text { At or Below 80\% AMI } \\
\text { (Very Low Income, } \\
\text { Extremely Low Income, } \\
\text { and Very Low Income) }\end{array}$ \\
\hline Birmingham-Hoover, AL & $-25,895$ & $-17,985$ & 4,594 \\
\hline Huntsville, AL & $-8,234$ & $-2,400$ & 4,753 \\
\hline Mobile, AL & $-7,998$ & $-10,133$ & 5,371 \\
\hline Cape Coral-Fort Myers, FL & $-12,853$ & $-20,122$ & $-5,597$ \\
\hline $\begin{array}{l}\text { Deltona-Daytona Beach- } \\
\text { Ormond Beach, FL }\end{array}$ & $-10,403$ & $-17,081$ & $-13,003$ \\
\hline Fort Lauderdale, FL & $-34,974$ & $-68,662$ & $-67,165$ \\
\hline Jacksonville, FL & $-29,913$ & $-40,184$ & $-1,328$ \\
\hline Lakeland, FL & $-9,399$ & $-14,823$ & $-9,267$ \\
\hline Miami-Dade, FL & $-64,861$ & $-113,874$ & $-140,351$ \\
\hline $\begin{array}{l}\text { North Port-Sarasota- } \\
\text { Bradenton-Venice, FL }\end{array}$ & $-9,824$ & $-18,553$ & $-7,455$ \\
\hline Orlando-Kissimmee-Sanford, FL & $-45,055$ & $-86,356$ & $-39,466$ \\
\hline $\begin{array}{l}\text { Palm Bay-Melbourne-Titusville, } \\
\text { FL }\end{array}$ & $-8,326$ & $-11,080$ & 2,403 \\
\hline Pensacola-Ferry Pass-Brent, FL & $-6,627$ & $-5,216$ & 5,053 \\
\hline Port St. Lucie, FL & $-6,975$ & $-10,885$ & $-4,428$ \\
\hline $\begin{array}{l}\text { Tampa-St. Petersburg- } \\
\text { Clearwater, FL }\end{array}$ & $-60,783$ & $-98,686$ & $-28,022$ \\
\hline $\begin{array}{l}\text { West Palm Beach-Boca Raton, } \\
\text { FL }\end{array}$ & $-31,360$ & $-47,284$ & $-30,265$ \\
\hline $\begin{array}{l}\text { Atlanta-Sandy Springs-Roswell, } \\
\text { GA }\end{array}$ & $-127,604$ & $-160,953$ & $-10,695$ \\
\hline Baton Rouge, LA & $-18,063$ & $-13,550$ & 3,438 \\
\hline Lafayette, LA & $-9,103$ & $-8,494$ & 1,595 \\
\hline New Orleans-Metairie, LA & $-38,019$ & $-48,273$ & $-6,264$ \\
\hline Shreveport-Bossier City, LA & $-11,431$ & $-9,962$ & 72 \\
\hline Gulfport-Biloxi-Pascagoula, MS & $-9,348$ & $-10,952$ & 2,491 \\
\hline Jackson, MS & $-13,380$ & $-15,268$ & 307 \\
\hline
\end{tabular}




\begin{tabular}{|c|c|c|c|}
\hline & \multicolumn{3}{|c|}{ Surplus or Deficit of Affordable and Available Units } \\
\hline Region (Alphabetically by State) & $\begin{array}{l}\text { At or Below 30\% AMI } \\
\text { (Extremely Low Income) }\end{array}$ & $\begin{array}{l}\text { At or Below 50\% AMI } \\
\text { (Extremely Low Income } \\
\text { and Very Low Income) }\end{array}$ & $\begin{array}{l}\text { At or Below 80\% AMI } \\
\text { (Very Low Income, } \\
\text { Extremely Low Income, } \\
\text { and Very Low Income) }\end{array}$ \\
\hline Knoxville, TN & $-18,392$ & $-16,307$ & 1,628 \\
\hline $\begin{array}{l}\text { Nashville-Davidson-- } \\
\text { Murfreesboro--Franklin, TN }\end{array}$ & $-33,747$ & $-40,844$ & $-3,810$ \\
\hline
\end{tabular}

Source: Authors' tabulations of U.S. Census Bureau's 2015 American Community Survey public use microdata sample (PUMS) data

The shortage of affordable and available units at 50 percent AMI and below in absolute numbers is shown in figure 11 , sorted by combined PUMA regions with the greatest to least need. The deficit ranged from only 2,400 units in Huntsville to 160,953 units in Atlanta-Sandy Springs-Roswell. These numbers are a reasonable approximation; however, we again note that the PUMA boundaries are not an exact fit to MSAs. Therefore, the calculations may contain areas outside of or exclude areas in the MSA limits. Given their populations, large metros such as Atlanta, Miami, Tampa, Orlando, New Orleans, and Nashville had among the largest deficits in the Southeast for extremely low- and very low-income levels. As noted previously, we split the Miami MSA into smaller regions for analysis (Miami-Dade, Fort Lauderdale, and West Palm Beach-Boca Raton). Combining these areas together would amount to a shortage of 229,820 units at this income level for the larger Miami-Fort Lauderdale-West Palm Beach MSA region. 
Figure 11: Deficit of Affordable and Available Units for Extremely Low- and Very LowIncome ( $<50 \%$ AMI) Households by Combined PUMA Region (Top 25 MSAs by Population)

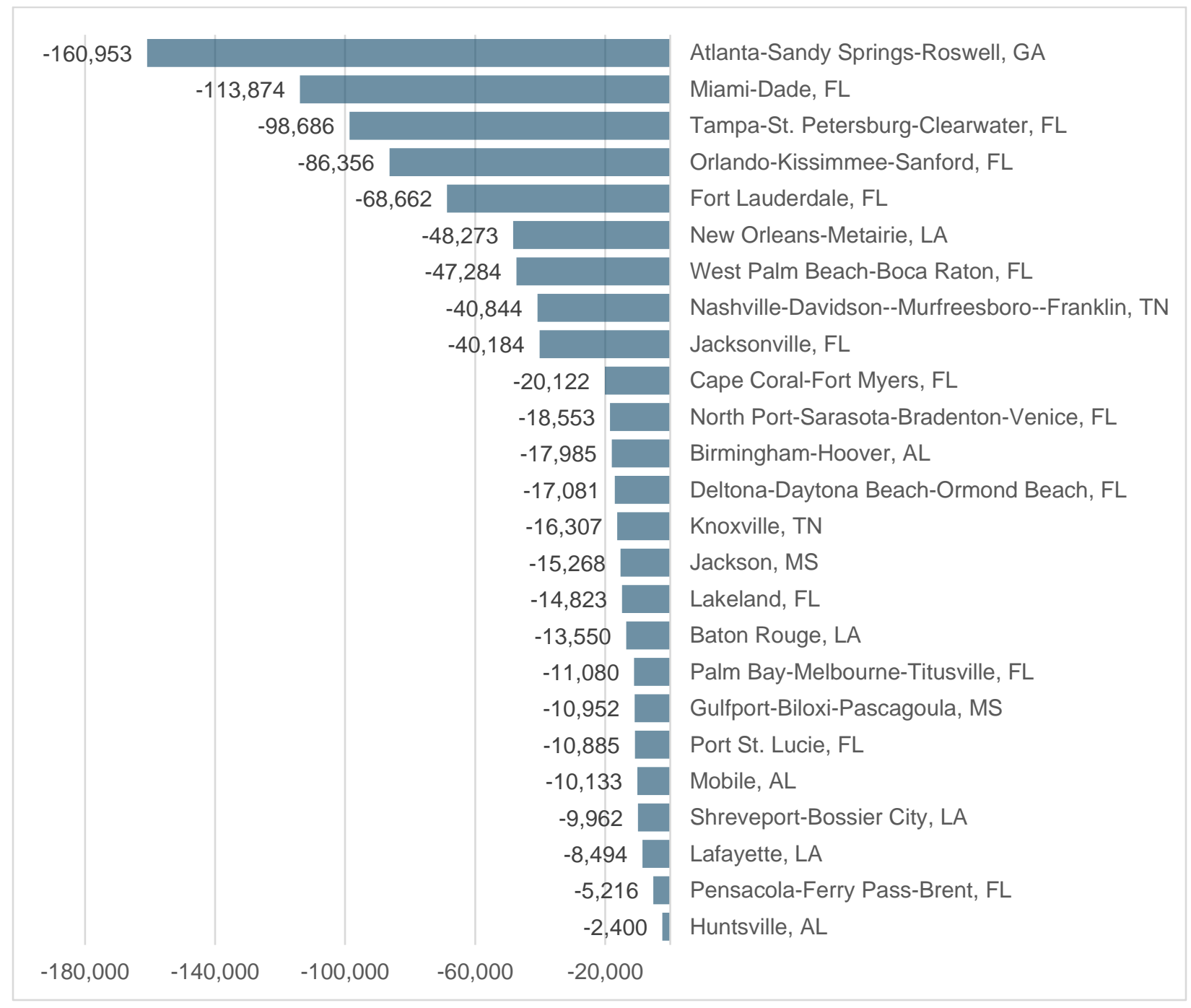

Source: Authors' tabulations of U.S. Census Bureau's 2015 American Community Survey public use microdata sample (PUMS) data

\section{Results: City-Level Data}

We also provide city-level data, as many housing programs and policies are administered at the local level. As shown in table 6, the number of cost-burdened households was largest in Miami, Jacksonville, and Memphis. Although the percentage differences are not necessarily statistically significant, the percent of cost-burdened renter households ranges from a low of 42 percent in Knoxville, Tennessee, to a high of 64 percent in Hialeah, Florida. Many Florida cities are among the most cost burdened overall and in each income category, including moderate and upper income. We note that cities, like MSAs, do not always fit cleanly in PUMAs, therefore, we chose only those cities with a reasonably close fit or combined city jurisdictions. Fort Lauderdale was combined with Pompano Beach, 
Tamarac, and Oakland Park due to the number of overlapping PUMAs in that area. For the citywide calculations, these estimates may differ from other sources for this reason.

Table 6: Number (and Percent) of Renter Households That Are Cost Burdened (Rent Is $>\mathbf{3 0} \%$ Household Income) by Income Category and by City

\begin{tabular}{|c|c|c|c|c|c|c|}
\hline City (Alphabetically by State) & $\begin{array}{c}\text { Extremely } \\
\text { Low } \\
\text { Income } \\
\text { (30\% AMl or } \\
\text { less) }\end{array}$ & $\begin{array}{c}\text { Very } \\
\text { Low } \\
\text { Income } \\
\text { (30.01 to } \\
50 \% \text { AMI) }\end{array}$ & $\begin{array}{l}\text { Low } \\
\text { Income } \\
\text { (50.01 to } \\
80 \% \text { AMI) }\end{array}$ & $\begin{array}{l}\text { Moderate } \\
\text { Income } \\
\text { (80.01 to } \\
120 \% \text { AMI) }\end{array}$ & $\begin{array}{l}\text { Upper } \\
\text { Income } \\
\text { (More than } \\
120 \% \text { AMI) }\end{array}$ & $\begin{array}{c}\text { All } \\
\text { Renter } \\
\text { House- } \\
\text { holds }\end{array}$ \\
\hline Birmingham, AL & $\begin{array}{c}14,075 \\
(76 \%)\end{array}$ & $\begin{array}{l}6,446 \\
(60 \%)\end{array}$ & $\begin{array}{l}1,360 \\
(29 \%)\end{array}$ & $\begin{array}{l}338 \\
(5 \%)\end{array}$ & $\begin{array}{c}0 \\
(0 \%)\end{array}$ & $\begin{array}{c}22,219 \\
(48 \%)\end{array}$ \\
\hline Huntsville, AL & $\begin{array}{l}8,234 \\
(80 \%)\end{array}$ & $\begin{array}{l}7,217 \\
(69 \%)\end{array}$ & $\begin{array}{l}1,055 \\
(27 \%)\end{array}$ & $\begin{array}{l}170 \\
(3 \%)\end{array}$ & $\begin{array}{c}0 \\
(0 \%)\end{array}$ & $\begin{array}{l}16,676 \\
(43 \%)\end{array}$ \\
\hline Mobile, AL & $\begin{array}{l}8,113 \\
(77 \%)\end{array}$ & $\begin{array}{l}7,523 \\
(76 \%)\end{array}$ & $\begin{array}{l}1,988 \\
(34 \%)\end{array}$ & $\begin{array}{c}593 \\
(12 \%)\end{array}$ & $\begin{array}{c}64 \\
(1 \%)\end{array}$ & $\begin{array}{l}18,281 \\
(48 \%)\end{array}$ \\
\hline Montgomery, AL & $\begin{array}{l}7,267 \\
(70 \%)\end{array}$ & $\begin{array}{l}8,012 \\
(84 \%)\end{array}$ & $\begin{array}{l}1,478 \\
(35 \%)\end{array}$ & $\begin{array}{c}932 \\
(14 \%)\end{array}$ & $\begin{array}{c}15 \\
(0 \%)\end{array}$ & $\begin{array}{c}17,704 \\
(49 \%)\end{array}$ \\
\hline Tuscaloosa, AL & $\begin{array}{l}3,456 \\
(75 \%)\end{array}$ & $\begin{array}{l}4,330 \\
(74 \%)\end{array}$ & $\begin{array}{l}1,326 \\
(58 \%)\end{array}$ & $\begin{array}{c}838 \\
(22 \%)\end{array}$ & $\begin{array}{c}96 \\
(2 \%)\end{array}$ & $\begin{array}{l}10,046 \\
(47 \%)\end{array}$ \\
\hline Cape Coral, FL & $\begin{array}{l}2,064 \\
(85 \%)\end{array}$ & $\begin{array}{l}3,900 \\
(94 \%)\end{array}$ & $\begin{array}{l}1,785 \\
(74 \%)\end{array}$ & $\begin{array}{l}1,551 \\
(31 \%)\end{array}$ & $\begin{array}{c}0 \\
(0 \%)\end{array}$ & $\begin{array}{l}9,300 \\
(46 \%)\end{array}$ \\
\hline Fort Lauderdale, FL* & $\begin{array}{c}10,616 \\
(82 \%)\end{array}$ & $\begin{array}{c}16,451 \\
(94 \%)\end{array}$ & $\begin{array}{l}7,408 \\
(67 \%)\end{array}$ & $\begin{array}{l}6,069 \\
(39 \%)\end{array}$ & $\begin{array}{l}2,886 \\
(14 \%)\end{array}$ & $\begin{array}{l}43,430 \\
(55 \%)\end{array}$ \\
\hline Gainesville, FL & $\begin{array}{l}7,738 \\
(68 \%)\end{array}$ & $\begin{array}{l}6,375 \\
(80 \%)\end{array}$ & $\begin{array}{l}1,236 \\
(44 \%)\end{array}$ & $\begin{array}{c}791 \\
(15 \%)\end{array}$ & $\begin{array}{l}263 \\
(6 \%)\end{array}$ & $\begin{array}{l}16,403 \\
(51 \%)\end{array}$ \\
\hline Hialeah, FL & $\begin{array}{l}5,277 \\
(68 \%)\end{array}$ & $\begin{array}{l}8,376 \\
(85 \%)\end{array}$ & $\begin{array}{l}4,237 \\
(94 \%)\end{array}$ & $\begin{array}{l}2,999 \\
(61 \%)\end{array}$ & $\begin{array}{l}266 \\
(5 \%)\end{array}$ & $\begin{array}{l}21,155 \\
(64 \%)\end{array}$ \\
\hline Jacksonville, FL & $\begin{array}{c}25,431 \\
(78 \%)\end{array}$ & $\begin{array}{c}29,947 \\
(84 \%)\end{array}$ & $\begin{array}{c}10,773 \\
(49 \%)\end{array}$ & $\begin{array}{l}5,380 \\
(22 \%)\end{array}$ & $\begin{array}{l}978 \\
(3 \%)\end{array}$ & $\begin{array}{c}72,509 \\
(49 \%)\end{array}$ \\
\hline Miami, FL & $\begin{array}{l}18,250 \\
(65 \%)\end{array}$ & $\begin{array}{c}26,031 \\
(81 \%)\end{array}$ & $\begin{array}{c}11,084 \\
(78 \%)\end{array}$ & $\begin{array}{l}11,035 \\
(60 \%)\end{array}$ & $\begin{array}{l}7,989 \\
(28 \%)\end{array}$ & $\begin{array}{c}74,389 \\
(61 \%)\end{array}$ \\
\hline Orlando, FL & $\begin{array}{c}12,666 \\
(77 \%)\end{array}$ & $\begin{array}{c}28,350 \\
(89 \%)\end{array}$ & $\begin{array}{l}9,655 \\
(59 \%)\end{array}$ & $\begin{array}{l}5,281 \\
(24 \%)\end{array}$ & $\begin{array}{c}1,349 \\
(5 \%)\end{array}$ & $\begin{array}{c}57,301 \\
(52 \%)\end{array}$ \\
\hline Port St Lucie, FL & $\begin{array}{l}1,214 \\
(62 \%)\end{array}$ & $\begin{array}{c}2,595 \\
(100 \%)\end{array}$ & $\begin{array}{l}1,601 \\
(85 \%)\end{array}$ & $\begin{array}{c}887 \\
(26 \%)\end{array}$ & $\begin{array}{l}422 \\
(8 \%)\end{array}$ & $\begin{array}{l}6,719 \\
(45 \%)\end{array}$ \\
\hline St Petersburg, FL & $\begin{array}{l}6,280 \\
(68 \%)\end{array}$ & $\begin{array}{l}8,105 \\
(84 \%)\end{array}$ & $\begin{array}{l}3,923 \\
(61 \%)\end{array}$ & $\begin{array}{l}1,529 \\
(18 \%)\end{array}$ & $\begin{array}{c}900 \\
(8 \%)\end{array}$ & $\begin{array}{c}20,737 \\
(46 \%)\end{array}$ \\
\hline Tallahassee, FL & $\begin{array}{c}12,526 \\
(89 \%)\end{array}$ & $\begin{array}{c}10,096 \\
(84 \%)\end{array}$ & $\begin{array}{l}1,684 \\
(44 \%)\end{array}$ & $\begin{array}{l}547 \\
(9 \%)\end{array}$ & $\begin{array}{c}0 \\
(0 \%)\end{array}$ & $\begin{array}{c}24,853 \\
(63 \%)\end{array}$ \\
\hline Tampa, FL & $\begin{array}{c}19,875 \\
(73 \%)\end{array}$ & $\begin{array}{c}22,074 \\
(87 \%)\end{array}$ & $\begin{array}{l}6,259 \\
(56 \%)\end{array}$ & $\begin{array}{l}4,621 \\
(30 \%)\end{array}$ & $\begin{array}{c}1,430 \\
(6 \%)\end{array}$ & $\begin{array}{c}54,259 \\
(52 \%)\end{array}$ \\
\hline Athens-Clarke County, GA & $\begin{array}{l}7,215 \\
(74 \%)\end{array}$ & $\begin{array}{l}6,164 \\
(91 \%)\end{array}$ & $\begin{array}{l}1,619 \\
(60 \%)\end{array}$ & $\begin{array}{l}1,220 \\
(26 \%)\end{array}$ & $\begin{array}{c}76 \\
(2 \%)\end{array}$ & $\begin{array}{c}16,294 \\
(56 \%)\end{array}$ \\
\hline
\end{tabular}




\begin{tabular}{|c|c|c|c|c|c|c|}
\hline City (Alphabetically by State) & $\begin{array}{c}\text { Extremely } \\
\text { Low } \\
\text { Income } \\
\text { (30\% AMl or } \\
\text { less) }\end{array}$ & $\begin{array}{c}\text { Very } \\
\text { Low } \\
\text { Income } \\
\text { (30.01 to } \\
50 \% \text { AMI) }\end{array}$ & $\begin{array}{l}\text { Low } \\
\text { Income } \\
\text { (50.01 to } \\
80 \% \text { AMI) }\end{array}$ & $\begin{array}{l}\text { Moderate } \\
\text { Income } \\
\text { (80.01 to } \\
120 \% \text { AMI) }\end{array}$ & $\begin{array}{l}\text { Upper } \\
\text { Income } \\
\text { (More than } \\
120 \% \text { AMI) }\end{array}$ & $\begin{array}{c}\text { All } \\
\text { Renter } \\
\text { House- } \\
\text { holds }\end{array}$ \\
\hline Atlanta, GA & $\begin{array}{c}24,988 \\
(69 \%)\end{array}$ & $\begin{array}{c}21,382 \\
(80 \%)\end{array}$ & $\begin{array}{l}6,906 \\
(44 \%)\end{array}$ & $\begin{array}{l}4,853 \\
(21 \%)\end{array}$ & $\begin{array}{c}1,081 \\
(3 \%)\end{array}$ & $\begin{array}{c}59,210 \\
(44 \%)\end{array}$ \\
\hline Augusta-Richmond County, GA & $\begin{array}{l}8,287 \\
(81 \%)\end{array}$ & $\begin{array}{l}5,393 \\
(85 \%)\end{array}$ & $\begin{array}{l}1,931 \\
(56 \%)\end{array}$ & $\begin{array}{c}278 \\
(5 \%)\end{array}$ & $\begin{array}{c}89 \\
(1 \%)\end{array}$ & $\begin{array}{c}15,978 \\
(51 \%)\end{array}$ \\
\hline Columbus, GA & $\begin{array}{l}6,162 \\
(80 \%)\end{array}$ & $\begin{array}{l}6,902 \\
(74 \%)\end{array}$ & $\begin{array}{l}3,182 \\
(71 \%)\end{array}$ & $\begin{array}{l}2,405 \\
(28 \%)\end{array}$ & $\begin{array}{l}657 \\
(6 \%)\end{array}$ & $\begin{array}{c}19,308 \\
(47 \%)\end{array}$ \\
\hline Macon-Bibb County, GA & $\begin{array}{l}6,093 \\
(63 \%)\end{array}$ & $\begin{array}{l}5,535 \\
(82 \%)\end{array}$ & $\begin{array}{l}1,706 \\
(63 \%)\end{array}$ & $\begin{array}{c}489 \\
(14 \%)\end{array}$ & $\begin{array}{c}83 \\
(2 \%)\end{array}$ & $\begin{array}{c}13,906 \\
(51 \%)\end{array}$ \\
\hline Savannah, GA & $\begin{array}{l}5,921 \\
(73 \%)\end{array}$ & $\begin{array}{l}7,916 \\
(83 \%)\end{array}$ & $\begin{array}{l}3,007 \\
(68 \%)\end{array}$ & $\begin{array}{l}1,589 \\
(28 \%)\end{array}$ & $\begin{array}{l}215 \\
(3 \%)\end{array}$ & $\begin{array}{c}18,648 \\
(54 \%)\end{array}$ \\
\hline Baton Rouge, LA & $\begin{array}{l}7,690 \\
(81 \%) \\
\end{array}$ & $\begin{array}{l}6,300 \\
(59 \%) \\
\end{array}$ & $\begin{array}{l}1,078 \\
(30 \%)\end{array}$ & $\begin{array}{c}238 \\
(5 \%)\end{array}$ & $\begin{array}{c}531 \\
(11 \%)\end{array}$ & $\begin{array}{c}15,837 \\
(47 \%)\end{array}$ \\
\hline New Orleans, LA & $\begin{array}{c}20,244 \\
(74 \%)\end{array}$ & $\begin{array}{c}15,259 \\
(79 \%)\end{array}$ & $\begin{array}{l}4,580 \\
(61 \%)\end{array}$ & $\begin{array}{l}4,305 \\
(37 \%)\end{array}$ & $\begin{array}{c}1,109 \\
(6 \%)\end{array}$ & $\begin{array}{r}45,497 \\
(55 \%)\end{array}$ \\
\hline Jackson, MS & $\begin{array}{l}5,865 \\
(77 \%)\end{array}$ & $\begin{array}{l}6,342 \\
(79 \%)\end{array}$ & $\begin{array}{c}556 \\
(35 \%)\end{array}$ & $\begin{array}{l}195 \\
(7 \%)\end{array}$ & $\begin{array}{c}56 \\
(2 \%)\end{array}$ & $\begin{array}{c}13,014 \\
(55 \%)\end{array}$ \\
\hline Chattanooga, TN & $\begin{array}{l}8,306 \\
(81 \%)\end{array}$ & $\begin{array}{l}6,006 \\
(66 \%)\end{array}$ & $\begin{array}{l}5,199 \\
(49 \%)\end{array}$ & $\begin{array}{l}537 \\
(7 \%)\end{array}$ & $\begin{array}{l}105 \\
(2 \%)\end{array}$ & $\begin{array}{c}20,153 \\
(46 \%)\end{array}$ \\
\hline Knoxville, TN & $\begin{array}{l}8,034 \\
(65 \%)\end{array}$ & $\begin{array}{l}5,128 \\
(69 \%)\end{array}$ & $\begin{array}{l}3,344 \\
(35 \%)\end{array}$ & $\begin{array}{c}295 \\
(5 \%)\end{array}$ & $\begin{array}{c}301 \\
(5 \%)\end{array}$ & $\begin{array}{c}17,102 \\
(42 \%)\end{array}$ \\
\hline Memphis, TN & $\begin{array}{c}25,875 \\
(73 \%) \\
\end{array}$ & $\begin{array}{c}20,292 \\
(82 \%) \\
\end{array}$ & $\begin{array}{c}12,465 \\
(48 \%)\end{array}$ & $\begin{array}{l}2,206 \\
(14 \%)\end{array}$ & $\begin{array}{c}227 \\
(1 \%)\end{array}$ & $\begin{array}{c}61,065 \\
(51 \%)\end{array}$ \\
\hline Nashville-Davidson, TN & $\begin{array}{c}18,294 \\
(73 \%)\end{array}$ & $\begin{array}{c}17,635 \\
(82 \%)\end{array}$ & $\begin{array}{c}12,420 \\
(47 \%)\end{array}$ & $\begin{array}{l}3,269 \\
(13 \%)\end{array}$ & $\begin{array}{c}965 \\
(4 \%)\end{array}$ & $\begin{array}{c}52,583 \\
(43 \%)\end{array}$ \\
\hline
\end{tabular}

Source: Authors' tabulations of U.S. Census Bureau 2015 American Community Survey public use microdata sample (PUMS) data *Fort Lauderdale, Florida, area also includes Pompano Beach, Tamarac, and Oakland Park.

As with the combined PUMA data, differences in the percentage of cost-burdened renter households may not be statistically significant due to the small sample sizes within each income segment at the city level. As shown in figure 12, among extremely low-income and very low-income renter households, the share of cost-burdened renter households was highest in many Florida cities, namely, Cape Coral, Fort Lauderdale, Tallahassee, and Orlando. The total share of cost-burdened renter households with incomes of 50 percent of AMI or lower ranged from a low of 66 percent in Knoxville, Tennessee, to a high of 91 percent in Cape Coral, Florida. The percent of extremely cost-burdened renter households at this income level was particularly high in many Florida markets. 


\section{Figure 12: Percent Extremely Low- and Very Low-Income Renter Households ( $<50 \%$ AMI) That Are Cost Burdened and Extremely Cost Burdened by City}

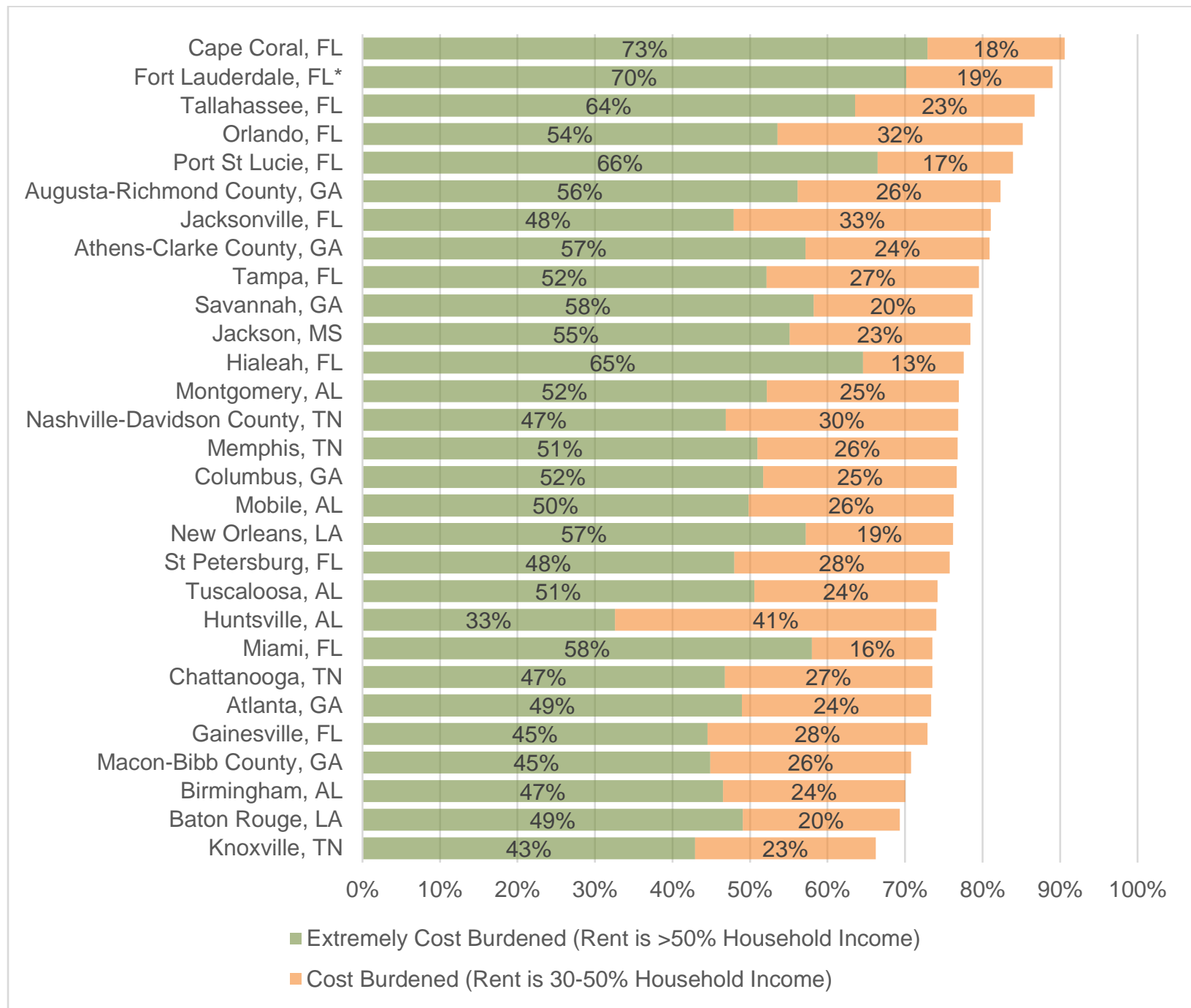

Source: Authors' tabulations of U.S. Census Bureau's 2015 American Community Survey public use microdata sample (PUMS) data *Fort Lauderdale, Florida, area also includes Pompano Beach, Tamarac, and Oakland Park.

At the city level, there is wide variation in the number of affordable and available units per 100 tenants at each income level, with an adequate number of units or surplus of workforce units only available at or below 80 percent AMI in 14 of 29 cities. At lower thresholds of income and in all other cities at 80 percent AMI or below, the number of affordable and available units per 100 tenants fell short of 100 (see table 7). As with the combined PUMA regions found in the previous section, the overall supply of subsidized units relative to the number of households in need of assistance also affects the number of cost-burdened households and the number of affordable and available units for low-income households at the city level. In Miami, for example, there are 1.7 extremely low-income households per 
Atlanta Fed Community \& Economic Development Discussion Paper Series • No. 02-18

HUD-subsidized unit, while there are substantially more households in need per available unit in Fort Lauderdale and its environs (2.5) and Orlando (3.9). ${ }^{7}$

7 Based on authors' tabulations of U.S. Census Bureau's 2015 American Community Survey PUMS data and HUD's 2015 Picture of Subsidized Households. Unit tabulations include public housing, Housing Choice Vouchers, ProjectBased Rental Assistance, and several smaller HUD programs (Section 8 Mod Rehab, Rent Sup and RAP, Section 236, Section 202/PRAC, and Section 811/PRAC). 


\section{Table 7: Affordable and Available Units per 100 Tenants by AMI by City}

\begin{tabular}{|c|c|c|c|}
\hline \multirow[b]{2}{*}{ City (Alphabetically by State) } & \multicolumn{3}{|c|}{ Affordable and Available Units per 100 Tenants } \\
\hline & $\begin{array}{l}\text { At or Below 30\% AMI } \\
\text { (Extremely Low Income) }\end{array}$ & $\begin{array}{l}\text { At or Below 50\% AMI } \\
\text { (Extremely Low Income } \\
\text { and Very Low Income) }\end{array}$ & $\begin{array}{l}\text { At or Below 80\% AMI } \\
\text { (Very Low Income, } \\
\text { Extremely Low Income, } \\
\text { and Very Low Income) }\end{array}$ \\
\hline Birmingham, AL & 37 & 81 & 106 \\
\hline Huntsville, AL & 48 & 96 & 115 \\
\hline Mobile, AL & 38 & 65 & 115 \\
\hline Montgomery, AL & 25 & 51 & 103 \\
\hline Tuscaloosa, AL & 32 & 60 & 95 \\
\hline Cape Coral, FL & 5 & 13 & 91 \\
\hline Fort Lauderdale, $\mathrm{FL}^{*}$ & 12 & 15 & 58 \\
\hline Gainesville, FL & 29 & 68 & 100 \\
\hline Hialeah, FL & 18 & 25 & 32 \\
\hline Jacksonville, FL & 32 & 45 & 101 \\
\hline Miami, FL & 26 & 30 & 47 \\
\hline Orlando, FL & 19 & 27 & 87 \\
\hline Port St Lucie, FL & 38 & 23 & 79 \\
\hline St Petersburg, FL & 23 & 31 & 85 \\
\hline Tallahassee, FL & 16 & 51 & 105 \\
\hline Tampa, FL & 27 & 33 & 89 \\
\hline Athens-Clarke County, GA & 18 & 30 & 86 \\
\hline Atlanta, GA & 33 & 59 & 98 \\
\hline Augusta-Richmond County, GA & 30 & 56 & 105 \\
\hline Columbus, GA & 24 & 50 & 84 \\
\hline Macon-Bibb County, GA & 33 & 62 & 100 \\
\hline Savannah, GA & 31 & 40 & 81 \\
\hline Baton Rouge, LA & 16 & 71 & 113 \\
\hline New Orleans, LA & 23 & 40 & 86 \\
\hline Jackson, MS & 15 & 38 & 104 \\
\hline Chattanooga, TN & 34 & 69 & 110 \\
\hline Knoxville, TN & 46 & 67 & 102 \\
\hline Memphis, TN & 21 & 58 & 104 \\
\hline Nashville-Davidson County, TN & 31 & 47 & 98 \\
\hline
\end{tabular}

Source: Authors' tabulations of U.S. Census Bureau's 2015 American Community Survey public use microdata sample (PUMS) data *Fort Lauderdale, Florida, area also includes Pompano Beach, Tamarac, and Oakland Park. 
Units affordable and available per 100 renter households for households at 30 percent $\mathrm{AMI}$ and below (extremely low income) range from only five in Cape Coral, Florida, to 48 in Huntsville, Alabama. Units at 50 percent AMI and below (extremely low and very low income) range from only 13 in Cape Coral to 96 in Huntsville. Huntsville is an outlier due mainly to a relatively high AMI and larger share of upper-income households in the area. Figure 13 shows affordable units available at 50 percent AMI or below by city, sorted by greatest relative need.

\section{Figure 13: Affordable and Available Units per 100 Extremely Low- and Very Low-Income ( $<50 \%$ AMI) Tenants by City}

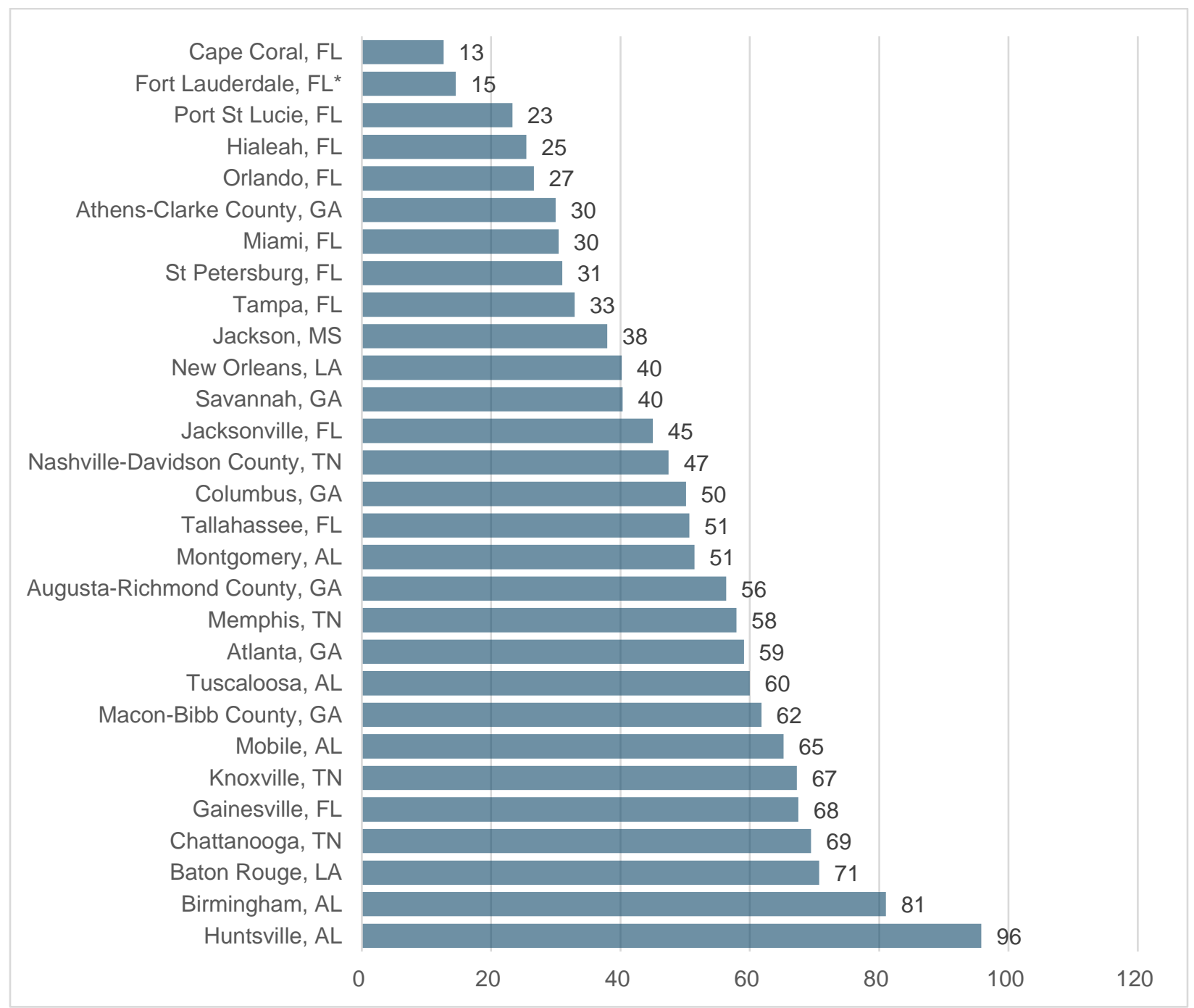

Source: Authors' tabulations of U.S. Census Bureau's 2015 American Community Survey public use microdata sample (PUMS) data *Fort Lauderdale, Florida, area also includes Pompano Beach, Tamarac, and Oakland Park.

There is great variation in the absolute surplus or deficit in units by city, which is influenced by the populations of these cities (see table 8). Many cities have a larger shortage at the level of 30 percent 
Atlanta Fed Community \& Economic Development Discussion Paper Series • No. 02-18

$\mathrm{AMI}$ and below (extremely low income) and others at the level of 50 percent $\mathrm{AMI}$ and below (extremely low income and very low income). Several cities had small surpluses at the level of 80 percent AMI and below, again indicating a relatively healthy supply of workforce housing. 
Table 8: Surplus or Deficit of Affordable and Available Units by AMI by City

\begin{tabular}{|c|c|c|c|}
\hline \multirow[b]{2}{*}{ City (Alphabetically by State) } & \multicolumn{3}{|c|}{ Surplus or Deficit of Affordable and Available Units } \\
\hline & $\begin{array}{l}\text { At or Below 30\% AMI } \\
\text { (Extremely Low Income) }\end{array}$ & $\begin{array}{l}\text { At or Below 50\% AMI } \\
\text { (Extremely Low Income } \\
\text { and Very Low Income) }\end{array}$ & $\begin{array}{l}\text { At or Below 80\% AMI } \\
\text { (Very Low Income, } \\
\text { Extremely Low Income, } \\
\text { and Very Low Income) }\end{array}$ \\
\hline Birmingham, AL & $-11,608$ & $-5,084$ & 2,131 \\
\hline Huntsville, AL & $-5,417$ & -749 & 3,753 \\
\hline Mobile, AL & $-6,505$ & $-6,136$ & 3,940 \\
\hline Montgomery, AL & $-7,734$ & $-8,886$ & 842 \\
\hline Tuscaloosa, AL & $-3,146$ & $-3,317$ & -685 \\
\hline Cape Coral, FL & $-2,310$ & $-5,314$ & -817 \\
\hline Fort Lauderdale, $\mathrm{FL}^{*}$ & $-11,408$ & $-22,015$ & $-17,242$ \\
\hline Gainesville, FL & $-8,086$ & $-5,289$ & -67 \\
\hline Hialeah, FL & $-6,300$ & $-11,250$ & $-14,931$ \\
\hline Jacksonville, FL & $-22,223$ & $-31,030$ & 501 \\
\hline Miami, FL & $-20,680$ & $-35,016$ & $-39,229$ \\
\hline Orlando, FL & $-13,351$ & $-29,570$ & $-8,113$ \\
\hline Port St Lucie, FL & $-1,214$ & $-2,403$ & $-1,362$ \\
\hline St Petersburg, FL & $-7,148$ & $-11,155$ & $-3,793$ \\
\hline Tallahassee, FL & $-11,777$ & $-10,726$ & 1,576 \\
\hline Tampa, FL & $-20,113$ & $-30,560$ & $-7,189$ \\
\hline Athens-Clarke County, GA & $-7,975$ & $-10,283$ & $-2,617$ \\
\hline Atlanta, GA & $-24,267$ & $-22,960$ & $-1,763$ \\
\hline Augusta-Richmond County, GA & $-7,180$ & $-6,564$ & 1,032 \\
\hline Columbus, GA & $-5,849$ & $-7,010$ & $-3,404$ \\
\hline Macon-Bibb County, GA & $-6,477$ & $-5,730$ & 27 \\
\hline Savannah, GA & $-5,619$ & $-8,489$ & $-4,206$ \\
\hline Baton Rouge, LA & $-8,031$ & $-4,597$ & 3,039 \\
\hline New Orleans, LA & $-20,972$ & $-24,604$ & $-7,507$ \\
\hline Jackson, MS & $-6,463$ & $-7,932$ & 674 \\
\hline Chattanooga, TN & $-6,848$ & $-5,939$ & 2,960 \\
\hline Knoxville, TN & $-6,655$ & $-6,509$ & 536 \\
\hline Memphis, TN & $-27,798$ & $-25,287$ & 3,017 \\
\hline Nashville-Davidson County, TN & $-17,238$ & $-24,576$ & $-1,571$ \\
\hline
\end{tabular}

Source: Authors' tabulations of U.S. Census Bureau's 2015 American Community Survey public use microdata sample (PUMS) data *Fort Lauderdale, Florida, area also includes Pompano Beach, Tamarac, and Oakland Park. 
The shortage of affordable and available units at 50 percent AMI and below in absolute numbers is shown in figure 14, sorted from greatest to least. The deficit ranged from 749 units in Huntsville to 35,016 units in Miami. These numbers are a reasonable approximation; however, we again note the PUMA boundaries for cities, like MSAs, are not an exact fit. Therefore, the calculations may contain neighborhoods outside of or exclude neighborhoods in the city limits. Given their populations, large cities such as Miami, New Orleans, Nashville, and Atlanta had among the largest deficits in the Southeast for extremely low- and very low-income levels. Cities in Florida also had high absolute deficits in affordable and available units at these income levels.

\section{Figure 14: Deficit of Affordable and Available Units for Extremely Low- and Very Low- Income ( $<50 \%$ AMI) Households by City}

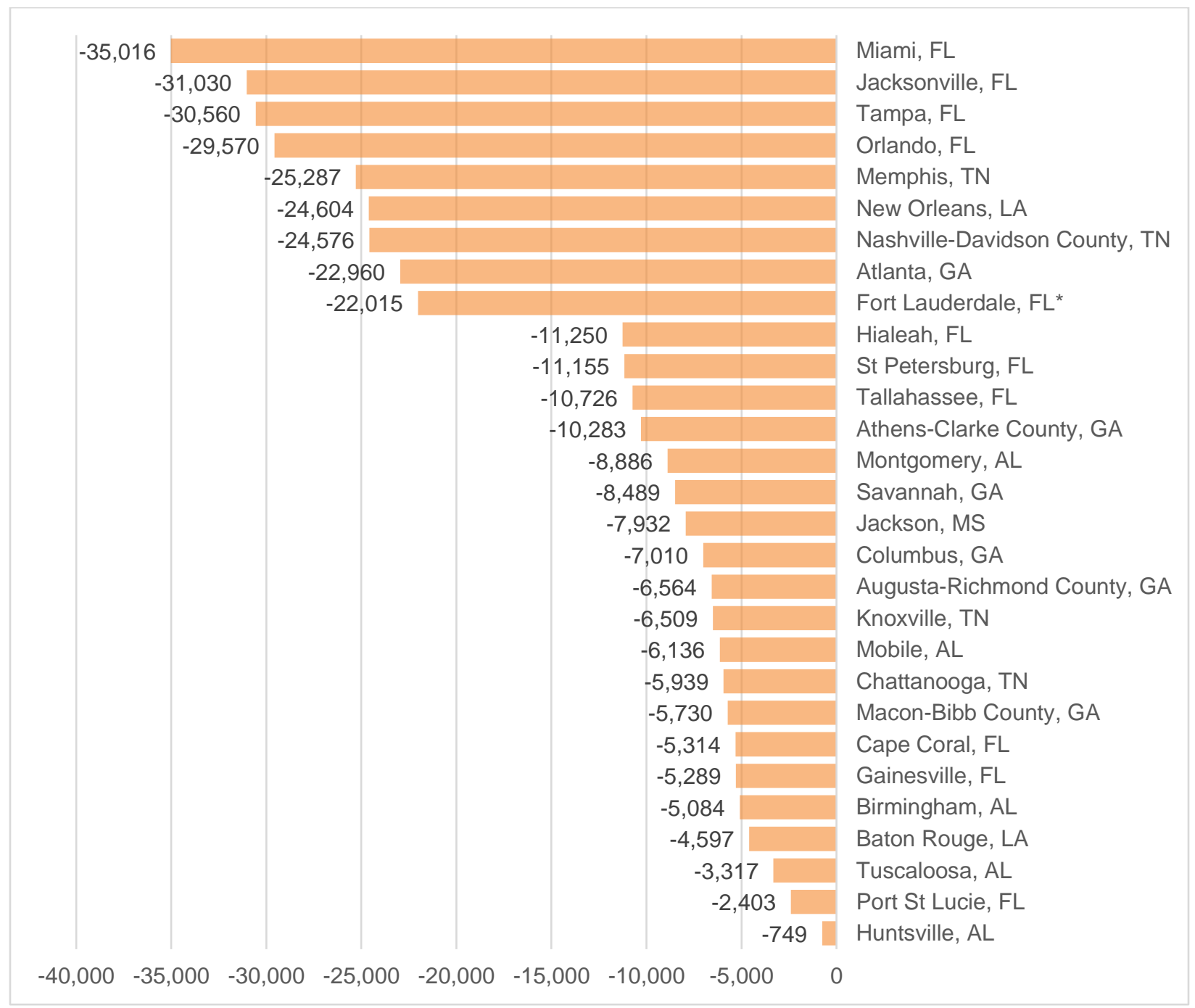

Source: Authors' tabulations of U.S. Census Bureau's 2015 American Community Survey public use microdata sample (PUMS) data *Fort Lauderdale, Florida, area also includes Pompano Beach, Tamarac, and Oakland Park. 


\section{Conclusion}

The above data demonstrate the abundance of renter households that are cost burdened as well as the extraordinary need for additional affordable rental units, particularly at rents affordable to extremely low- and very low-income households, in the Atlanta Fed's District. This is generally due to rents increasing at a greater pace than income (particularly among low-cost rentals), a higher demand for rental housing, and the loss of low-cost subsidized and naturally occurring affordable rental housing units. Although the magnitude of the problem is most acute in Florida and in large metropolitan areas, the need is great throughout the Southeast, from rural areas to dense urban centers. A surge in need has also been experienced in disaster-affected areas of the district, such as after the 2016 floods in and around Baton Rouge, Louisiana, and after Hurricanes Irma and Maria in 2017.

Potential solutions for this widespread problem include increased resources dedicated to affordable housing, realignment of local planning and policy to support affordable housing development, and greater tenant protections, among others. We outline high-level strategies for addressing the current affordable housing crisis.

Five of six states and eight local jurisdictions in the Atlanta Fed's District have established a housing trust fund (Center for Community Change, 2016), although funding levels appear to be inadequate given the shortage of units presented in this report. Florida, for example, has the most stable and robust source of dedicated funding among the six states through its document stamp tax on real estate transactions. However, a significant portion of the funds has been routinely diverted to other activities. Alabama also has a statewide housing trust fund, although no funds are allocated to the fund. Mississippi is the sole state in the district that does not currently have a state housing trust fund. At the least, legislation could be passed to establish a trust fund in every state. In addition to real estate transfer taxes, other potential sources of funding for trust funds include general revenue, short-term rental fees, impact fees, and employer taxes based on revenue and number of employees. Funds should be flexible, but activities such as acquisition and predevelopment and preservation could be prioritized due to the present high cost of land and the mounting losses of affordable units.

Land and other input costs have been increasing in recent years, limiting the ability to produce affordable housing. Further, exclusionary land use and zoning policies and building code regulations impart additional costs of development. For example, density restrictions, parking requirements, and building material standards that preclude lower-cost options may make affordable multifamily projects impossible or prohibitively expensive to build (Glover, Carpenter, \& Duckworth, 2017). When possible, such standards could be eliminated or waived, and they could provide the important public benefit of affordable housing. Donated or public land could be utilized for the development of affordable housing to defer the rapidly increasing cost of land (Spotts, Hale-Case, \& Abu-Khalaf, 2017).

Inclusionary housing policies can increase the stock of affordable housing depending on the market conditions and availability of incentives (Thaden \& Wang, 2017; Williams, 2016). Done well, they could potentially increase the production of affordable housing without limiting the production of market-rate housing. However, there is some risk that the additional regulation could lower aggregate housing supply. Several cities in the Southeast have considered inclusionary zoning, or a mandatory or 
voluntary set aside of affordable units in any multifamily project. Only a handful of local jurisdictions in the Southeast have successfully adopted such policies, and they have done so in a fairly limited manner. For example, at the time of this publication, Atlanta's inclusionary zoning ordinance is limited to certain neighborhoods, and Miami has a voluntary rather than mandatory policy. State legislatures ultimately blocked ordinances in Nashville and Louisiana. While inclusionary housing policies are but one tool in an affordable housing toolbox and are unlikely to achieve the level of production required to address the current gap in units, such policies could be effectively crafted with input from the developer community to increase access to affordable housing, particularly in higher-cost submarkets.

To stabilize housing for lower-income households, greater tenant-side protections could be considered. They might include a ban on rent hikes on units that do not meet livability standards (as adopted by Seattle), just cause eviction laws, and greater access to legal aid or a guaranteed attorney for tenants facing eviction. Tenant opportunity-to-purchase, which allow residents to buy their property directly or through a nonprofit intermediary, could also preserve affordable units and keep residents in place. Source of income discrimination, where landlords have the ability to deny renters who receive public assistance such as HUD's housing choice vouchers, could be prohibited.

Just as tenants should be supported, landlords who wish to maintain their affordable units could be incentivized to do so. This might include property tax breaks in exchange for a longer-term affordability commitment, affordable financing for purchase and renovation, or other forms of support. In many markets, privately owned small and medium multifamily structures with less than 50 units form a sizable proportion of unsubsidized or naturally occurring affordable rental housing (An, Bostic, Jakabovics, Orlando, \& Rodnyansky, 2017). Given this need, innovations are under way to preserve these units. An example is the ATL Challenge, an Atlanta affordable housing preservation grant competition won by nonprofit housing developer Tapestry Development Group (TDG). TDG received a grant to develop a low-cost loan moderate rehabilitation fund for owners or midsized multifamily properties serving residents at 80 percent $\mathrm{AMI}$ and below, tied to an affordability requirement of 10 years or the term of the loan (Schreiber, 2018).

The inability of families to find and secure affordable housing hinges on both a lack of low-cost supply as well as a lack of sufficient income. Therefore, housing experts could work closely with workforce development providers as well as employers to ensure that housing is located near services and employment opportunities or near public transportation to access those opportunities.

Homeownership models for low-income households could also be considered in order to provide additional options and allow for asset building among this population. Community land trusts, where land is held in trust and the structure is purchased by the occupant, are a way to maintain affordability while allowing a limited amount of equity to build and be transferred among generations (Davis, 2006).

In order to tackle the enormous challenge of increasing the supply of affordable housing and to achieve a better balance of housing options, state and local action is needed. While federal policies and federal subsidies are important, levels of federal funding are insufficient to meet demand, as only 21 percent of low-income renters with housing needs received assistance in 2015 (Kingsley, 2017). Thus, it 
is important first to define the problem and develop a cohesive vision for addressing it at the local level. Once the problem is understood, local and state stakeholders should build consensus on what works in the current environment and identify the resources or capacity to deploy these strategies at scale. Collectively, southeastern cities need more subsidies, incentives, greater coordination with other sectors (including public-private partnerships), and regional cooperation among neighboring jurisdictions.

Mixed-income communities that include affordable housing confer a variety of household and community benefits. Recent studies evaluating low-income housing tax credit (LIHTC) developments found improved public safety among high-crime neighborhoods (Woo \& Joh, 2015) and greater housing stability as well as access to employment and educational opportunities among residents in California (Reid, 2018). Given the large shares of cost-burdened renter households and the massive shortage of affordable and available units, much work lies ahead to improve access to similar high-quality affordable housing options in the Southeast. 


\section{Appendix A: Combined PUMA Regions, Counties or Parishes Included in Each Region, and Area Median Income Used for Calculations}

The tables below include combined PUMA regions constructed for this analysis. Names include the MSA, micropolitan statistical area $(\mu \mathrm{SA})$, and counties used to create the regions. The tables also show the counties or parishes included in each region and the AMI used for calculations. The tables are broken out by state. Statewide maps of these regions follow the tables.

\section{Alabama Combined PUMA Regions Used for Analysis}

\begin{tabular}{|c|c|c|c|}
\hline State & Combined PUMA Region Name & Counties in Region & $\begin{array}{l}2015 \text { ACS } \\
\text { AMI Used }\end{array}$ \\
\hline $\mathrm{AL}$ & Alexander City (micropolitan statistical area) & Tallapoosa & $\$ 51,475$ \\
\hline $\mathrm{AL}$ & Anniston-Oxford-Jacksonville (metropolitan statistical area) & Calhoun & $\$ 54,068$ \\
\hline AL & $\begin{array}{l}\text { Atmore (micropolitan statistical area) plus Choctaw, Clarke, Conecuh, Monroe, } \\
\text { Washington, and Wilcox Counties }\end{array}$ & $\begin{array}{l}\text { Choctaw, Clarke, Conecuh, } \\
\text { Escambia, Monroe, Washington, } \\
\text { Wilcox }\end{array}$ & $\$ 46,979$ \\
\hline$A L$ & Auburn-Opelika (metropolitan statistical area) & Lee & $\$ 63,080$ \\
\hline \multirow{2}{*}{$\mathrm{AL}$} & \multirow{2}{*}{$\begin{array}{l}\text { Birmingham-Hoover (metropolitan statistical area) plus Fayette, Lamar } \\
\text { Counties, and southern Marion County minus Chilton and Bibb Counties }\end{array}$} & $\begin{array}{l}\text { Part 1: Blount, Jefferson, St. Clair, } \\
\text { Shelby }\end{array}$ & $\$ 45,057$ \\
\hline & & $\begin{array}{l}\text { Part 2: Fayette, Lamar, Marion, } \\
\text { Walker }\end{array}$ & $\$ 68,086$ \\
\hline AL & $\begin{array}{l}\text { Columbus, GA-AL (metropolitan statistical area) plus Eufaula and Troy } \\
\text { (micropolitan statistical areas) and Bullock and Macon Counties }\end{array}$ & $\begin{array}{l}\text { Barbour, Bullock, Macon, Pike, } \\
\text { Russell }\end{array}$ & $\$ 45,057$ \\
\hline $\mathrm{AL}$ & Cullman (micropolitan statistical area) plus Winston County & Cullman, Winston & $\$ 42,053$ \\
\hline $\mathrm{AL}$ & Daphne-Fairhope-Foley (metropolitan statistical area) & Baldwin & $\$ 68,086$ \\
\hline $\mathrm{AL}$ & Decatur (metropolitan statistical area) & Lawrence, Morgan & $\$ 58,073$ \\
\hline $\mathrm{AL}$ & Dothan (metropolitan statistical area) plus Ozark (micropolitan statistical area) & Dale, Geneva, Henry, Houston & $\$ 53,568$ \\
\hline AL & $\begin{array}{l}\text { Enterprise (micropolitan statistical area) plus Butler, Covington, and Crenshaw } \\
\text { Counties }\end{array}$ & Butler, Coffee, Covington, Crenshaw & $\$ 50,464$ \\
\hline $\mathrm{AL}$ & $\begin{array}{l}\text { Florence-Muscle Shoals (metropolitan statistical area) plus Franklin County and } \\
\text { northern Marion County }\end{array}$ & $\begin{array}{l}\text { Colbert, Franklin, Lauderdale, } \\
\text { Marion }\end{array}$ & $\$ 53,067$ \\
\hline
\end{tabular}




\begin{tabular}{|l|l|l|l|}
\hline AL & $\begin{array}{l}\text { Fort Payne (micropolitan statistical area) plus Scottsboro (micropolitan } \\
\text { statistical area) }\end{array}$ & DeKalb, Jackson & \$43,355 \\
\hline AL & Gadsden (metropolitan statistical area) & Etowah & L50,864 \\
\hline AL & $\begin{array}{l}\text { Huntsville (metropolitan statistical area) plus Albertville (micropolitan } \\
\text { statistical area) }\end{array}$ & Limestone, Madison, Marshall & \$68,286 \\
\hline AL & Mobile (metropolitan statistical area) & $\begin{array}{l}\text { Autauga, Elmore, Lowndes, } \\
\text { Montgomery }\end{array}$ & $\$ 56,471$ \\
\hline AL & Montgomery (metropolitan statistical area) & $\begin{array}{l}\text { Chambers, Cherokee, Chilton, Clay, } \\
\text { Cleburne, Coosa, Randolph, } \\
\text { Talladega }\end{array}$ & \$48,561 \\
\hline AL & $\begin{array}{l}\text { Talladega-Sylacauga (micropolitan statistical area) plus Valley (micropolitan } \\
\text { statistical area) and Chilton, Clay, Cleburne, and Randolph Counties }\end{array}$ \\
\hline AL & $\begin{array}{l}\text { Tuscaloosa (metropolitan statistical area) plus Selma (micropolitan statistical } \\
\text { area) and Bibb, Greene, Marengo, Perry, and Sumter Counties }\end{array}$ & $\begin{array}{l}\text { Bibb, Dallas, Greene, Hale, Marengo, } \\
\text { Perry, Pickens, Sumter, Tuscaloosa }\end{array}$ & $\mathbf{\$ 5 4 , 2 6 9}$ \\
\hline
\end{tabular}

\section{Florida Combined PUMA Regions Used for Analysis}

\begin{tabular}{|l|l|l|l|}
\hline State & Combined PUMA Region Name & Counties in Region & $\begin{array}{l}\mathbf{2 0 1 5} \text { ACS } \\
\text { AMI Used }\end{array}$ \\
\hline FL & Cape Coral-Fort Myers (metropolitan statistical area) & Lee & $\$ 60,106$ \\
\hline FL & $\begin{array}{l}\text { Crestview-Fort Walton Beach-Destin (metropolitan statistical area) minus } \\
\text { Walton County }\end{array}$ & Okaloosa & \$61,978 \\
\hline FL & $\begin{array}{l}\text { Deltona-Daytona Beach-Ormond Beach (metropolitan statistical area) plus } \\
\text { Palm Coast (metropolitan statistical area) }\end{array}$ & Flagler, Volusia & \$52,066 \\
\hline FL & Fort Lauderdale & Broward & \$62,279 \\
\hline FL & Gainesville (metropolitan statistical area) minus Gilchrist County & Alachua & \$68,086 \\
\hline FL & Homosassa Springs (metropolitan statistical area) & Citrus & \$49,162 \\
\hline FL & $\begin{array}{l}\text { Jacksonville (metropolitan statistical area) plus Palatka (micropolitan statistical } \\
\text { area) }\end{array}$ & $\begin{array}{l}\text { Baker, Clay, Duval, Nassau, Putnam, } \\
\text { St. Johns }\end{array}$ & $\$ 64,501$ \\
\hline FL & $\begin{array}{l}\text { Lake City (micropolitan statistical area) plus Bradford, Dixie, Gilchrist, } \\
\text { Hamilton, Lafayette, Levy, Madison, Suwannee, Taylor, and Union Counties }\end{array}$ & $\begin{array}{l}\text { Bradford, Columbia, Dixie, Gilchrist, } \\
\text { Sumilton, Lafayette, Levy, Madison, }\end{array}$ & \$49,763 \\
\hline FL & Lakeland-Winter Haven (metropolitan statistical area) & Polk & Miami-Dade, Monroe \\
\hline FL & Miami-Dade plus Key West (micropolitan statistical area) & & \$51,064 \\
\hline
\end{tabular}




\begin{tabular}{|c|c|c|c|}
\hline & & & $\$ 67,986$ \\
\hline $\mathrm{FL}$ & Naples-Immokalee-Marco Island (metropolitan statistical area) & Collier & $\$ 70,088$ \\
\hline $\mathrm{FL}$ & North Port-Sarasota-Bradenton (metropolitan statistical area) & Manatee, Sarasota & $\$ 65,483$ \\
\hline $\mathrm{FL}$ & Ocala (metropolitan statistical area) & Marion & $\$ 50,464$ \\
\hline $\mathrm{FL}$ & $\begin{array}{l}\text { Orlando-Kissimmee-Sanford (metropolitan statistical area) plus The Villages } \\
\text { (metropolitan statistical area) }\end{array}$ & $\begin{array}{l}\text { Lake, Orange, Osceola, Seminole, } \\
\text { Sumter }\end{array}$ & $\$ 60,076$ \\
\hline $\mathrm{FL}$ & Palm Bay-Melbourne-Titusville (metropolitan statistical area) & Brevard & $\$ 62,078$ \\
\hline $\mathrm{FL}$ & $\begin{array}{l}\text { Panama City (metropolitan statistical area) plus Calhoun, Franklin, Gadsden, } \\
\text { Holmes, Jackson, Jefferson, Liberty, Wakulla, Walton, and Washington } \\
\text { Counties }\end{array}$ & $\begin{array}{l}\text { Bay, Calhoun, Franklin, Gadsden, } \\
\text { Gulf, Holmes, Jackson, Jefferson, } \\
\text { Liberty, Wakulla, Walton, } \\
\text { Washington }\end{array}$ & $\$ 51,765$ \\
\hline FL & Pensacola-Ferry Pass-Brent (metropolitan statistical area) & Escambia, Santa Rosa & $\$ 61,077$ \\
\hline $\mathrm{FL}$ & Port St. Lucie (metropolitan statistical area) & Martin, St. Lucie & $\$ 55,070$ \\
\hline $\mathrm{FL}$ & Punta Gorda (metropolitan statistical area) & Charlotte & $\$ 57,172$ \\
\hline $\mathrm{FL}$ & Sebastian-Vero Beach (metropolitan statistical area) & Indian River & $\$ 62,379$ \\
\hline $\mathrm{FL}$ & $\begin{array}{l}\text { Sebring (metropolitan statistical area) plus Arcadia, Clewiston, Okeechobee, } \\
\text { and Wauchula (micropolitan statistical areas) and Glades County }\end{array}$ & $\begin{array}{l}\text { DeSoto, Glades, Hardee, Hendry, } \\
\text { Highlands, Okeechobee }\end{array}$ & $\$ 42,654$ \\
\hline $\mathrm{FL}$ & $\begin{array}{l}\text { Tallahassee (metropolitan statistical area) minus Gadsden, Jefferson, and } \\
\text { Wakulla Counties }\end{array}$ & Leon & $\$ 70,990$ \\
\hline $\mathrm{FL}$ & Tampa-St. Petersburg-Clearwater (metropolitan statistical area) & $\begin{array}{l}\text { Hernando, Hillsborough, Pasco, } \\
\text { Pinellas }\end{array}$ & $\$ 60,276$ \\
\hline $\mathrm{FL}$ & West Palm Beach-Boca Raton & Palm Beach & $\$ 71,294$ \\
\hline
\end{tabular}

\section{Georgia Combined PUMA Regions Used for Analysis}

\begin{tabular}{|l|l|l|l|}
\hline State & Combined PUMA Region Name & $\begin{array}{l}\mathbf{2 0 1 5} \text { ACS } \\
\text { AMI Used }\end{array}$ \\
\hline GA & $\begin{array}{l}\text { Albany (metropolitan statistical area) plus Bainbridge, Moultrie, and } \\
\text { Thomasville (micropolitan statistical areas) and Calhoun, Early, Grady, Miller, } \\
\text { Mitchell, and Seminole Counties }\end{array}$ & $\begin{array}{l}\text { Baker, Calhoun, Colquitt, Decatur, } \\
\text { Dougherty, Early, Grady, Lee, Miller, } \\
\text { Mitchell, Seminole, Terrell, Thomas, } \\
\text { Worth }\end{array}$ & \$45,057 \\
\hline GA & $\begin{array}{l}\text { Athens-Clarke County (metropolitan statistical area) plus Elbert and Greene } \\
\text { Counties }\end{array}$ & $\begin{array}{l}\text { Clarke, Elbert, Greene, Madison, } \\
\text { Oconee, Oglethorpe }\end{array}$ & \$51,064 \\
\hline
\end{tabular}




\begin{tabular}{|c|c|c|c|}
\hline GA & $\begin{array}{l}\text { Atlanta-Sandy Springs-Roswell (metropolitan statistical area) minus Haralson } \\
\text { and Pickens Counties plus Jefferson, LaGrange, and Thomaston (micropolitan } \\
\text { statistical areas) }\end{array}$ & $\begin{array}{l}\text { Barrow, Bartow, Butts, Carroll, } \\
\text { Cherokee, Clayton, Cobb, Coweta, } \\
\text { DeKalb, Douglas, Fayette, Forsyth, } \\
\text { Fulton, Gwinnett, Heard, Henry, } \\
\text { Jackson, Jasper, Lamar, Meriwether, } \\
\text { Morgan, Newton, Paulding, Pike, } \\
\text { Rockdale, Spalding, Troup, Upson, } \\
\text { Walton }\end{array}$ & $\$ 70,088$ \\
\hline \multirow[b]{2}{*}{ GA } & \multirow[b]{2}{*}{$\begin{array}{l}\text { Augusta-Richmond County, GA-SC (metropolitan statistical area) plus Glascock, } \\
\text { Jefferson, Jenkins, Taliaferro, Warren, Washington, and Wilkes Counties }\end{array}$} & Part 1: Columbia, Richmond & $\$ 40,651$ \\
\hline & & $\begin{array}{l}\text { Part 2: Burke, Glascock, Hancock, } \\
\text { Jefferson, Jenkins, Lincoln, McDuffie, } \\
\text { Taliaferro, Warren, Washington, } \\
\text { Wilkes }\end{array}$ & $\$ 60,075$ \\
\hline GA & $\begin{array}{l}\text { Brunswick (metropolitan statistical area) minus Brantley County plus St. Marys } \\
\text { (micropolitan statistical area) }\end{array}$ & Camden, Glynn, McIntosh & $\$ 54,869$ \\
\hline GA & $\begin{array}{l}\text { Chattanooga, TN-GA (metropolitan statistical area) plus Summerville } \\
\text { (micropolitan statistical area) }\end{array}$ & Catoosa, Chattooga, Dade, Walker & $\$ 52,066$ \\
\hline \multirow[b]{2}{*}{ GA } & \multirow[b]{2}{*}{$\begin{array}{l}\text { Columbus, GA-AL (metropolitan statistical area) plus Americus, Eufaula, AL-GA, } \\
\text { and Cordele (micropolitan statistical areas) and Clay, Dooley, Macon, } \\
\text { Randolph, Stewart, Talbot, Taylor, and Webster Counties }\end{array}$} & Part 1: Chattahoochee, Muscogee & $\$ 51,064$ \\
\hline & & $\begin{array}{l}\text { Part 2: Clay, Crisp, Dooly, Harris, } \\
\text { Macon, Marion, Quitman, Randolph, } \\
\text { Schley, Stewart, Sumter, Talbot, } \\
\text { Taylor, Webster }\end{array}$ & $\$ 47,059$ \\
\hline GA & $\begin{array}{l}\text { Cornelia (micropolitan statistical area) plus Toccoa (micropolitan statistical } \\
\text { area) and Banks, Franklin, and Hart Counties }\end{array}$ & $\begin{array}{l}\text { Banks, Franklin, Habersham, Hart, } \\
\text { Stephens }\end{array}$ & $\$ 48,962$ \\
\hline GA & $\begin{array}{l}\text { Dalton (metropolitan statistical area) plus Calhoun (micropolitan statistical } \\
\text { area) and Fannin, Gilmer, and Pickens Counties }\end{array}$ & $\begin{array}{l}\text { Fannin, Gilmer, Gordon, Murray, } \\
\text { Pickens, Whitfield }\end{array}$ & $\$ 55,070$ \\
\hline GA & $\begin{array}{l}\text { Dublin, GA (micropolitan statistical area) plus Bleckley, Candler, Dodge, } \\
\text { Emanuel, Treutlen, and Wilcox Counties }\end{array}$ & $\begin{array}{l}\text { Whitfield, Bleckley, Candler, Dodge, } \\
\text { Emanuel, Johnson, Laurens, } \\
\text { Treutlen, Wilcox }\end{array}$ & $\$ 44,356$ \\
\hline GA & Gainesville (metropolitan statistical area) & Hall & $\$ 60,076$ \\
\hline GA & Hinesville (metropolitan statistical area) plus Bryan County & Bryan, Liberty, Long & $\$ 47,059$ \\
\hline
\end{tabular}




\begin{tabular}{|c|c|c|c|}
\hline GA & $\begin{array}{l}\text { Macon-Bibb County (metropolitan statistical area) plus Warner Robins } \\
\text { (metropolitan statistical area) and Baldwin, Putnam, and Wilkinson Counties }\end{array}$ & $\begin{array}{l}\text { Baldwin, Bibb, Crawford, Houston, } \\
\text { Jones, Monroe, Peach, Pulaski, } \\
\text { Putnam, Twiggs, Wilkinson }\end{array}$ & $\$ 55,070$ \\
\hline GA & Northeast Georgia nonmetropolitan area & $\begin{array}{l}\text { Dawson, Lumpkin, Rabun, Towns, } \\
\text { Union, White }\end{array}$ & $\$ 52,867$ \\
\hline GA & $\begin{array}{l}\text { Rome (metropolitan statistical area) plus Cedartown (micropolitan statistical } \\
\text { area) and Haralson County }\end{array}$ & Floyd, Haralson, Polk & $\$ 49,462$ \\
\hline GA & $\begin{array}{l}\text { Savannah (metropolitan statistical area) minus Bryan County plus Statesboro } \\
\text { (micropolitan statistical area) and Screven County }\end{array}$ & $\begin{array}{l}\text { Bulloch, Chatham, Effingham, } \\
\text { Screven }\end{array}$ & $\$ 58,073$ \\
\hline GA & $\begin{array}{l}\text { Valdosta (metropolitan statistical area) plus Fitzgerald, Douglas, Tifton, and } \\
\text { Waycross (micropolitan statistical areas) and Atkinson, Bacon, Berrien, } \\
\text { Brantley, Charlton, Clinch, Cook, Irwin, and Turner Counties }\end{array}$ & $\begin{array}{l}\text { Atkinson, Bacon, Ben Hill, Berrien, } \\
\text { Brantley, Brooks, Charlton, Clinch, } \\
\text { Coffee, Cook, Echols, Irwin, Lanier, } \\
\text { Lowndes, Pierce, Tift, Turner, Ware }\end{array}$ & $\$ 42,053$ \\
\hline GA & $\begin{array}{l}\text { Vidalia (micropolitan statistical area) plus Jesup (micropolitan statistical area) } \\
\text { and Appling, Evans, Jeff Davis, Tattnall, Telfair, and Wheeler Counties }\end{array}$ & $\begin{array}{l}\text { Appling, Evans, Jeff Davis, } \\
\text { Montgomery, Tattnall, Telfair, } \\
\text { Toombs, Wayne, Wheeler }\end{array}$ & $\$ 48,061$ \\
\hline
\end{tabular}

\section{Louisiana Combined PUMA Regions Used for Analysis}

\begin{tabular}{|l|l|l|l|}
\hline State & Combined PUMA Region Name & $\begin{array}{l}\text { 2015 ACS } \\
\text { AMI Used }\end{array}$ \\
\hline LA & $\begin{array}{l}\text { Plexandria (metropolitan statistical area) plus Fort Polk South (micropolitan } \\
\text { statistical area) and Natchez, MS-LA (micropolitan statistical area) and } \\
\text { Avoyelles, Catahoula, LaSalle and Winn Parishes }\end{array}$ & $\begin{array}{l}\text { Avoyelles, Catahoula, Concordia, } \\
\text { Grant, LaSalle, Rapides, Vernon, } \\
\text { Winn }\end{array}$ & \$51,265 \\
\hline \multirow{2}{*}{ LA } & Baton Rouge (metropolitan statistical area) & $\begin{array}{l}\text { Ascension, East Baton Rouge, East } \\
\text { Feliciana, Iberville, Livingston, Pointe } \\
\text { Coupee, St. Helena, West Baton } \\
\text { Rouge, West Feliciana }\end{array}$ & \$70,088 \\
\hline LA & $\begin{array}{l}\text { Hammond (metropolitan statistical area) plus Bogalusa (micropolitan statistical } \\
\text { area) }\end{array}$ & Tangipahoa, Washington & \$57,913 \\
\hline LA & Houma-Thibodaux (metropolitan statistical area) plus Assumption Parish & Assumption, Lafourche, Terrebonne & \$53,938 \\
\hline \multirow{2}{*}{ LA } & $\begin{array}{l}\text { Lafayette (metropolitan statistical area) plus Morgan City (micropolitan } \\
\text { statistical area) }\end{array}$ & Part 1: Acadia, Vermilion \\
\cline { 3 - 4 } & $\begin{array}{l}\text { Part 2: Iberia, Lafayette, St. Martin, } \\
\text { St. Mary }\end{array}$ & \$61,077 \\
\hline
\end{tabular}




\begin{tabular}{|l|l|l|l|}
\hline LA & $\begin{array}{l}\text { Lake Charles (metropolitan statistical area) plus DeRidder (micropolitan } \\
\text { statistical area) and Jennings (micropolitan statistical area) and Allen Parish }\end{array}$ & $\begin{array}{l}\text { Allen, Beauregard, Calcasieu, } \\
\text { Cameron, Jefferson Davis }\end{array}$ & \$55,871 \\
\hline \multirow{2}{*}{ LA } & $\begin{array}{l}\text { Monroe (metropolitan statistical area) plus Bastrop (micropolitan statistical } \\
\text { area) and Caldwell, East Carroll, Franklin, Jackson, Madison, Richland, Tensas, } \\
\text { and West Carroll Parishes }\end{array}$ & $\begin{array}{l}\text { Part 1: Ouachita } \\
\text { Part 2: Caldwell, East Carroll, } \\
\text { Franklin, Jackson, Madison, } \\
\text { Morehouse, Richland, Tensas, } \\
\text { Union, West Carroll }\end{array}$ & $\$ 47,059$ \\
\hline LA & New Orleans-Metairie (metropolitan statistical area) & $\begin{array}{l}\text { Jefferson, Orleans, Plaquemines, St. } \\
\text { Bernard, St. Charles, St. James, St. } \\
\text { John the Baptist, St. Tammany }\end{array}$ & \$62,479 \\
\hline LA & Opelousas (micropolitan statistical area) plus Evangeline Parish & Evangeline, St. Landry & \$39,049 \\
\hline LA & $\begin{array}{l}\text { Ruston (micropolitan statistical area) plus Natchitoches (micropolitan } \\
\text { statistical area) and DeSoto, Bienville, Claiborne, Red River, and Sabine } \\
\text { Parishes }\end{array}$ & $\begin{array}{l}\text { Bienville, Claiborne, De Soto, } \\
\text { Lincoln, Natchitoches, Red River, } \\
\text { Sabine, }\end{array}$ & \$48,111 \\
\hline LA & Shreveport-Bossier City (metropolitan statistical area) minus DeSoto Parish & Bossier, Caddo, Webster & \$56,071 \\
\hline
\end{tabular}

\section{Mississippi Combined PUMA Regions Used for Analysis}

\begin{tabular}{|l|l|l|l|}
\hline State & Combined PUMA Region Name & Counties in Region & $\begin{array}{l}\mathbf{2 0 1 5} \text { ACS } \\
\text { AMI Used }\end{array}$ \\
\hline MS & $\begin{array}{l}\text { Brookhaven (micropolitan statistical area) plus McComb (micropolitan } \\
\text { statistical area) and Vicksburg (micropolitan statistical area) }\end{array}$ & $\begin{array}{l}\text { Adams, Amite, Claiborne, Franklin, } \\
\text { Jefferson, Lawrence, Lincoln, Pike, } \\
\text { Walthall, Wilkinson }\end{array}$ & \$40,051 \\
\hline MS & $\begin{array}{l}\text { Cleveland (micropolitan statistical area) plus Indianola (micropolitan statistical } \\
\text { area) and Greenville (micropolitan statistical area) }\end{array}$ & $\begin{array}{l}\text { Bolivar, Humphreys, Issaquena, } \\
\text { Sharkey, Sunflower, Washington }\end{array}$ & \$32,040 \\
\hline MS & $\begin{array}{l}\text { Columbus (micropolitan statistical area) plus Starkville (micropolitan statistical } \\
\text { area) and West Point (micropolitan statistical area) }\end{array}$ & $\begin{array}{l}\text { Clay, Lowndes, Noxubee, Oktibbeha, } \\
\text { Webster, Winston }\end{array}$ & \$46,058 \\
\hline MS & $\begin{array}{l}\text { Greenwood (micropolitan statistical area) plus Grenada (micropolitan } \\
\text { statistical area) }\end{array}$ & $\begin{array}{l}\text { Attala, Carroll, Grenada, Holmes, } \\
\text { Leflore, Montgomery, Yalobusha }\end{array}$ & \$41,252 \\
\hline MS & $\begin{array}{l}\text { Gulfport-Biloxi-Pascagoula (metropolitan statistical area) plus Picayune } \\
\text { (micropolitan statistical area) and George and Stone Counties }\end{array}$ & $\begin{array}{l}\text { George, Hancock, Harrison, Jackson, } \\
\text { Pearl River, Stone }\end{array}$ & \$52,066 \\
\hline MS & Hattiesburg (metropolitan statistical area) plus Marion County & Forrest, Lamar, Marion, Perry & \$51,355 \\
\hline MS & Jackson (metropolitan statistical area) & $\begin{array}{l}\text { Copiah, Hinds, Madison, Rankin, } \\
\text { Simpson, Warren, Yazoo }\end{array}$ & \$55,570 \\
\hline
\end{tabular}




\begin{tabular}{|l|l|l|l|}
\hline MS & Laurel (micropolitan statistical area) & $\begin{array}{l}\text { Covington, Greene, Jefferson Davis, } \\
\text { Jones, Wayne }\end{array}$ & \$41,252 \\
\hline MS & Memphis, TN-MS-AR (metropolitan statistical area) & $\begin{array}{l}\text { Alcorn, Benton, Coahoma, DeSoto, } \\
\text { Marshall, Panola, Prentiss, Quitman, } \\
\text { Tallahatchie, Tate, Tippah, } \\
\text { Tishomingo, Tunica }\end{array}$ \\
\hline MS & $\begin{array}{l}\text { Meridian (micropolitan statistical area) plus Laurel (micropolitan statistical } \\
\text { area) }\end{array}$ & $\begin{array}{l}\text { Clarke, Jasper, Kemper, Lauderdale, } \\
\text { Leake, Neshoba, Newton, Scott, } \\
\text { Smith }\end{array}$ \\
\hline MS & $\begin{array}{l}\text { Tupelo (micropolitan statistical area) plus Oxford (micropolitan statistical area) } \\
\text { \$4alhoun, Chickasaw, Itawamba, } \\
\text { Lafayette, Lee, Monroe, Pontotoc, } \\
\text { Union }\end{array}$ & \$50,063 \\
\hline
\end{tabular}

\section{Tennessee Combined PUMA Regions Used for Analysis}

\begin{tabular}{|c|c|c|c|}
\hline State & Combined PUMA Region Name & Counties in Region & $\begin{array}{l}2015 \text { ACS } \\
\text { AMI Used }\end{array}$ \\
\hline $\mathrm{TN}$ & $\begin{array}{l}\text { Brownsville (micropolitan statistical area) plus Decatur, Hardeman, Hardin, } \\
\text { Henderson, and McNairy Counties }\end{array}$ & $\begin{array}{l}\text { Decatur, Hardeman, Hardin, } \\
\text { Haywood, Henderson, McNairy }\end{array}$ & $\$ 45,057$ \\
\hline TN & $\begin{array}{l}\text { Chattanooga, TN-GA (metropolitan statistical area) plus Bledsoe, Grundy, } \\
\text { Meigs, and Rhea Counties }\end{array}$ & $\begin{array}{l}\text { Bledsoe, Grundy, Hamilton, Marion, } \\
\text { Meigs, Rhea, Sequatchie }\end{array}$ & $\$ 59,926$ \\
\hline TN & Clarksville, TN-KY (metropolitan statistical area) plus Stewart County & Montgomery, Stewart & $\$ 56,772$ \\
\hline TN & Cleveland (metropolitan statistical area) plus McMinn County & Bradley, McMinn, Polk & $\$ 53,287$ \\
\hline TN & Cookeville (micropolitan statistical area) plus Clay and Picket Counties & $\begin{array}{l}\text { Clay, Jackson, Overton, Pickett, } \\
\text { Putnam }\end{array}$ & $\$ 44,957$ \\
\hline $\mathrm{TN}$ & $\begin{array}{l}\text { Crossville (micropolitan statistical area) plus Fentress, Van Buren, and White } \\
\text { Counties }\end{array}$ & $\begin{array}{l}\text { Cumberland, Fentress, Van Buren, } \\
\text { White }\end{array}$ & $\$ 45,057$ \\
\hline \multirow[t]{2}{*}{$\mathrm{TN}$} & \multirow[t]{2}{*}{ Jackson (metropolitan statistical area) plus Crockett, Dyer, and Lake Counties } & $\begin{array}{l}\text { Part 1: Crockett, Dyer, Gibson, Lake, } \\
\text { Obion }\end{array}$ & $\$ 49,062$ \\
\hline & & Part 2: Chester, Madison & $\$ 56,571$ \\
\hline \multirow[t]{2}{*}{$\mathrm{TN}$} & \multirow[t]{2}{*}{ Johnson City (metropolitan statistical area) plus Johnson and Greene Counties } & $\begin{array}{l}\text { Part 1: Carter, Greene, Johnson, } \\
\text { Unicoi }\end{array}$ & $\$ 43,455$ \\
\hline & & Part 2: Washington & $\$ 54,068$ \\
\hline
\end{tabular}




\begin{tabular}{|c|c|c|c|}
\hline TN & Kingsport-Bristol-Bristol, TN-VA (metropolitan statistical area) & Hawkins, Sullivan & $\$ 51,265$ \\
\hline \multirow{2}{*}{ TN } & \multirow{2}{*}{$\begin{array}{l}\text { Knoxville (metropolitan statistical area) plus Claiborne, Hancock, Monroe, and } \\
\text { Scott Counties, minus Grainger County }\end{array}$} & $\begin{array}{l}\text { Part 1: Anderson, Blount, Knox, } \\
\text { Loudon, Monroe, Roane, Union }\end{array}$ & $\$ 63,080$ \\
\hline & & $\begin{array}{l}\text { Part 2: Campbell, Claiborne, } \\
\text { Hancock, Morgan, Scott }\end{array}$ & $\$ 42,854$ \\
\hline TN & $\begin{array}{l}\text { Lawrenceburg (micropolitan statistical area) plus Giles, Lewis, Perry, and } \\
\text { Wayne Counties }\end{array}$ & $\begin{array}{l}\text { Giles, Lawrence, Lewis, Perry, } \\
\text { Wayne }\end{array}$ & $\$ 45,057$ \\
\hline TN & $\begin{array}{l}\text { Martin (micropolitan statistical area) plus Paris (micropolitan statistical area) } \\
\text { and Benton, Carroll, Houston, and Humphreys Counties }\end{array}$ & $\begin{array}{l}\text { Benton, Carroll, Henry, Houston, } \\
\text { Humphreys, Weakley }\end{array}$ & $\$ 51,665$ \\
\hline TN & Memphis, TN-MS-AR (metropolitan statistical area) plus Lauderdale County & Fayette, Lauderdale, Shelby, Tipton & $\$ 60,076$ \\
\hline TN & $\begin{array}{l}\text { Morristown (metropolitan statistical area) plus Cocke, Grainger, and Sevier } \\
\text { Counties }\end{array}$ & $\begin{array}{l}\text { Cocke, Grainger, Hamblen, Jefferson, } \\
\text { Sevier }\end{array}$ & $\$ 51,064$ \\
\hline TN & $\begin{array}{l}\text { Nashville-Davidson--Murfreesboro--Franklin, TN (metropolitan statistical area) } \\
\text { plus Bedford, DeKalb, Marshall, and Warren Counties }\end{array}$ & $\begin{array}{l}\text { Bedford, Cannon, Cheatham, } \\
\text { Davidson, DeKalb, Dickson, Hickman, } \\
\text { Macon, Marshall, Maury, Robertson, } \\
\text { Rutherford, Smith, Sumner, } \\
\text { Trousdale, Warren, Williamson, } \\
\text { Wilson }\end{array}$ & $\$ 67,886$ \\
\hline TN & $\begin{array}{l}\text { Tullahoma-Manchester, TN (micropolitan statistical area) plus Lawrence } \\
\text { County }\end{array}$ & Coffee, Franklin, Lincoln, Moore & $\$ 52,066$ \\
\hline
\end{tabular}




\section{Map of Alabama Combined PUMA Regions Used for Analysis}

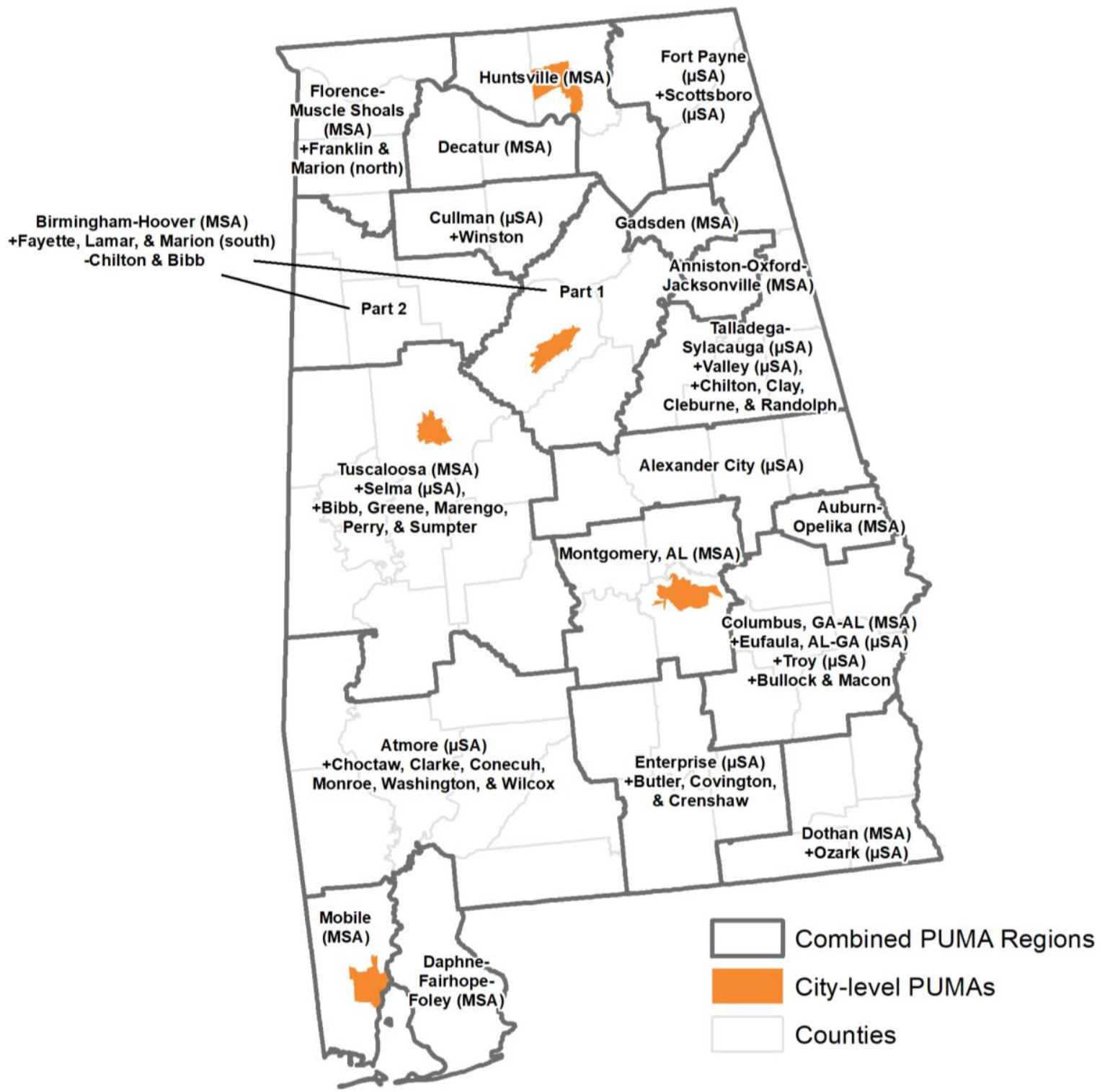




\section{Map of Georgia Combined PUMA Regions Used for Analysis}

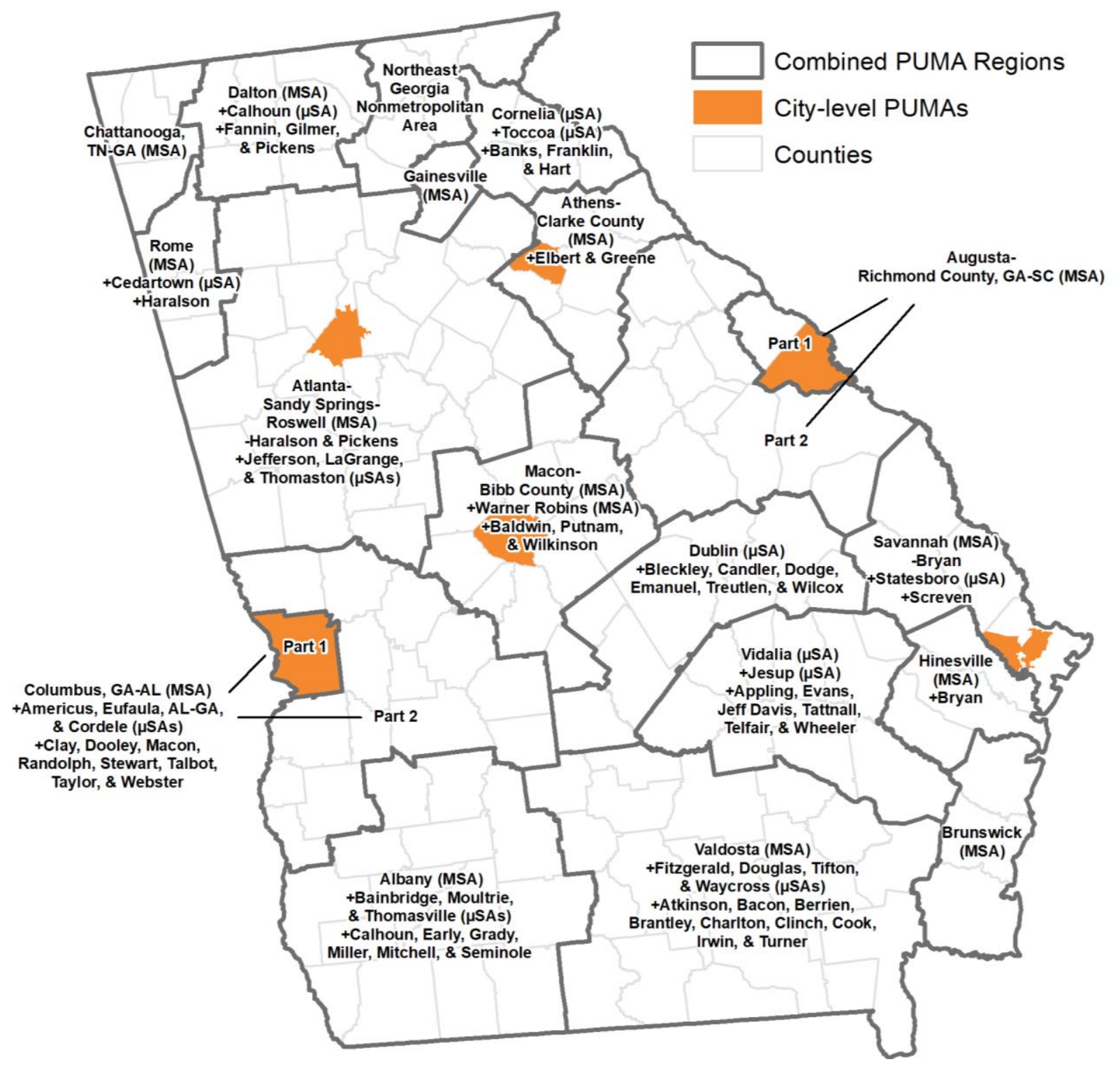


Map of Florida Combined PUMA Regions Used for Analysis

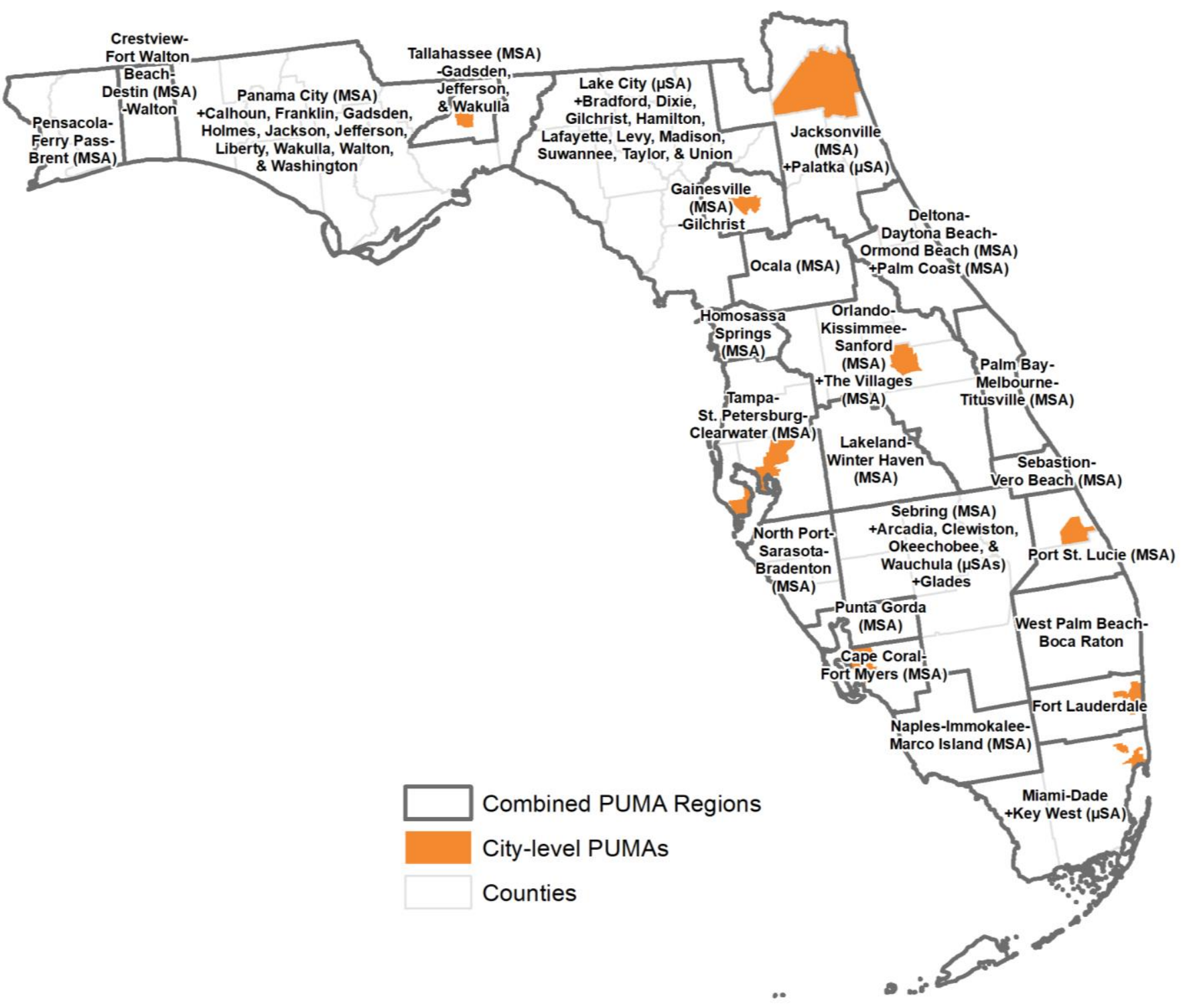




\section{Map of Louisiana Combined PUMA Regions Used for Analysis}

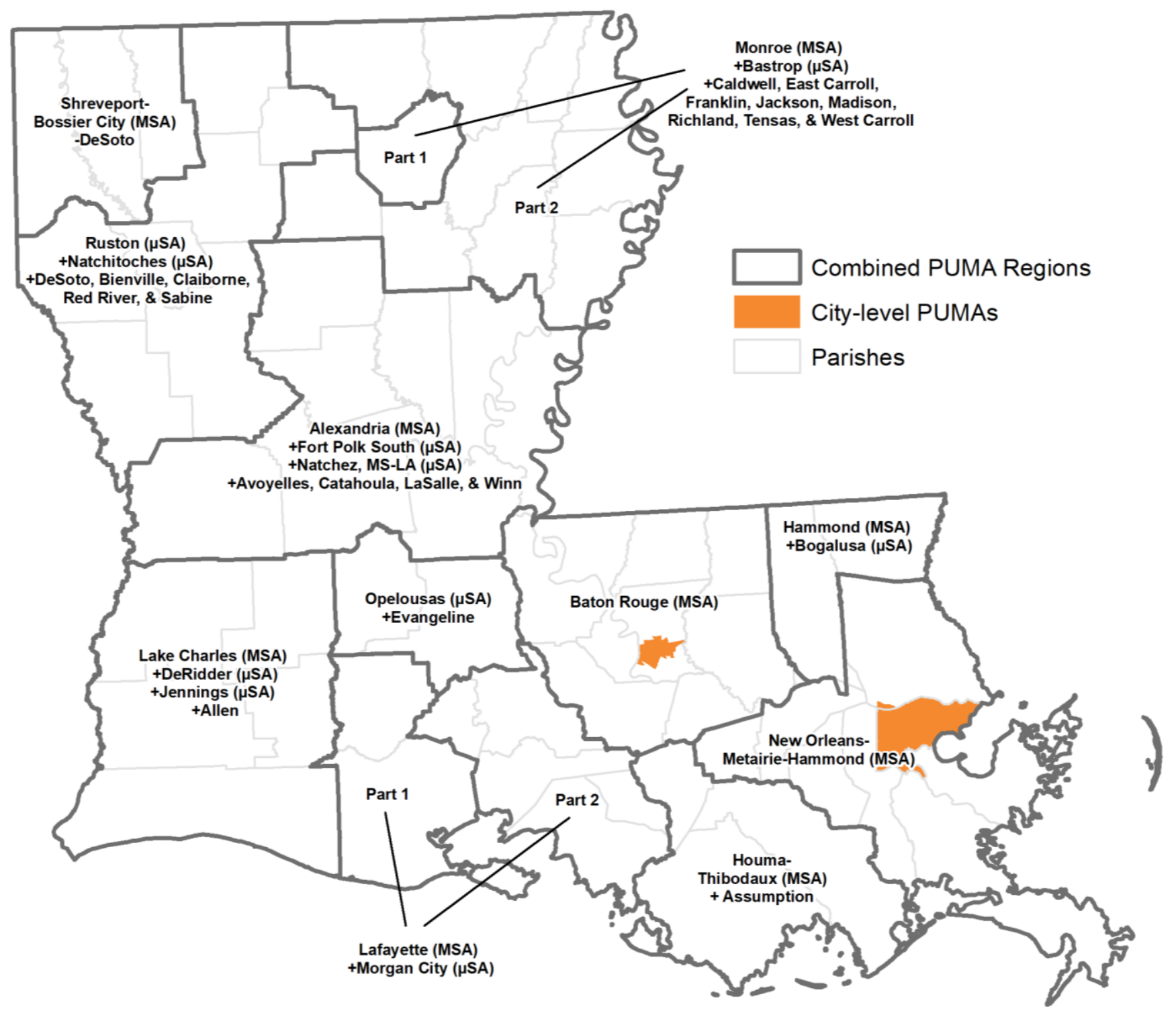




\section{Map of Mississippi Combined PUMA Regions Used for Analysis}

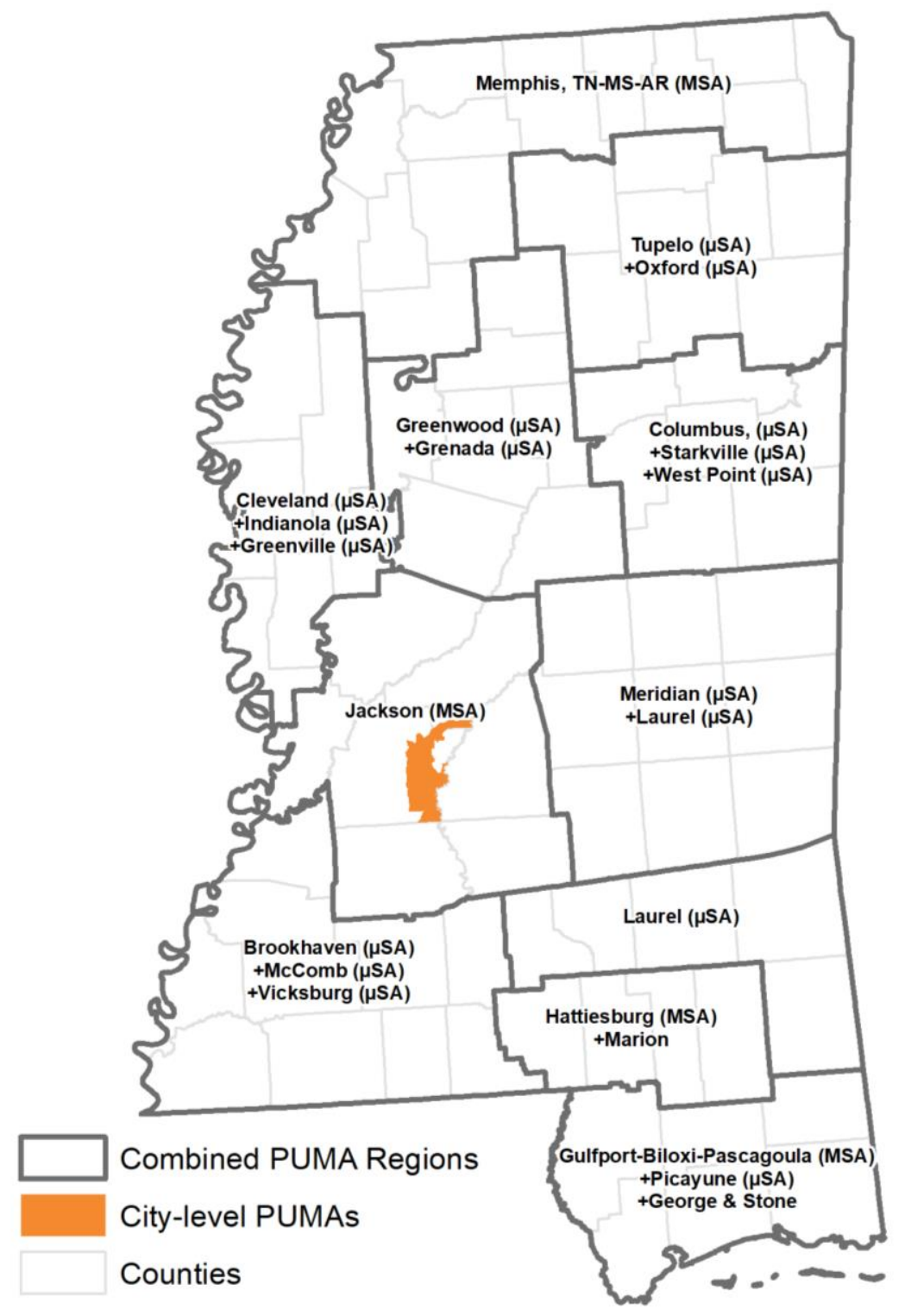




\section{Map of Tennessee Combined PUMA Regions Used for Analysis}

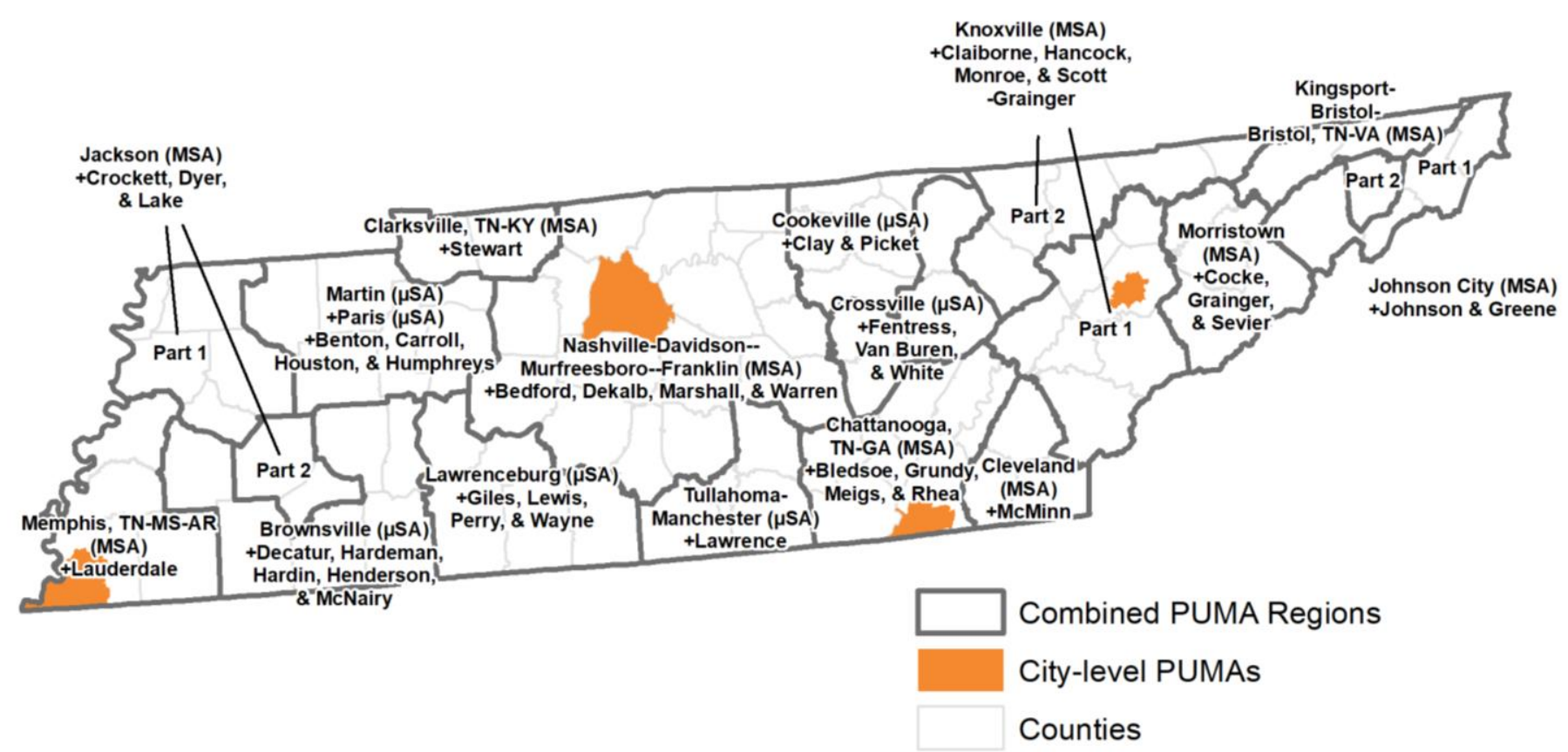


Appendix B: Full Data for All 99 Combined PUMA Regions: MSAs, Micropolitan Statistical Areas ( $\mu \mathrm{SAs}$ ), and Nonmetro Geographies

Number (and Percent) of Alabama Renter Households That Are Cost Burdened (Rent Is $>\mathbf{3 0}$ \% Household Income) by Income Category and by Combined PUMA Region

\begin{tabular}{|c|c|c|c|c|c|c|}
\hline & $\begin{array}{l}\text { Extremely } \\
\text { Low } \\
\text { Income } \\
\text { (30\% AMI } \\
\text { or Less) }\end{array}$ & $\begin{array}{l}\text { Very Low } \\
\text { Income } \\
\text { (30.01 to } \\
50 \% \text { AMI) }\end{array}$ & $\begin{array}{l}\text { Low } \\
\text { Income } \\
\text { (50.01 to } \\
80 \% \mathrm{AMI})\end{array}$ & $\begin{array}{l}\text { Moderate } \\
\text { Income } \\
\text { (80.01 to } \\
120 \% \text { AMI) }\end{array}$ & $\begin{array}{l}\text { Upper } \\
\text { Income } \\
\text { (More than } \\
120 \% \text { AMI) }\end{array}$ & $\begin{array}{l}\text { All Renter } \\
\text { House- } \\
\text { holds }\end{array}$ \\
\hline Alexander City $(\mu \mathrm{SA})$ & $\begin{array}{l}2,697 \\
(68 \%)\end{array}$ & $\begin{array}{l}2,215 \\
(72 \%)\end{array}$ & $\begin{array}{c}471 \\
(22 \%)\end{array}$ & $\begin{array}{l}158 \\
(9 \%)\end{array}$ & $\begin{array}{c}268 \\
(10 \%)\end{array}$ & $\begin{array}{l}5,809 \\
(43 \%)\end{array}$ \\
\hline $\begin{array}{l}\text { Anniston-Oxford- } \\
\text { Jacksonville (MSA) }\end{array}$ & $\begin{array}{l}3,198 \\
(80 \%)\end{array}$ & $\begin{array}{l}1,840 \\
(80 \%)\end{array}$ & $\begin{array}{c}983 \\
(35 \%)\end{array}$ & $\begin{array}{c}339 \\
(15 \%)\end{array}$ & $\begin{array}{c}0 \\
(0 \%)\end{array}$ & $\begin{array}{l}6,360 \\
(47 \%)\end{array}$ \\
\hline $\begin{array}{l}\text { Atmore }(\mu \mathrm{SA}) \text { plus Choctaw, } \\
\text { Clarke, Conecuh, Monroe, } \\
\text { Washington, and Wilcox } \\
\text { Counties }\end{array}$ & $\begin{array}{l}1,488 \\
(24 \%)\end{array}$ & $\begin{array}{c}989 \\
(35 \%)\end{array}$ & $\begin{array}{l}199 \\
(9 \%)\end{array}$ & $\begin{array}{c}70 \\
(3 \%)\end{array}$ & $\begin{array}{c}0 \\
(0 \%)\end{array}$ & $\begin{array}{l}2,746 \\
(17 \%)\end{array}$ \\
\hline Auburn-Opelika (MSA) & $\begin{array}{l}7,627 \\
(83 \%)\end{array}$ & $\begin{array}{l}2,633 \\
(66 \%)\end{array}$ & $\begin{array}{l}1,906 \\
(49 \%)\end{array}$ & $\begin{array}{l}159 \\
(4 \%)\end{array}$ & $\begin{array}{c}0 \\
(0 \%)\end{array}$ & $\begin{array}{c}12,325 \\
(51 \%)\end{array}$ \\
\hline $\begin{array}{l}\text { Birmingham-Hoover (MSA) } \\
\text { plus Fayette, Lamar } \\
\text { Counties and southern } \\
\text { Marion County minus } \\
\text { Chilton and Bibb Counties }\end{array}$ & $\begin{array}{c}30,042 \\
(74 \%)\end{array}$ & $\begin{array}{c}18,766 \\
(73 \%)\end{array}$ & $\begin{array}{l}8,476 \\
(34 \%)\end{array}$ & $\begin{array}{c}1,253 \\
(6 \%)\end{array}$ & $\begin{array}{c}364 \\
(2 \%)\end{array}$ & $\begin{array}{c}58,901 \\
(43 \%)\end{array}$ \\
\hline $\begin{array}{l}\text { Columbus, GA-AL (MSA) plus } \\
\text { Eufaula ( } \mu S A) \text { and Troy ( } \mu S A) \\
\text { and Bullock and Macon } \\
\text { Counties }\end{array}$ & $\begin{array}{l}4,666 \\
(69 \%)\end{array}$ & $\begin{array}{l}2,616 \\
(74 \%)\end{array}$ & $\begin{array}{l}2,500 \\
(53 \%)\end{array}$ & $\begin{array}{c}169 \\
(5 \%)\end{array}$ & $\begin{array}{l}174 \\
(4 \%)\end{array}$ & $\begin{array}{c}10,125 \\
(45 \%)\end{array}$ \\
\hline $\begin{array}{l}\text { Cullman ( } \mu \mathrm{SA}) \text { plus Winston } \\
\text { County }\end{array}$ & $\begin{array}{c}681 \\
(39 \%)\end{array}$ & $\begin{array}{c}259 \\
(30 \%)\end{array}$ & $\begin{array}{l}1,067 \\
(54 \%)\end{array}$ & $\begin{array}{c}563 \\
(24 \%)\end{array}$ & $\begin{array}{c}0 \\
0 \\
(0 \%)\end{array}$ & $\begin{array}{l}2,570 \\
(28 \%)\end{array}$ \\
\hline $\begin{array}{l}\text { Daphne-Fairhope-Foley } \\
\text { (MSA) }\end{array}$ & $\begin{array}{l}1,940 \\
(67 \%)\end{array}$ & $\begin{array}{l}2,957 \\
(57 \%)\end{array}$ & $\begin{array}{l}1,365 \\
(34 \%)\end{array}$ & $\begin{array}{c}588 \\
(18 \%)\end{array}$ & $\begin{array}{c}0 \\
(0 \%)\end{array}$ & $\begin{array}{l}6,850 \\
(35 \%)\end{array}$ \\
\hline Decatur (MSA) & $\begin{array}{l}2,238 \\
(47 \%)\end{array}$ & $\begin{array}{l}1,538 \\
(67 \%)\end{array}$ & $\begin{array}{l}250 \\
(5 \%)\end{array}$ & $\begin{array}{c}0 \\
(0 \%)\end{array}$ & $\begin{array}{l}113 \\
(5 \%)\end{array}$ & $\begin{array}{l}4,139 \\
(25 \%)\end{array}$ \\
\hline $\begin{array}{l}\text { Dothan (MSA) plus Ozark } \\
(\mu \mathrm{SA})\end{array}$ & $\begin{array}{l}4,414 \\
(74 \%)\end{array}$ & $\begin{array}{l}3,038 \\
(80 \%)\end{array}$ & $\begin{array}{l}1,628 \\
(32 \%)\end{array}$ & $\begin{array}{c}435 \\
(12 \%)\end{array}$ & $\begin{array}{c}13 \\
(0 \%)\end{array}$ & $\begin{array}{l}9,528 \\
(40 \%)\end{array}$ \\
\hline $\begin{array}{l}\text { Enterprise ( } \mu \mathrm{SA}) \text { plus Butler, } \\
\text { Covington, and Crenshaw } \\
\text { Counties }\end{array}$ & $\begin{array}{l}2,262 \\
(65 \%)\end{array}$ & $\begin{array}{l}1,137 \\
(54 \%)\end{array}$ & $\begin{array}{l}1,741 \\
(45 \%)\end{array}$ & $\begin{array}{l}178 \\
(8 \%)\end{array}$ & $\begin{array}{l}126 \\
(5 \%)\end{array}$ & $\begin{array}{l}5,444 \\
(38 \%)\end{array}$ \\
\hline $\begin{array}{l}\text { Florence-Muscle Shoals } \\
\text { (MSA) plus Franklin County }\end{array}$ & $\begin{array}{l}4,865 \\
(75 \%)\end{array}$ & $\begin{array}{l}2,389 \\
(52 \%)\end{array}$ & $\begin{array}{l}1,735 \\
(39 \%)\end{array}$ & $\begin{array}{l}229 \\
(7 \%)\end{array}$ & $\begin{array}{c}15 \\
(0 \%)\end{array}$ & $\begin{array}{l}9,233 \\
(40 \%)\end{array}$ \\
\hline
\end{tabular}




\begin{tabular}{|c|c|c|c|c|c|c|}
\hline $\begin{array}{l}\text { and northern Marion } \\
\text { County }\end{array}$ & & & & & & \\
\hline $\begin{array}{l}\text { Fort Payne }(\mu \mathrm{SA}) \text { plus } \\
\text { Scottsboro }(\mu \mathrm{SA})\end{array}$ & $\begin{array}{c}941 \\
(34 \%)\end{array}$ & $\begin{array}{l}1,685 \\
(62 \%) \\
\end{array}$ & $\begin{array}{l}1,112 \\
(44 \%) \\
\end{array}$ & $\begin{array}{c}524 \\
(18 \%) \\
\end{array}$ & $\begin{array}{c}91 \\
(2 \%) \\
\end{array}$ & $\begin{array}{l}4,353 \\
(28 \%) \\
\end{array}$ \\
\hline Gadsden (MSA) & $\begin{array}{l}1,143 \\
(58 \%)\end{array}$ & $\begin{array}{l}1,302 \\
(76 \%)\end{array}$ & $\begin{array}{c}827 \\
(52 \%)\end{array}$ & $\begin{array}{c}75 \\
(4 \%)\end{array}$ & $\begin{array}{c}0 \\
(0 \%)\end{array}$ & $\begin{array}{l}3,347 \\
(35 \%)\end{array}$ \\
\hline $\begin{array}{l}\text { Huntsville (MSA) plus } \\
\text { Albertville ( } \mu S A)\end{array}$ & $\begin{array}{c}12,504 \\
(76 \%)\end{array}$ & $\begin{array}{l}9,782 \\
(67 \%) \\
\end{array}$ & $\begin{array}{l}3,128 \\
(28 \%) \\
\end{array}$ & $\begin{array}{l}463 \\
(4 \%)\end{array}$ & $\begin{array}{c}0 \\
(0 \%)\end{array}$ & $\begin{array}{r}25,877 \\
(41 \%) \\
\end{array}$ \\
\hline Mobile (MSA) & $\begin{array}{l}9,905 \\
(79 \%)\end{array}$ & $\begin{array}{l}9,419 \\
(81 \%) \\
\end{array}$ & $\begin{array}{l}4,529 \\
(40 \%) \\
\end{array}$ & $\begin{array}{l}1,347 \\
(16 \%)\end{array}$ & $\begin{array}{c}64 \\
(1 \%) \\
\end{array}$ & $\begin{array}{c}25,264 \\
(47 \%) \\
\end{array}$ \\
\hline Montgomery (MSA) & $\begin{array}{r}10,687 \\
(68 \%) \\
\end{array}$ & $\begin{array}{l}8,182 \\
(81 \%) \\
\end{array}$ & $\begin{array}{l}4,127 \\
(47 \%) \\
\end{array}$ & $\begin{array}{l}1,273 \\
(14 \%) \\
\end{array}$ & $\begin{array}{c}15 \\
(0 \%) \\
\end{array}$ & $\begin{array}{r}24,284 \\
(46 \%) \\
\end{array}$ \\
\hline $\begin{array}{l}\text { Talladega-Sylacauga ( } \mu \mathrm{SA}) \\
\text { plus Valley ( } \mu \mathrm{SA} \text { ) and } \\
\text { Chilton, Clay, Cleburne, and } \\
\text { Randolph Counties }\end{array}$ & $\begin{array}{l}3,453 \\
(79 \%)\end{array}$ & $\begin{array}{l}1,799 \\
(46 \%)\end{array}$ & $\begin{array}{l}1,100 \\
(36 \%)\end{array}$ & $\begin{array}{c}155 \\
(5 \%)\end{array}$ & $\begin{array}{c}0 \\
(0 \%)\end{array}$ & $\begin{array}{l}6,507 \\
(36 \%)\end{array}$ \\
\hline $\begin{array}{l}\text { Tuscaloosa (MSA) plus } \\
\text { Selma ( } \mu \text { SA) and Bibb, } \\
\text { Greene, Marengo, Perry, } \\
\text { and Sumter Counties }\end{array}$ & $\begin{array}{l}9,401 \\
(70 \%)\end{array}$ & $\begin{array}{l}5,852 \\
(63 \%)\end{array}$ & $\begin{array}{l}3,505 \\
(42 \%)\end{array}$ & $\begin{array}{c}884 \\
(15 \%)\end{array}$ & $\begin{array}{c}96 \\
(1 \%)\end{array}$ & $\begin{array}{c}19,738 \\
(45 \%)\end{array}$ \\
\hline
\end{tabular}

\section{Number (and Percent) of Florida Renter Households That Are Cost Burdened (Rent Is} >30\% Household Income) by Income Category and by Combined PUMA Region

\begin{tabular}{|c|c|c|c|c|c|c|}
\hline & $\begin{array}{l}\text { Extremely } \\
\text { Low } \\
\text { Income } \\
\text { (30\% AMI } \\
\text { or Less) }\end{array}$ & $\begin{array}{l}\text { Very Low } \\
\text { Income } \\
\text { (30.01 to } \\
50 \% \text { AMI) }\end{array}$ & $\begin{array}{l}\text { Low } \\
\text { Income } \\
\text { (50.01 to } \\
80 \% \text { AMI) }\end{array}$ & $\begin{array}{l}\text { Moderate } \\
\text { Income } \\
\text { (80.01 to } \\
120 \% \text { AMI) }\end{array}$ & $\begin{array}{l}\text { Upper } \\
\text { Income } \\
\text { (More than } \\
120 \% \text { AMI) }\end{array}$ & $\begin{array}{c}\text { All Renter } \\
\text { House- } \\
\text { holds }\end{array}$ \\
\hline $\begin{array}{l}\text { Cape Coral-Fort Myers } \\
\text { (MSA) }\end{array}$ & $\begin{array}{c}10,526 \\
(71 \%)\end{array}$ & $\begin{array}{c}12,279 \\
(88 \%)\end{array}$ & $\begin{array}{c}11,052 \\
(66 \%)\end{array}$ & $\begin{array}{l}3,961 \\
(25 \%)\end{array}$ & $\begin{array}{c}1,472 \\
(8 \%)\end{array}$ & $\begin{array}{l}39,290 \\
(49 \%)\end{array}$ \\
\hline $\begin{array}{l}\text { Crestview-Fort Walton } \\
\text { Beach-Destin (MSA) minus } \\
\text { Walton County }\end{array}$ & $\begin{array}{l}2,036 \\
(83 \%)\end{array}$ & $\begin{array}{l}4,620 \\
(81 \%)\end{array}$ & $\begin{array}{l}5,164 \\
(67 \%)\end{array}$ & $\begin{array}{l}2,526 \\
(36 \%)\end{array}$ & $\begin{array}{c}167 \\
(2 \%)\end{array}$ & $\begin{array}{c}14,513 \\
(49 \%)\end{array}$ \\
\hline $\begin{array}{l}\text { Deltona-Daytona Beach- } \\
\text { Ormond Beach (MSA) plus } \\
\text { Palm Coast (MSA) }\end{array}$ & $\begin{array}{l}8,162 \\
(68 \%)\end{array}$ & $\begin{array}{l}9,430 \\
(80 \%)\end{array}$ & $\begin{array}{c}14,671 \\
(82 \%)\end{array}$ & $\begin{array}{l}5,077 \\
(38 \%)\end{array}$ & $\begin{array}{c}787 \\
(4 \%)\end{array}$ & $\begin{array}{c}38,127 \\
(52 \%)\end{array}$ \\
\hline Fort Lauderdale & $\begin{array}{c}33,216 \\
(80 \%)\end{array}$ & $\begin{array}{c}38,276 \\
(93 \%)\end{array}$ & $\begin{array}{c}43,551 \\
(84 \%)\end{array}$ & $\begin{array}{c}24,871 \\
(45 \%)\end{array}$ & $\begin{array}{l}9,955 \\
(14 \%)\end{array}$ & $\begin{array}{c}149,869 \\
(58 \%)\end{array}$ \\
\hline $\begin{array}{l}\text { Gainesville (MSA) minus } \\
\text { Gilchrist County }\end{array}$ & $\begin{array}{l}10,359 \\
(70 \%)\end{array}$ & $\begin{array}{l}5,879 \\
(85 \%)\end{array}$ & $\begin{array}{l}4,342 \\
(56 \%) \\
\end{array}$ & $\begin{array}{c}912 \\
(13 \%)\end{array}$ & $\begin{array}{l}263 \\
(3 \%)\end{array}$ & $\begin{array}{l}21,755 \\
(49 \%)\end{array}$ \\
\hline Homosassa Springs (MSA) & $\begin{array}{l}1,063 \\
(95 \%)\end{array}$ & $\begin{array}{l}2,171 \\
(93 \%)\end{array}$ & $\begin{array}{l}1,831 \\
(58 \%)\end{array}$ & $\begin{array}{c}617 \\
(32 \%)\end{array}$ & $\begin{array}{c}78 \\
(3 \%)\end{array}$ & $\begin{array}{l}5,760 \\
(52 \%)\end{array}$ \\
\hline
\end{tabular}




\begin{tabular}{|c|c|c|c|c|c|c|}
\hline $\begin{array}{l}\text { Jacksonville (MSA) plus } \\
\text { Palatka ( } \mu S A)\end{array}$ & $\begin{array}{l}33,637 \\
(75 \%)\end{array}$ & $\begin{array}{l}26,633 \\
(86 \%)\end{array}$ & $\begin{array}{c}25,756 \\
(56 \%)\end{array}$ & $\begin{array}{l}7,180 \\
(19 \%)\end{array}$ & $\begin{array}{c}2,658 \\
(6 \%)\end{array}$ & $\begin{array}{c}95,864 \\
(47 \%)\end{array}$ \\
\hline $\begin{array}{l}\text { Lake City ( } \mu \text { SA) plus } \\
\text { Bradford, Dixie, Gilchrist, } \\
\text { Hamilton, Lafayette, Levy, } \\
\text { Madison, Suwannee, Taylor, } \\
\text { and Union Counties }\end{array}$ & $\begin{array}{l}3,687 \\
(52 \%)\end{array}$ & $\begin{array}{l}2,843 \\
(62 \%)\end{array}$ & $\begin{array}{l}1,798 \\
(46 \%)\end{array}$ & $\begin{array}{l}312 \\
(5 \%)\end{array}$ & $\begin{array}{c}0 \\
(0 \%)\end{array}$ & $\begin{array}{l}8,640 \\
(31 \%)\end{array}$ \\
\hline $\begin{array}{l}\text { Lakeland-Winter Haven } \\
\text { (MSA) }\end{array}$ & $\begin{array}{l}9,205 \\
(75 \%)\end{array}$ & $\begin{array}{l}9,306 \\
(84 \%)\end{array}$ & $\begin{array}{l}12,885 \\
(68 \%)\end{array}$ & $\begin{array}{l}5,385 \\
(37 \%)\end{array}$ & $\begin{array}{l}1,873 \\
(10 \%)\end{array}$ & $\begin{array}{c}38,654 \\
(51 \%)\end{array}$ \\
\hline $\begin{array}{l}\text { Miami-Dade plus Key West } \\
(\mu \mathrm{SA})\end{array}$ & $\begin{array}{c}54,666 \\
(66 \%)\end{array}$ & $\begin{array}{l}59,453 \\
(85 \%)\end{array}$ & $\begin{array}{c}70,684 \\
(85 \%)\end{array}$ & $\begin{array}{r}49,537 \\
(66 \%)\end{array}$ & $\begin{array}{r}23,950 \\
(19 \%)\end{array}$ & $\begin{array}{c}258,290 \\
(59 \%)\end{array}$ \\
\hline $\begin{array}{l}\text { Naples-Immokalee-Marco } \\
\text { Island (MSA) }\end{array}$ & $\begin{array}{l}3,864 \\
(89 \%)\end{array}$ & $\begin{array}{l}5,851 \\
(87 \%)\end{array}$ & $\begin{array}{l}5,380 \\
(58 \%)\end{array}$ & $\begin{array}{l}2,941 \\
(38 \%)\end{array}$ & $\begin{array}{c}252 \\
(3 \%)\end{array}$ & $\begin{array}{c}18,288 \\
(49 \%)\end{array}$ \\
\hline $\begin{array}{l}\text { North Port-Sarasota- } \\
\text { Bradenton (MSA) }\end{array}$ & $\begin{array}{l}9,796 \\
(75 \%)\end{array}$ & $\begin{array}{l}13,737 \\
(83 \%)\end{array}$ & $\begin{array}{c}12,981 \\
(62 \%)\end{array}$ & $\begin{array}{l}5,711 \\
(29 \%)\end{array}$ & $\begin{array}{l}1,758 \\
(8 \%)\end{array}$ & $\begin{array}{l}43,983 \\
(47 \%)\end{array}$ \\
\hline Ocala (MSA) & $\begin{array}{l}4,702 \\
(76 \%)\end{array}$ & $\begin{array}{l}3,167 \\
(75 \%)\end{array}$ & $\begin{array}{l}3,905 \\
(56 \%)\end{array}$ & $\begin{array}{l}379 \\
(7 \%)\end{array}$ & $\begin{array}{l}269 \\
(4 \%)\end{array}$ & $\begin{array}{c}12,422 \\
(42 \%)\end{array}$ \\
\hline $\begin{array}{l}\text { Orlando-Kissimmee-Sanford } \\
\text { (MSA) plus The Villages } \\
\text { (MSA) }\end{array}$ & $\begin{array}{c}40,870 \\
(78 \%)\end{array}$ & $\begin{array}{l}57,637 \\
(94 \%)\end{array}$ & $\begin{array}{c}57,209 \\
(75 \%)\end{array}$ & $\begin{array}{c}19,248 \\
(29 \%)\end{array}$ & $\begin{array}{c}3,525 \\
(4 \%)\end{array}$ & $\begin{array}{c}178,489 \\
(52 \%)\end{array}$ \\
\hline $\begin{array}{l}\text { Palm Bay-Melbourne- } \\
\text { Titusville (MSA) }\end{array}$ & $\begin{array}{l}7,840 \\
(69 \%)\end{array}$ & $\begin{array}{l}9,418 \\
(79 \%)\end{array}$ & $\begin{array}{l}8,496 \\
(59 \%)\end{array}$ & $\begin{array}{l}4,570 \\
(31 \%)\end{array}$ & $\begin{array}{l}762 \\
(5 \%)\end{array}$ & $\begin{array}{l}31,086 \\
(46 \%)\end{array}$ \\
\hline $\begin{array}{l}\text { Panama City (MSA) plus } \\
\text { Calhoun, Franklin, Gadsden, } \\
\text { Holmes, Jackson, Jefferson, } \\
\text { Liberty, Wakulla, Walton, } \\
\text { and Washington Counties }\end{array}$ & $\begin{array}{l}8,657 \\
(72 \%)\end{array}$ & $\begin{array}{l}5,775 \\
(71 \%)\end{array}$ & $\begin{array}{l}6,444 \\
(56 \%)\end{array}$ & $\begin{array}{l}2,922 \\
(29 \%)\end{array}$ & $\begin{array}{l}980 \\
(7 \%)\end{array}$ & $\begin{array}{c}24,778 \\
(45 \%)\end{array}$ \\
\hline $\begin{array}{l}\text { Pensacola-Ferry Pass-Brent } \\
\text { (MSA) }\end{array}$ & $\begin{array}{l}7,197 \\
(70 \%)\end{array}$ & $\begin{array}{l}7,544 \\
(87 \%)\end{array}$ & $\begin{array}{l}7,984 \\
(57 \%)\end{array}$ & $\begin{array}{l}2,174 \\
(15 \%)\end{array}$ & $\begin{array}{l}531 \\
(3 \%) \\
\end{array}$ & $\begin{array}{l}25,430 \\
(41 \%) \\
\end{array}$ \\
\hline Port St. Lucie (MSA) & $\begin{array}{l}6,240 \\
(74 \%) \\
\end{array}$ & $\begin{array}{l}7,459 \\
(90 \%) \\
\end{array}$ & $\begin{array}{l}7,108 \\
(79 \%) \\
\end{array}$ & $\begin{array}{l}2,412 \\
(27 \%)\end{array}$ & $\begin{array}{l}638 \\
(5 \%) \\
\end{array}$ & $\begin{array}{r}23,857 \\
(49 \%) \\
\end{array}$ \\
\hline Punta Gorda (MSA) & $\begin{array}{l}2,005 \\
(75 \%)\end{array}$ & $\begin{array}{l}1,597 \\
(67 \%)\end{array}$ & $\begin{array}{l}4,850 \\
(79 \%)\end{array}$ & $\begin{array}{c}475 \\
(16 \%)\end{array}$ & $\begin{array}{c}58 \\
(2 \%)\end{array}$ & $\begin{array}{l}8,985 \\
(50 \%)\end{array}$ \\
\hline Sebastian-Vero Beach (MSA) & $\begin{array}{l}1,733 \\
(81 \%)\end{array}$ & $\begin{array}{l}1,830 \\
(81 \%)\end{array}$ & $\begin{array}{l}1,459 \\
(52 \%)\end{array}$ & $\begin{array}{l}220 \\
(9 \%)\end{array}$ & $\begin{array}{c}0 \\
0 \%\end{array}$ & $\begin{array}{l}5,242 \\
(43 \%)\end{array}$ \\
\hline $\begin{array}{l}\text { Sebring (MSA) plus Arcadia, } \\
\text { Clewiston, Okeechobee, and } \\
\text { Wauchula ( } \mu \text { SAs) and Glades } \\
\text { County }\end{array}$ & $\begin{array}{l}2,737 \\
(73 \%)\end{array}$ & $\begin{array}{l}3,677 \\
(89 \%)\end{array}$ & $\begin{array}{l}4,182 \\
(59 \%)\end{array}$ & $\begin{array}{l}1,512 \\
(26 \%)\end{array}$ & $\begin{array}{c}54 \\
(1 \%)\end{array}$ & $\begin{array}{c}12,162 \\
(48 \%)\end{array}$ \\
\hline $\begin{array}{l}\text { Tallahassee (MSA) minus } \\
\text { Gadsden, Jefferson, and } \\
\text { Wakulla Counties }\end{array}$ & $\begin{array}{c}14,005 \\
(89 \%)\end{array}$ & $\begin{array}{l}9,330 \\
(88 \%)\end{array}$ & $\begin{array}{l}6,678 \\
(58 \%)\end{array}$ & $\begin{array}{l}1,050 \\
(11 \%)\end{array}$ & $\begin{array}{c}0 \\
(0 \%)\end{array}$ & $\begin{array}{r}31,063 \\
(59 \%)\end{array}$ \\
\hline $\begin{array}{l}\text { Tampa-St. Petersburg- } \\
\text { Clearwater (MSA) }\end{array}$ & $\begin{array}{c}57,863 \\
(73 \%) \\
\end{array}$ & $\begin{array}{l}60,926 \\
(88 \%)\end{array}$ & $\begin{array}{c}57,022 \\
(66 \%)\end{array}$ & $\begin{array}{c}22,888 \\
(27 \%)\end{array}$ & $\begin{array}{c}6,223 \\
(5 \%)\end{array}$ & $\begin{array}{c}204,922 \\
(47 \%)\end{array}$ \\
\hline $\begin{array}{l}\text { West Palm Beach-Boca } \\
\text { Raton }\end{array}$ & $\begin{array}{r}28,957 \\
(77 \%)\end{array}$ & $\begin{array}{l}25,966 \\
(88 \%)\end{array}$ & $\begin{array}{l}26,514 \\
(70 \%)\end{array}$ & $\begin{array}{c}11,270 \\
(37 \%)\end{array}$ & $\begin{array}{c}2,720 \\
(6 \%)\end{array}$ & $\begin{array}{c}95,427 \\
(53 \%)\end{array}$ \\
\hline
\end{tabular}


Number (and Percent) of Georgia Renter Households That Are Cost Burdened (Rent Is $>\mathbf{3 0}$ \% Household Income) by Income Category and by Combined PUMA Region

\begin{tabular}{|c|c|c|c|c|c|c|}
\hline & $\begin{array}{l}\text { Extremely } \\
\text { Low } \\
\text { Income } \\
\text { (30\% AMI } \\
\text { or Less) }\end{array}$ & $\begin{array}{l}\text { Very Low } \\
\text { Income } \\
\text { (30.01 to } \\
50 \% \text { AMI) }\end{array}$ & $\begin{array}{l}\text { Low } \\
\text { Income } \\
\text { (50.01 to } \\
80 \% \mathrm{AMI})\end{array}$ & $\begin{array}{l}\text { Moderate } \\
\text { Income } \\
\text { (80.01 to } \\
120 \% \text { AMI) }\end{array}$ & $\begin{array}{l}\text { Upper } \\
\text { Income } \\
\text { (More than } \\
\text { 120\% AMI) }\end{array}$ & $\begin{array}{c}\text { All Renter } \\
\text { House- } \\
\text { holds }\end{array}$ \\
\hline $\begin{array}{l}\text { Albany (MSA) plus } \\
\text { Bainbridge, Moultrie, and } \\
\text { Thomasville ( } \mu \text { SAs) and } \\
\text { Calhoun, Early, Grady, } \\
\text { Miller, Mitchell, and } \\
\text { Seminole Counties }\end{array}$ & $\begin{array}{l}8,584 \\
(66 \%)\end{array}$ & $\begin{array}{l}7,111 \\
(86 \%)\end{array}$ & $\begin{array}{l}5,647 \\
(64 \%)\end{array}$ & $\begin{array}{l}1,516 \\
(15 \%)\end{array}$ & $\begin{array}{l}127 \\
(1 \%)\end{array}$ & $\begin{array}{c}22,985 \\
(44 \%)\end{array}$ \\
\hline $\begin{array}{l}\text { Athens-Clarke County (MSA) } \\
\text { plus Elbert and Greene } \\
\text { Counties }\end{array}$ & $\begin{array}{l}9,565 \\
(72 \%)\end{array}$ & $\begin{array}{l}5,430 \\
(80 \%)\end{array}$ & $\begin{array}{l}4,571 \\
(65 \%)\end{array}$ & $\begin{array}{l}1,608 \\
(25 \%)\end{array}$ & $\begin{array}{l}212 \\
(3 \%)\end{array}$ & $\begin{array}{c}21,386 \\
(53 \%)\end{array}$ \\
\hline $\begin{array}{l}\text { Atlanta-Sandy Springs- } \\
\text { Roswell (MSA) minus } \\
\text { Haralson and Pickens } \\
\text { Counties plus Jefferson, } \\
\text { LaGrange, and Thomaston } \\
(\mu S A s)\end{array}$ & $\begin{array}{c}128,776 \\
(77 \%)\end{array}$ & $\begin{array}{c}116,379 \\
(86 \%)\end{array}$ & $\begin{array}{c}81,643 \\
(51 \%)\end{array}$ & $\begin{array}{c}20,166 \\
(13 \%)\end{array}$ & $\begin{array}{c}4,165 \\
(2 \%)\end{array}$ & $\begin{array}{c}351,129 \\
(45 \%)\end{array}$ \\
\hline $\begin{array}{l}\text { Augusta-Richmond County, } \\
\text { GA-SC (MSA) plus Glascock, } \\
\text { Jefferson, Jenkins, } \\
\text { Taliaferro, Warren, } \\
\text { Washington, and Wilkes } \\
\text { Counties }\end{array}$ & $\begin{array}{r}11,350 \\
(75 \%)\end{array}$ & $\begin{array}{l}8,243 \\
(82 \%)\end{array}$ & $\begin{array}{l}4,949 \\
(52 \%)\end{array}$ & $\begin{array}{c}798 \\
(8 \%)\end{array}$ & $\begin{array}{l}474 \\
(4 \%)\end{array}$ & $\begin{array}{c}25,814 \\
(45 \%)\end{array}$ \\
\hline $\begin{array}{l}\text { Brunswick (MSA) minus } \\
\text { Brantley County plus St. } \\
\text { Marys ( } \mu \mathrm{SA} \text { ) }\end{array}$ & $\begin{array}{l}3,392 \\
(73 \%)\end{array}$ & $\begin{array}{l}3,983 \\
(83 \%)\end{array}$ & $\begin{array}{l}2,675 \\
(75 \%)\end{array}$ & $\begin{array}{l}1,014 \\
(20 \%)\end{array}$ & $\begin{array}{c}0 \\
(0 \%)\end{array}$ & $\begin{array}{c}11,064 \\
(46 \%)\end{array}$ \\
\hline $\begin{array}{l}\text { Chattanooga, TN-GA (MSA) } \\
\text { plus Summerville }(\mu S A)\end{array}$ & $\begin{array}{l}2,944 \\
(83 \%) \\
\end{array}$ & $\begin{array}{l}2,550 \\
(60 \%) \\
\end{array}$ & $\begin{array}{l}1,136 \\
(42 \%) \\
\end{array}$ & $\begin{array}{c}0 \\
(0 \%) \\
\end{array}$ & $\begin{array}{c}0 \\
0 \% \\
(0 \%) \\
\end{array}$ & $\begin{array}{l}6,630 \\
(38 \%) \\
\end{array}$ \\
\hline $\begin{array}{l}\text { Columbus, GA-AL (MSA) plus } \\
\text { Americus, Eufaula, AL-GA, } \\
\text { and Cordele ( } \mu \text { SAs) and Clay, } \\
\text { Dooley, Macon, Randolph, } \\
\text { Stewart, Talbot, Taylor, and } \\
\text { Webster Counties }\end{array}$ & $\begin{array}{c}10,529 \\
(75 \%)\end{array}$ & $\begin{array}{l}7,194 \\
(69 \%)\end{array}$ & $\begin{array}{l}7,198 \\
(65 \%)\end{array}$ & $\begin{array}{l}3,118 \\
(28 \%)\end{array}$ & $\begin{array}{l}746 \\
(5 \%)\end{array}$ & $\begin{array}{c}28,785 \\
(47 \%)\end{array}$ \\
\hline $\begin{array}{l}\text { Cornelia ( } \mu \mathrm{SA}) \text { plus Toccoa } \\
(\mu \mathrm{SA}) \text { plus Banks, Franklin, } \\
\text { and Hart Counties }\end{array}$ & $\begin{array}{l}2,354 \\
(64 \%)\end{array}$ & $\begin{array}{l}1,460 \\
(59 \%)\end{array}$ & $\begin{array}{l}1,588 \\
(58 \%)\end{array}$ & $\begin{array}{c}99 \\
(5 \%)\end{array}$ & $\begin{array}{c}0 \\
(0 \%)\end{array}$ & $\begin{array}{l}5,501 \\
(37 \%)\end{array}$ \\
\hline
\end{tabular}




\begin{tabular}{|c|c|c|c|c|c|c|}
\hline $\begin{array}{l}\text { Dalton (MSA) plus Calhoun } \\
(\mu \mathrm{SA}) \text { and Fannin, Gilmer, } \\
\text { and Pickens Counties }\end{array}$ & $\begin{array}{l}3,099 \\
(63 \%)\end{array}$ & $\begin{array}{l}5,203 \\
(80 \%)\end{array}$ & $\begin{array}{l}2,450 \\
(27 \%)\end{array}$ & $\begin{array}{c}262 \\
(4 \%)\end{array}$ & $\begin{array}{c}0 \\
(0 \%)\end{array}$ & $\begin{array}{l}11,014 \\
(36 \%)\end{array}$ \\
\hline $\begin{array}{l}\text { Dublin, GA ( } \mu \mathrm{SA} \text { ) plus } \\
\text { Bleckley, Candler, Dodge, } \\
\text { Emanuel, Treutlen, and } \\
\text { Wilcox Counties }\end{array}$ & $\begin{array}{l}1,936 \\
(50 \%)\end{array}$ & $\begin{array}{l}1,934 \\
(63 \%)\end{array}$ & $\begin{array}{l}1,144 \\
(42 \%)\end{array}$ & $\begin{array}{c}259 \\
(12 \%)\end{array}$ & $\begin{array}{c}0 \\
(0 \%)\end{array}$ & $\begin{array}{l}5,273 \\
(34 \%)\end{array}$ \\
\hline Gainesville (MSA) & $\begin{array}{l}3,032 \\
(65 \%)\end{array}$ & $\begin{array}{l}3,090 \\
(70 \%)\end{array}$ & $\begin{array}{l}1,703 \\
(50 \%)\end{array}$ & $\begin{array}{c}832 \\
(16 \%)\end{array}$ & $\begin{array}{l}189 \\
(4 \%)\end{array}$ & $\begin{array}{l}8,846 \\
(40 \%)\end{array}$ \\
\hline $\begin{array}{l}\text { Hinesville (MSA) plus Bryan } \\
\text { County }\end{array}$ & $\begin{array}{l}2,870 \\
(77 \%)\end{array}$ & $\begin{array}{l}1,446 \\
(88 \%)\end{array}$ & $\begin{array}{l}3,929 \\
(80 \%)\end{array}$ & $\begin{array}{c}942 \\
(44 \%)\end{array}$ & $\begin{array}{c}809 \\
(14 \%)\end{array}$ & $\begin{array}{l}9,996 \\
(56 \%)\end{array}$ \\
\hline $\begin{array}{l}\text { Macon-Bibb County (MSA) } \\
\text { plus Warner Robins (MSA) } \\
\text { and Baldwin, Putnam, and } \\
\text { Wilkinson Counties }\end{array}$ & $\begin{array}{r}13,050 \\
(61 \%)\end{array}$ & $\begin{array}{l}10,320 \\
(85 \%)\end{array}$ & $\begin{array}{l}6,976 \\
(61 \%)\end{array}$ & $\begin{array}{l}1,519 \\
(15 \%)\end{array}$ & $\begin{array}{l}332 \\
(3 \%)\end{array}$ & $\begin{array}{r}32,197 \\
(48 \%)\end{array}$ \\
\hline $\begin{array}{l}\text { Northeast Georgia } \\
\text { Nonmetropolitan Area }\end{array}$ & $\begin{array}{l}1,836 \\
(65 \%) \\
\end{array}$ & $\begin{array}{l}1,373 \\
(54 \%) \\
\end{array}$ & $\begin{array}{l}1,305 \\
(51 \%) \\
\end{array}$ & $\begin{array}{c}252 \\
(10 \%) \\
\end{array}$ & $\begin{array}{c}64 \\
(3 \%) \\
\end{array}$ & $\begin{array}{l}4,830 \\
(39 \%) \\
\end{array}$ \\
\hline $\begin{array}{l}\text { Rome (MSA) plus } \\
\text { Cedartown }(\mu S A) \text { plus } \\
\text { Haralson County }\end{array}$ & $\begin{array}{l}4,654 \\
(90 \%)\end{array}$ & $\begin{array}{l}3,304 \\
(71 \%)\end{array}$ & $\begin{array}{l}3,134 \\
(52 \%)\end{array}$ & $\begin{array}{c}13 \\
(0 \%)\end{array}$ & $\begin{array}{c}58 \\
(1 \%)\end{array}$ & $\begin{array}{l}11,163 \\
(46 \%)\end{array}$ \\
\hline $\begin{array}{l}\text { Savannah (MSA) minus } \\
\text { Bryan County plus } \\
\text { Statesboro }(\mu \mathrm{SA}) \text { and } \\
\text { Screven County }\end{array}$ & $\begin{array}{c}11,519 \\
(71 \%)\end{array}$ & $\begin{array}{l}10,043 \\
(81 \%)\end{array}$ & $\begin{array}{c}11,391 \\
(72 \%)\end{array}$ & $\begin{array}{l}2,411 \\
(19 \%)\end{array}$ & $\begin{array}{c}397 \\
(2 \%)\end{array}$ & $\begin{array}{c}35,761 \\
(48 \%)\end{array}$ \\
\hline $\begin{array}{l}\text { Valdosta (MSA) plus } \\
\text { Fitzgerald, Douglas, Tifton, } \\
\text { and Waycross ( } \mu \text { SAs) and } \\
\text { Atkinson, Bacon, Berrien, } \\
\text { Brantley, Charlton, Clinch, } \\
\text { Cook, Irwin, and Turner } \\
\text { Counties }\end{array}$ & $\begin{array}{l}6,130 \\
(57 \%)\end{array}$ & $\begin{array}{l}8,462 \\
(81 \%)\end{array}$ & $\begin{array}{l}6,519 \\
(58 \%)\end{array}$ & $\begin{array}{l}2,199 \\
(24 \%)\end{array}$ & $\begin{array}{c}80 \\
(1 \%)\end{array}$ & $\begin{array}{c}23,390 \\
(44 \%)\end{array}$ \\
\hline $\begin{array}{l}\text { Vidalia ( } \mu S A) \text { plus Jesup } \\
(\mu S A) \text { and Appling, Evans, } \\
\text { Jeff Davis, Tattnall, Telfair, } \\
\text { and Wheeler Counties }\end{array}$ & $\begin{array}{l}2,373 \\
(55 \%)\end{array}$ & $\begin{array}{l}1,160 \\
(47 \%)\end{array}$ & $\begin{array}{l}1,340 \\
(36 \%)\end{array}$ & $\begin{array}{c}143 \\
(4 \%)\end{array}$ & $\begin{array}{c}0 \\
(0 \%)\end{array}$ & $\begin{array}{l}5,016 \\
(28 \%)\end{array}$ \\
\hline
\end{tabular}


Number (and Percent) of Louisiana Renter Households That Ae Cost Burdened (Rent Is >30\% Household Income) by Income Category and by Combined PUMA Region

\begin{tabular}{|c|c|c|c|c|c|c|}
\hline & $\begin{array}{l}\text { Extremely } \\
\text { Low } \\
\text { Income } \\
\text { (30\% AMI } \\
\text { or Less) }\end{array}$ & $\begin{array}{l}\text { Very Low } \\
\text { Income } \\
\text { (30.01 to } \\
50 \% \text { AMI) }\end{array}$ & $\begin{array}{l}\text { Low } \\
\text { Income } \\
\text { (50.01 to } \\
80 \% \text { AMI) }\end{array}$ & $\begin{array}{l}\text { Moderate } \\
\text { Income } \\
\text { (80.01 to } \\
120 \% \text { AMI) }\end{array}$ & $\begin{array}{c}\text { Upper } \\
\text { Income } \\
\text { (More } \\
\text { than } \\
120 \% \\
\text { AMI) }\end{array}$ & $\begin{array}{c}\text { All Renter } \\
\text { House- } \\
\text { holds }\end{array}$ \\
\hline $\begin{array}{l}\text { Alexandria (MSA) plus Fort } \\
\text { Polk South ( } \mu S A) \text { and } \\
\text { Natchez, MS-LA ( } \mu S A) \text { and } \\
\text { Avoyelles, Catahoula, } \\
\text { LaSalle, and Winn Parishes }\end{array}$ & $\begin{array}{l}5,466 \\
(64 \%)\end{array}$ & $\begin{array}{l}7,134 \\
(83 \%)\end{array}$ & $\begin{array}{l}3,100 \\
(39 \%)\end{array}$ & $\begin{array}{l}1,305 \\
(16 \%)\end{array}$ & $\begin{array}{l}137 \\
(1 \%)\end{array}$ & $\begin{array}{c}17,142 \\
(40 \%)\end{array}$ \\
\hline Baton Rouge (MSA) & $\begin{array}{c}18,954 \\
(73 \%)\end{array}$ & $\begin{array}{c}14,169 \\
(73 \%)\end{array}$ & $\begin{array}{l}7,224 \\
(40 \%)\end{array}$ & $\begin{array}{l}1,333 \\
(9 \%)\end{array}$ & $\begin{array}{l}531 \\
(4 \%)\end{array}$ & $\begin{array}{l}42,211 \\
(46 \%)\end{array}$ \\
\hline $\begin{array}{l}\text { Hammond (MSA) plus } \\
\text { Bogalusa }(\mu \mathrm{SA})\end{array}$ & $\begin{array}{l}5,648 \\
(68 \%)\end{array}$ & $\begin{array}{l}5,117 \\
(87 \%)\end{array}$ & $\begin{array}{c}536 \\
(25 \%)\end{array}$ & $\begin{array}{c}90 \\
(3 \%)\end{array}$ & $\begin{array}{c}0 \\
0 \%\end{array}$ & $\begin{array}{c}11,391 \\
(48 \%)\end{array}$ \\
\hline $\begin{array}{l}\text { Houma-Thibodaux (MSA) } \\
\text { plus Assumption Parish }\end{array}$ & $\begin{array}{l}2,252 \\
(66 \%) \\
\end{array}$ & $\begin{array}{l}4,140 \\
(74 \%) \\
\end{array}$ & $\begin{array}{l}2,292 \\
(48 \%) \\
\end{array}$ & $\begin{array}{c}427 \\
(14 \%)\end{array}$ & $\begin{array}{c}0 \\
(0 \%)\end{array}$ & $\begin{array}{l}9,111 \\
(41 \%)\end{array}$ \\
\hline $\begin{array}{l}\text { Lafayette (MSA) plus } \\
\text { Morgan City }(\mu S A)\end{array}$ & $\begin{array}{c}11,943 \\
(70 \%)\end{array}$ & $\begin{array}{l}9,607 \\
(76 \%)\end{array}$ & $\begin{array}{l}2,742 \\
(26 \%)\end{array}$ & $\begin{array}{c}914 \\
(8 \%)\end{array}$ & $\begin{array}{c}80 \\
(1 \%)\end{array}$ & $\begin{array}{c}24,996 \\
(40 \%)\end{array}$ \\
\hline $\begin{array}{l}\text { Lake Charles (MSA) plus } \\
\text { DeRidder }(\mu \mathrm{SA}) \text { and Jennings } \\
(\mu \mathrm{SA}) \text { and Allen Parish }\end{array}$ & $\begin{array}{l}5,184 \\
(76 \%)\end{array}$ & $\begin{array}{l}3,824 \\
(62 \%)\end{array}$ & $\begin{array}{l}3,674 \\
(44 \%)\end{array}$ & $\begin{array}{c}533 \\
(10 \%)\end{array}$ & $\begin{array}{c}0 \\
(0 \%)\end{array}$ & $\begin{array}{l}13,215 \\
(38 \%)\end{array}$ \\
\hline $\begin{array}{l}\text { Monroe (MSA) plus Bastrop } \\
(\mu \mathrm{SA}) \text { and Caldwell, East } \\
\text { Carroll, Franklin, Jackson, } \\
\text { Madison, Richland, Tensas, } \\
\text { and West Carroll Parishes }\end{array}$ & $\begin{array}{l}7,837 \\
(60 \%)\end{array}$ & $\begin{array}{l}5,662 \\
(74 \%)\end{array}$ & $\begin{array}{l}3,871 \\
(39 \%)\end{array}$ & $\begin{array}{c}621 \\
(12 \%)\end{array}$ & $\begin{array}{c}0 \\
(0 \%)\end{array}$ & $\begin{array}{c}17,991 \\
(43 \%)\end{array}$ \\
\hline $\begin{array}{l}\text { New Orleans-Metairie } \\
\text { (MSA) }\end{array}$ & $\begin{array}{c}36,839 \\
(72 \%)\end{array}$ & $\begin{array}{c}27,994 \\
(87 \%)\end{array}$ & $\begin{array}{c}22,284 \\
(61 \%)\end{array}$ & $\begin{array}{l}7,802 \\
(25 \%)\end{array}$ & $\begin{array}{c}1,764 \\
(4 \%)\end{array}$ & $\begin{array}{l}96,683 \\
(50 \%) \\
\end{array}$ \\
\hline $\begin{array}{l}\text { Opelousas ( } \mu \mathrm{SA}) \text { plus } \\
\text { Evangeline Parish }\end{array}$ & $\begin{array}{l}1,524 \\
(58 \%)\end{array}$ & $\begin{array}{l}2,624 \\
(74 \%) \\
\end{array}$ & $\begin{array}{l}1,603 \\
(73 \%) \\
\end{array}$ & $\begin{array}{c}101 \\
(5 \%)\end{array}$ & $\begin{array}{c}0 \\
(0 \%) \\
\end{array}$ & $\begin{array}{l}5,852 \\
(43 \%) \\
\end{array}$ \\
\hline $\begin{array}{l}\text { Ruston ( } \mu \mathrm{SA} \text { ) plus } \\
\text { Natchitoches ( } \mu \mathrm{SA} \text { ) and } \\
\text { DeSoto, Bienville, Claiborne, } \\
\text { Red River, and Sabine } \\
\text { Parishes }\end{array}$ & $\begin{array}{l}5,360 \\
(62 \%)\end{array}$ & $\begin{array}{l}3,219 \\
(66 \%)\end{array}$ & $\begin{array}{l}2,344 \\
(42 \%)\end{array}$ & $\begin{array}{c}655 \\
(16 \%)\end{array}$ & $\begin{array}{c}0 \\
(0 \%)\end{array}$ & $\begin{array}{c}11,578 \\
(45 \%)\end{array}$ \\
\hline $\begin{array}{l}\text { Shreveport-Bossier City } \\
\text { (MSA) minus DeSoto Parish }\end{array}$ & $\begin{array}{c}11,471 \\
(68 \%)\end{array}$ & $\begin{array}{l}8,679 \\
(83 \%) \\
\end{array}$ & $\begin{array}{l}8,093 \\
(59 \%) \\
\end{array}$ & $\begin{array}{l}1,779 \\
(18 \%)\end{array}$ & $\begin{array}{l}807 \\
(7 \%) \\
\end{array}$ & $\begin{array}{c}30,829 \\
(49 \%) \\
\end{array}$ \\
\hline
\end{tabular}


Number (and Percent) of Mississippi Renter Households That Are Cost Burdened (Rent Is $>\mathbf{3 0} \%$ Household Income) by Income Category and by Combined PUMA Region

\begin{tabular}{|c|c|c|c|c|c|c|}
\hline & $\begin{array}{l}\text { Extremely } \\
\text { Low } \\
\text { Income } \\
\text { (30\% AMI } \\
\text { or Less) }\end{array}$ & $\begin{array}{l}\text { Very Low } \\
\text { Income } \\
\text { (30.01 to } \\
50 \% \text { AMI) }\end{array}$ & $\begin{array}{l}\text { Low } \\
\text { Income } \\
\text { (50.01 to } \\
80 \% \text { AMI) }\end{array}$ & $\begin{array}{l}\text { Moderate } \\
\text { Income } \\
\text { (80.01 to } \\
120 \% \text { AMI) }\end{array}$ & $\begin{array}{c}\text { Upper } \\
\text { Income } \\
\text { (More } \\
\text { than } \\
120 \% \\
\text { AMI) }\end{array}$ & $\begin{array}{l}\text { All Renter } \\
\text { House- } \\
\text { holds }\end{array}$ \\
\hline $\begin{array}{l}\text { Brookhaven ( } \mu S A) \text { plus } \\
\text { McComb ( } \mu S A) \text { and } \\
\text { Vicksburg }(\mu S A)\end{array}$ & $\begin{array}{l}2,684 \\
(56 \%)\end{array}$ & $\begin{array}{l}2,115 \\
(61 \%)\end{array}$ & $\begin{array}{l}1,101 \\
(43 \%)\end{array}$ & $\begin{array}{c}282 \\
(6 \%)\end{array}$ & $\begin{array}{c}0 \\
(0 \%)\end{array}$ & $\begin{array}{l}6,182 \\
(34 \%)\end{array}$ \\
\hline $\begin{array}{l}\text { Cleveland }(\mu S A) \text { plus } \\
\text { Indianola }(\mu S A) \text { and } \\
\text { Greenville }(\mu S A)\end{array}$ & $\begin{array}{l}2,671 \\
(73 \%)\end{array}$ & $\begin{array}{l}3,188 \\
(79 \%)\end{array}$ & $\begin{array}{l}2,995 \\
(64 \%)\end{array}$ & $\begin{array}{l}1,988 \\
(59 \%)\end{array}$ & $\begin{array}{c}170 \\
(3 \%)\end{array}$ & $\begin{array}{c}11,012 \\
(53 \%)\end{array}$ \\
\hline $\begin{array}{l}\text { Columbus ( } \mu \mathrm{SA}) \text { plus } \\
\text { Starkville }(\mu \mathrm{SA}) \text { and West } \\
\text { Point }(\mu \mathrm{SA})\end{array}$ & $\begin{array}{l}3,633 \\
(46 \%)\end{array}$ & $\begin{array}{l}2,010 \\
(71 \%)\end{array}$ & $\begin{array}{l}2,987 \\
(65 \%)\end{array}$ & $\begin{array}{c}474 \\
(13 \%)\end{array}$ & $\begin{array}{c}105 \\
(3 \%)\end{array}$ & $\begin{array}{l}9,209 \\
(41 \%)\end{array}$ \\
\hline $\begin{array}{l}\text { Greenwood }(\mu S A) \text { plus } \\
\text { Grenada }(\mu S A)\end{array}$ & $\begin{array}{l}2,497 \\
(48 \%) \\
\end{array}$ & $\begin{array}{l}1,556 \\
(56 \%) \\
\end{array}$ & $\begin{array}{c}873 \\
(43 \%) \\
\end{array}$ & $\begin{array}{r}237 \\
(8 \%) \\
\end{array}$ & $\begin{array}{c}0 \\
(0 \%) \\
\end{array}$ & $\begin{array}{l}5,163 \\
(30 \%) \\
\end{array}$ \\
\hline $\begin{array}{l}\text { Gulfport-Biloxi-Pascagoula } \\
\text { (MSA) plus Picayune ( } \mu \text { SA) } \\
\text { and George and Stone } \\
\text { Counties }\end{array}$ & $\begin{array}{l}8,636 \\
(64 \%)\end{array}$ & $\begin{array}{l}8,701 \\
(78 \%)\end{array}$ & $\begin{array}{l}5,641 \\
(54 \%)\end{array}$ & $\begin{array}{l}1,639 \\
(13 \%)\end{array}$ & $\begin{array}{c}699 \\
(4 \%)\end{array}$ & $\begin{array}{c}25,316 \\
(40 \%)\end{array}$ \\
\hline $\begin{array}{l}\text { Hattiesburg (MSA) plus } \\
\text { Marion County }\end{array}$ & $\begin{array}{l}3,154 \\
(63 \%)\end{array}$ & $\begin{array}{l}4,696 \\
(74 \%)\end{array}$ & $\begin{array}{l}2,659 \\
(55 \%)\end{array}$ & $\begin{array}{c}397 \\
(15 \%)\end{array}$ & $\begin{array}{c}0 \\
(0 \%)\end{array}$ & $\begin{array}{c}10,906 \\
(47 \%)\end{array}$ \\
\hline Jackson (MSA) & $\begin{array}{c}13,599 \\
(67 \%)\end{array}$ & $\begin{array}{l}9,331 \\
(66 \%)\end{array}$ & $\begin{array}{l}9,005 \\
(57 \%)\end{array}$ & $\begin{array}{l}1,847 \\
(17 \%)\end{array}$ & $\begin{array}{l}466 \\
(3 \%)\end{array}$ & $\begin{array}{c}34,248 \\
(45 \%)\end{array}$ \\
\hline Laurel $(\mu \mathrm{SA})$ & $\begin{array}{l}1,558 \\
(61 \%)\end{array}$ & $\begin{array}{l}1,351 \\
(63 \%)\end{array}$ & $\begin{array}{l}1,760 \\
(65 \%)\end{array}$ & $\begin{array}{c}515 \\
(22 \%)\end{array}$ & $\begin{array}{c}0 \\
(0 \%)\end{array}$ & $\begin{array}{l}5,184 \\
(43 \%)\end{array}$ \\
\hline Memphis, TN-MS-AR (MSA) & $\begin{array}{l}7,455 \\
(67 \%)\end{array}$ & $\begin{array}{l}5,184 \\
(67 \%)\end{array}$ & $\begin{array}{l}3,837 \\
(40 \%)\end{array}$ & $\begin{array}{c}930 \\
(12 \%)\end{array}$ & $\begin{array}{l}233 \\
(3 \%)\end{array}$ & $\begin{array}{c}17,639 \\
(40 \%)\end{array}$ \\
\hline $\begin{array}{l}\text { Meridian }(\mu \mathrm{SA}) \text { plus Laurel } \\
(\mu \mathrm{SA})\end{array}$ & $\begin{array}{l}2,498 \\
(54 \%)\end{array}$ & $\begin{array}{l}3,389 \\
(71 \%)\end{array}$ & $\begin{array}{l}3,112 \\
(58 \%)\end{array}$ & $\begin{array}{l}1,040 \\
(20 \%)\end{array}$ & $\begin{array}{l}283 \\
(5 \%)\end{array}$ & $\begin{array}{c}10,322 \\
(41 \%)\end{array}$ \\
\hline $\begin{array}{l}\text { Tupelo }(\mu S A) \text { plus Oxford } \\
(\mu S A)\end{array}$ & $\begin{array}{l}7,701 \\
(75 \%)\end{array}$ & $\begin{array}{l}4,366 \\
(72 \%)\end{array}$ & $\begin{array}{l}3,573 \\
(48 \%)\end{array}$ & $\begin{array}{l}1,924 \\
(24 \%)\end{array}$ & $\begin{array}{c}0 \\
(0 \%)\end{array}$ & $\begin{array}{c}17,564 \\
(46 \%)\end{array}$ \\
\hline
\end{tabular}




\section{Number (and Percent) of Tennessee Renter Households That Are Cost Burdened (Rent Is} $\mathbf{3 0}$ \% Household Income) by Income Category and by Combined PUMA Region

\begin{tabular}{|c|c|c|c|c|c|c|}
\hline & $\begin{array}{c}\text { Extremely } \\
\text { Low } \\
\text { Income } \\
\text { (30\% AMI } \\
\text { or Less) }\end{array}$ & $\begin{array}{l}\text { Very Low } \\
\text { Income } \\
\text { (30.01 to } \\
50 \% \text { AMI) }\end{array}$ & $\begin{array}{l}\text { Low } \\
\text { Income } \\
\text { (50.01 to } \\
80 \% \text { AMI) }\end{array}$ & $\begin{array}{l}\text { Moderate } \\
\text { Income } \\
\text { (80.01 to } \\
120 \% \text { AMI) }\end{array}$ & $\begin{array}{c}\text { Upper } \\
\text { Income } \\
\text { (More } \\
\text { than } \\
120 \% \\
\text { AMI) }\end{array}$ & $\begin{array}{c}\text { All Renter } \\
\text { House- } \\
\text { holds }\end{array}$ \\
\hline $\begin{array}{l}\text { Brownsville ( } \mu S A) \text { plus } \\
\text { Decatur, Hardeman, Hardin, } \\
\text { Henderson, and McNairy } \\
\text { Counties }\end{array}$ & $\begin{array}{l}1,701 \\
(55 \%)\end{array}$ & $\begin{array}{l}2,454 \\
(82 \%)\end{array}$ & $\begin{array}{c}963 \\
(37 \%)\end{array}$ & $\begin{array}{l}105 \\
(5 \%)\end{array}$ & $\begin{array}{c}0 \\
(0 \%)\end{array}$ & $\begin{array}{l}5,223 \\
(34 \%)\end{array}$ \\
\hline $\begin{array}{l}\text { Chattanooga, TN-GA (MSA) } \\
\text { plus Bledsoe, Grundy, } \\
\text { Meigs, and Rhea Counties }\end{array}$ & $\begin{array}{c}12,056 \\
(76 \%)\end{array}$ & $\begin{array}{l}6,802 \\
(59 \%)\end{array}$ & $\begin{array}{l}5,971 \\
(40 \%)\end{array}$ & $\begin{array}{c}601 \\
(5 \%)\end{array}$ & $\begin{array}{l}275 \\
(3 \%)\end{array}$ & $\begin{array}{r}25,705 \\
(41 \%)\end{array}$ \\
\hline $\begin{array}{l}\text { Clarksville, TN-KY (MSA) plus } \\
\text { Stewart County }\end{array}$ & $\begin{array}{l}3,059 \\
(69 \%)\end{array}$ & $\begin{array}{l}2,796 \\
(80 \%)\end{array}$ & $\begin{array}{l}5,996 \\
(64 \%)\end{array}$ & $\begin{array}{l}1,360 \\
(19 \%)\end{array}$ & $\begin{array}{l}306 \\
(4 \%)\end{array}$ & $\begin{array}{l}13,517 \\
(43 \%)\end{array}$ \\
\hline $\begin{array}{l}\text { Cleveland (MSA) plus } \\
\text { McMinn County }\end{array}$ & $\begin{array}{l}5,315 \\
(81 \%) \\
\end{array}$ & $\begin{array}{l}3,051 \\
(72 \%) \\
\end{array}$ & $\begin{array}{l}2,073 \\
(47 \%) \\
\end{array}$ & $\begin{array}{c}704 \\
(16 \%) \\
\end{array}$ & $\begin{array}{c}0 \\
(0 \%)\end{array}$ & $\begin{array}{l}11,143 \\
(48 \%) \\
\end{array}$ \\
\hline $\begin{array}{l}\text { Cookeville ( } \mu \text { SA) plus Clay } \\
\text { and Picket Counties }\end{array}$ & $\begin{array}{l}2,217 \\
(59 \%)\end{array}$ & $\begin{array}{l}1,748 \\
(70 \%)\end{array}$ & $\begin{array}{l}2,017 \\
(55 \%)\end{array}$ & $\begin{array}{l}176 \\
(7 \%)\end{array}$ & $\begin{array}{c}0 \\
(0 \%)\end{array}$ & $\begin{array}{l}6,158 \\
(41 \%)\end{array}$ \\
\hline $\begin{array}{l}\text { Crossville ( } \mu \mathrm{SA}) \text { plus } \\
\text { Fentress, Van Buren, and } \\
\text { White Counties }\end{array}$ & $\begin{array}{l}1,828 \\
(73 \%)\end{array}$ & $\begin{array}{c}633 \\
(50 \%)\end{array}$ & $\begin{array}{l}1,365 \\
(53 \%)\end{array}$ & $\begin{array}{l}135 \\
(8 \%)\end{array}$ & $\begin{array}{c}0 \\
(0 \%)\end{array}$ & $\begin{array}{l}3,961 \\
(41 \%)\end{array}$ \\
\hline $\begin{array}{l}\text { Jackson (MSA) plus Crockett, } \\
\text { Dyer, and Lake Counties }\end{array}$ & $\begin{array}{l}4,706 \\
(59 \%)\end{array}$ & $\begin{array}{l}3,946 \\
(68 \%)\end{array}$ & $\begin{array}{l}3,823 \\
(56 \%) \\
\end{array}$ & $\begin{array}{c}492 \\
(12 \%) \\
\end{array}$ & $\begin{array}{c}63 \\
(1 \%)\end{array}$ & $\begin{array}{c}13,030 \\
(41 \%) \\
\end{array}$ \\
\hline $\begin{array}{l}\text { Johnson City (MSA) plus } \\
\text { Johnson and Greene } \\
\text { Counties }\end{array}$ & $\begin{array}{l}6,732 \\
(73 \%)\end{array}$ & $\begin{array}{l}3,984 \\
(73 \%)\end{array}$ & $\begin{array}{l}3,527 \\
(39 \%)\end{array}$ & $\begin{array}{c}900 \\
(20 \%)\end{array}$ & $\begin{array}{l}492 \\
(7 \%)\end{array}$ & $\begin{array}{c}15,635 \\
(45 \%)\end{array}$ \\
\hline $\begin{array}{l}\text { Kingsport-Bristol-Bristol, TN- } \\
\text { VA (MSA) }\end{array}$ & $\begin{array}{l}3,926 \\
(60 \%)\end{array}$ & $\begin{array}{l}2,994 \\
(67 \%)\end{array}$ & $\begin{array}{l}1,585 \\
(38 \%)\end{array}$ & $\begin{array}{l}1,190 \\
(26 \%)\end{array}$ & $\begin{array}{l}222 \\
(5 \%)\end{array}$ & $\begin{array}{l}9,917 \\
(41 \%)\end{array}$ \\
\hline $\begin{array}{l}\text { Knoxville (MSA) plus } \\
\text { Claiborne, Hancock, } \\
\text { Monroe, and Scott Counties } \\
\text { minus Grainger County }\end{array}$ & $\begin{array}{c}23,559 \\
(70 \%)\end{array}$ & $\begin{array}{l}15,220 \\
(66 \%)\end{array}$ & $\begin{array}{l}9,061 \\
(35 \%)\end{array}$ & $\begin{array}{l}834 \\
(5 \%)\end{array}$ & $\begin{array}{l}520 \\
(3 \%)\end{array}$ & $\begin{array}{c}49,194 \\
(41 \%)\end{array}$ \\
\hline $\begin{array}{l}\text { Lawrenceburg ( } \mu \mathrm{SA} \text { ) plus } \\
\text { Giles, Lewis, Perry, and } \\
\text { Wayne Counties }\end{array}$ & $\begin{array}{l}1,717 \\
(79 \%)\end{array}$ & $\begin{array}{l}1,041 \\
(58 \%)\end{array}$ & $\begin{array}{l}1,012 \\
(37 \%)\end{array}$ & $\begin{array}{c}219 \\
(19 \%)\end{array}$ & $\begin{array}{c}0 \\
(0 \%)\end{array}$ & $\begin{array}{l}3,989 \\
(43 \%)\end{array}$ \\
\hline $\begin{array}{l}\text { Martin ( } \mu S A) \text { plus Paris } \\
(\mu S A) \text { and Benton, Carroll, } \\
\text { Houston, and Humphreys } \\
\text { Counties }\end{array}$ & $\begin{array}{l}2,719 \\
(81 \%)\end{array}$ & $\begin{array}{l}2,728 \\
(79 \%)\end{array}$ & $\begin{array}{c}865 \\
(24 \%)\end{array}$ & $\begin{array}{c}95 \\
(3 \%)\end{array}$ & $\begin{array}{c}59 \\
(3 \%)\end{array}$ & $\begin{array}{l}6,466 \\
(42 \%)\end{array}$ \\
\hline $\begin{array}{l}\text { Memphis, TN-MS-AR (MSA) } \\
\text { plus Lauderdale County }\end{array}$ & $\begin{array}{c}31,266 \\
(74 \%) \\
\end{array}$ & $\begin{array}{c}24,151 \\
(80 \%) \\
\end{array}$ & $\begin{array}{c}17,317 \\
(48 \%) \\
\end{array}$ & $\begin{array}{l}3,974 \\
(15 \%) \\
\end{array}$ & $\begin{array}{l}965 \\
(3 \%) \\
\end{array}$ & $\begin{array}{c}77,673 \\
(46 \%) \\
\end{array}$ \\
\hline
\end{tabular}




\begin{tabular}{|c|c|c|c|c|c|c|}
\hline $\begin{array}{l}\text { Morristown (MSA) plus } \\
\text { Cocke, Grainger, and Sevier } \\
\text { Counties }\end{array}$ & $\begin{array}{l}4,089 \\
(52 \%)\end{array}$ & $\begin{array}{l}3,555 \\
(62 \%)\end{array}$ & $\begin{array}{l}4,477 \\
(51 \%)\end{array}$ & $\begin{array}{l}1,166 \\
(20 \%)\end{array}$ & $\begin{array}{l}200 \\
(3 \%)\end{array}$ & $\begin{array}{c}13,487 \\
(38 \%)\end{array}$ \\
\hline $\begin{array}{l}\text { Nashville-Davidson-- } \\
\text { Murfreesboro--Franklin, TN } \\
\text { (MSA) plus Bedford, DeKalb, } \\
\text { Marshall, and Warren } \\
\text { Counties }\end{array}$ & $\begin{array}{c}40,413 \\
(75 \%)\end{array}$ & $\begin{array}{l}33,923 \\
(77 \%)\end{array}$ & $\begin{array}{c}22,117 \\
(42 \%)\end{array}$ & $\begin{array}{l}7,098 \\
(14 \%)\end{array}$ & $\begin{array}{c}1,460 \\
(3 \%)\end{array}$ & $\begin{array}{c}105,011 \\
(42 \%)\end{array}$ \\
\hline $\begin{array}{l}\text { Tullahoma-Manchester, TN } \\
(\mu S A) \text { plus Lawrence County }\end{array}$ & $\begin{array}{l}1,872 \\
(71 \%)\end{array}$ & $\begin{array}{l}1,771 \\
(59 \%)\end{array}$ & $\begin{array}{l}1,371 \\
(48 \%)\end{array}$ & $\begin{array}{l}143 \\
(4 \%)\end{array}$ & $\begin{array}{c}67 \\
(3 \%)\end{array}$ & $\begin{array}{l}5,224 \\
(35 \%)\end{array}$ \\
\hline
\end{tabular}

\section{Affordable and Available Units per 100 Tenants by AMI by Alabama Combined PUMA}

\section{Region}

\begin{tabular}{|c|c|c|c|}
\hline & $\begin{array}{l}\text { At or Below } \\
30 \% \text { AMI } \\
\text { (Extremely Low } \\
\text { Income) }\end{array}$ & $\begin{array}{l}\text { At or Below } \\
50 \% \text { AMI } \\
\text { (Extremely Low } \\
\text { Income and Very } \\
\text { Low Income) }\end{array}$ & $\begin{array}{l}\text { At or Below } \\
80 \% \text { AMI } \\
\text { (Very Low } \\
\text { Income, } \\
\text { Extremely Low } \\
\text { Income, and } \\
\text { Very Low } \\
\text { Income) }\end{array}$ \\
\hline Alexander City ( $\mu \mathrm{SA})$ & 38 & 60 & 104 \\
\hline Anniston-Oxford-Jacksonville (MSA) & 21 & 73 & 112 \\
\hline $\begin{array}{l}\text { Atmore }(\mu \mathrm{SA}) \text { plus Choctaw, Clarke, Conecuh, } \\
\text { Monroe, Washington, and Wilcox Counties }\end{array}$ & 74 & 99 & 107 \\
\hline Auburn-Opelika (MSA) & 22 & 49 & 101 \\
\hline $\begin{array}{l}\text { Birmingham-Hoover (MSA) plus Fayette, Lamar } \\
\text { Counties, and southern Marion County minus Chilton } \\
\text { and Bibb Counties }\end{array}$ & 36 & 73 & 105 \\
\hline $\begin{array}{l}\text { Columbus, GA-AL (MSA) plus Eufaula ( } \mu \mathrm{SA} \text { ) and Troy } \\
(\mu \mathrm{SA}) \text { and Bullock and Macon Counties }\end{array}$ & 44 & 72 & 107 \\
\hline Cullman $(\mu \mathrm{SA})$ plus Winston County & 46 & 91 & 102 \\
\hline Daphne-Fairhope-Foley (MSA) & 105 & 151 & 157 \\
\hline Decatur (MSA) & 55 & 99 & 108 \\
\hline Dothan (MSA) plus Ozark ( $\mu \mathrm{SA})$ & 61 & 88 & 116 \\
\hline $\begin{array}{l}\text { Enterprise ( } \mu \mathrm{SA}) \text { plus Butler, Covington, and } \\
\text { Crenshaw Counties }\end{array}$ & 38 & 102 & 109 \\
\hline $\begin{array}{l}\text { Florence-Muscle Shoals (MSA) plus Franklin County } \\
\text { and northern Marion County }\end{array}$ & 34 & 78 & 106 \\
\hline Fort Payne $(\mu S A)$ plus Scottsboro $(\mu S A)$ & 61 & 85 & 111 \\
\hline Gadsden (MSA) & 65 & 73 & 108 \\
\hline Huntsville (MSA) plus Albertville $(\mu S A)$ & 50 & 92 & 111 \\
\hline Mobile (MSA) & 36 & 58 & 115 \\
\hline
\end{tabular}




\begin{tabular}{|l|c|c|c|}
\hline Montgomery (MSA) & 30 & 61 & 104 \\
\hline $\begin{array}{l}\text { Talladega-Sylacauga }(\mu \mathrm{SA}) \text { plus Valley }(\mu \mathrm{SA}) \text { and } \\
\text { Chilton, Clay, Cleburne, and Randolph Counties }\end{array}$ & 63 & 87 & 101 \\
\hline $\begin{array}{l}\text { Tuscaloosa (MSA) plus Selma }(\mu \mathrm{SA}) \text { and Bibb, Greene, } \\
\text { Marengo, Perry, and Sumter Counties }\end{array}$ & 38 & 73 & 99 \\
\hline
\end{tabular}

\section{Affordable and Available Units per 100 Tenants by AMI by Florida Combined PUMA}

\section{Region}

\begin{tabular}{|c|c|c|c|}
\hline & $\begin{array}{l}\text { At or Below } \\
30 \% \text { AMI } \\
\text { (Extremely Low } \\
\text { Income) }\end{array}$ & $\begin{array}{l}\text { At or Below } \\
50 \% \text { AMI } \\
\text { (Extremely Low } \\
\text { Income and Very } \\
\text { Low Income) }\end{array}$ & $\begin{array}{l}\text { At or Below } \\
80 \% \text { AMI } \\
\text { (Very Low } \\
\text { Income, } \\
\text { Extremely Low } \\
\text { Income, and } \\
\text { Very Low } \\
\text { Income) }\end{array}$ \\
\hline Cape Coral-Fort Myers (MSA) & 13 & 30 & 88 \\
\hline $\begin{array}{l}\text { Crestview-Fort Walton Beach-Destin (MSA) minus } \\
\text { Walton County }\end{array}$ & 21 & 36 & 91 \\
\hline $\begin{array}{l}\text { Deltona-Daytona Beach-Ormond Beach (MSA) plus } \\
\text { Palm Coast (MSA) }\end{array}$ & 14 & 28 & 69 \\
\hline Fort Lauderdale & 15 & 17 & 50 \\
\hline Gainesville (MSA) minus Gilchrist County & 27 & 72 & 102 \\
\hline Homosassa Springs (MSA) & 5 & 41 & 100 \\
\hline Jacksonville (MSA) plus Palatka ( $\mu \mathrm{SA})$ & 33 & 47 & 99 \\
\hline $\begin{array}{l}\text { Lake City ( } \mu \text { SA) plus Bradford, Dixie, Gilchrist, } \\
\text { Hamilton, Lafayette, Levy, Madison, Suwannee, } \\
\text { Taylor, and Union Counties }\end{array}$ & 41 & 61 & 101 \\
\hline Lakeland-Winter Haven (MSA) & 24 & 37 & 78 \\
\hline Miami-Dade plus Key West ( $\mu \mathrm{SA})$ & 22 & 26 & 41 \\
\hline Naples-Immokalee-Marco Island (MSA) & 21 & 31 & 90 \\
\hline North Port-Sarasota-Bradenton (MSA) & 25 & 37 & 85 \\
\hline Ocala (MSA) & 23 & 52 & 116 \\
\hline $\begin{array}{l}\text { Orlando-Kissimmee-Sanford (MSA) plus The Villages } \\
\text { (MSA) }\end{array}$ & 14 & 24 & 79 \\
\hline Palm Bay-Melbourne-Titusville (MSA) & 26 & 52 & 106 \\
\hline $\begin{array}{l}\text { Panama City (MSA) plus Calhoun, Franklin, Gadsden, } \\
\text { Holmes, Jackson, Jefferson, Liberty, Wakulla, Walton, } \\
\text { and Washington Counties }\end{array}$ & 41 & 61 & 98 \\
\hline Pensacola-Ferry Pass-Brent (MSA) & 36 & 73 & 115 \\
\hline Port St. Lucie (MSA) & 18 & 35 & 83 \\
\hline Punta Gorda (MSA) & 13 & 26 & 89 \\
\hline
\end{tabular}




\begin{tabular}{|l|c|c|c|}
\hline Sebastian-Vero Beach (MSA) & 35 & 72 & 145 \\
\hline $\begin{array}{l}\text { Sebring (MSA) plus Arcadia, Clewiston, Okeechobee, } \\
\text { and Wauchula ( } \mu \text { SAs) and Glades County }\end{array}$ & 27 & 42 & 89 \\
\hline $\begin{array}{l}\text { Tallahassee (MSA) minus Gadsden, Jefferson, and } \\
\text { Wakulla Counties }\end{array}$ & 16 & 49 & 103 \\
\hline Tampa-St. Petersburg-Clearwater (MSA) & 23 & 33 & 88 \\
\hline West Palm Beach-Boca Raton & 16 & 29 & 71 \\
\hline
\end{tabular}

\section{Affordable and Available Units per 100 Tenants by AMI by Georgia Combined PUMA}

\section{Region}

\begin{tabular}{|c|c|c|c|}
\hline & $\begin{array}{l}\text { At or Below } \\
30 \% \text { AMI } \\
\text { (Extremely Low } \\
\text { Income) }\end{array}$ & $\begin{array}{l}\text { At or Below } \\
50 \% \text { AMI } \\
\text { (Extremely Low } \\
\text { Income and Very } \\
\text { Low Income) }\end{array}$ & $\begin{array}{l}\text { At or Below } \\
80 \% \text { AMI } \\
\text { (Very Low } \\
\text { Income, } \\
\text { Extremely Low } \\
\text { Income, and } \\
\text { Very Low } \\
\text { Income) }\end{array}$ \\
\hline $\begin{array}{l}\text { Albany (MSA) plus Bainbridge, Moultrie, and } \\
\text { Thomasville ( } \mu \text { SAs) and Calhoun, Early, Grady, Miller, } \\
\text { Mitchell, and Seminole Counties }\end{array}$ & 32 & 49 & 92 \\
\hline $\begin{array}{l}\text { Athens-Clarke County (MSA) plus Elbert and Greene } \\
\text { Counties }\end{array}$ & 21 & 38 & 90 \\
\hline $\begin{array}{l}\text { Atlanta-Sandy Springs-Roswell (MSA) minus Haralson } \\
\text { and Pickens Counties plus Jefferson, LaGrange, and } \\
\text { Thomaston ( } \mu \mathrm{SAs} \text { ) }\end{array}$ & 24 & 47 & 98 \\
\hline $\begin{array}{l}\text { Augusta-Richmond County, GA-SC (MSA) plus } \\
\text { Glascock, Jefferson, Jenkins, Taliaferro, Warren, } \\
\text { Washington, and Wilkes Counties }\end{array}$ & 26 & 51 & 105 \\
\hline $\begin{array}{l}\text { Brunswick (MSA) minus Brantley County plus St. } \\
\text { Marys ( } \mu \mathrm{SA})\end{array}$ & 29 & 66 & 101 \\
\hline Chattanooga, TN-GA (MSA) plus Summerville ( $\mu S A)$ & 22 & 68 & 108 \\
\hline $\begin{array}{l}\text { Columbus, GA-AL (MSA) plus Americus, Eufaula, AL- } \\
\text { GA, and Cordele ( } \mu \text { SAs) and Clay, Dooley, Macon, } \\
\text { Randolph, Stewart, Talbot, Taylor, and Webster } \\
\text { Counties }\end{array}$ & 33 & 63 & 91 \\
\hline $\begin{array}{l}\text { Cornelia ( } \mu \mathrm{SA} \text { ) plus Toccoa ( } \mu \mathrm{SA}) \text { plus Banks, Franklin, } \\
\text { and Hart Counties }\end{array}$ & 30 & 72 & 95 \\
\hline $\begin{array}{l}\text { Dalton (MSA) plus Calhoun ( } \mu \mathrm{SA} \text { ) plus Fannin, Gilmer, } \\
\text { and Pickens Counties }\end{array}$ & 49 & 91 & 115 \\
\hline $\begin{array}{l}\text { Dublin, GA ( } \mu \mathrm{SA} \text { ) plus Bleckley, Candler, Dodge, } \\
\text { Emanuel, Treutlen, and Wilcox Counties }\end{array}$ & 42 & 67 & 103 \\
\hline Gainesville (MSA) & 29 & 56 & 92 \\
\hline
\end{tabular}




\begin{tabular}{|l|c|c|c|}
\hline Hinesville (MSA) plus Bryan County & 24 & 44 & 77 \\
\hline $\begin{array}{l}\text { Macon-Bibb County (MSA) plus Warner Robins (MSA) } \\
\text { and Baldwin, Putnam, and Wilkinson Counties }\end{array}$ & 37 & 62 & 100 \\
\hline Northeast Georgia Nonmetropolitan Area & 53 & 76 & 110 \\
\hline $\begin{array}{l}\text { Rome (MSA) plus Cedartown ( } \mu S A) \text { and Haralson } \\
\text { County }\end{array}$ & 15 & 49 & 84 \\
\hline $\begin{array}{l}\text { Savannah (MSA) minus Bryan County plus Statesboro } \\
(\mu S A) \text { and Screven County }\end{array}$ & 26 & 43 & 87 \\
\hline $\begin{array}{l}\text { Valdosta (MSA) plus Fitzgerald, Douglas, Tifton, and } \\
\text { Waycross ( } \mu \text { SAs) and Atkinson, Bacon, Berrien, } \\
\text { Brantley, Charlton, Clinch, Cook, Irwin, and Turner } \\
\text { Counties }\end{array}$ & 27 & 52 & 103 \\
\hline $\begin{array}{l}\text { Vidalia ( } \mu \text { SA) plus Jesup ( } \mu S A) \text { and Appling, Evans, Jeff } \\
\text { Davis, Tattnall, Telfair, and Wheeler Counties }\end{array}$ & 65 & 97 & \\
\hline
\end{tabular}

\section{Affordable and Available Units per 100 Tenants by AMI by Louisiana Combined PUMA}

\section{Region}

\begin{tabular}{|c|c|c|c|}
\hline & $\begin{array}{l}\text { At or Below } \\
30 \% \text { AMI } \\
\text { (Extremely Low } \\
\text { Income) }\end{array}$ & $\begin{array}{l}\text { At or Below } \\
50 \% \text { AMI } \\
\text { (Extremely Low } \\
\text { Income and Very } \\
\text { Low Income) }\end{array}$ & $\begin{array}{l}\text { At or Below } \\
80 \% \text { AMI } \\
\text { (Very Low } \\
\text { Income, } \\
\text { Extremely Low } \\
\text { Income, and } \\
\text { Very Low } \\
\text { Income) }\end{array}$ \\
\hline $\begin{array}{l}\text { Alexandria (MSA) plus Fort Polk South ( } \mu \text { SA) and } \\
\text { Natchez, MS-LA ( } \mu \text { SA) and Avoyelles, Catahoula, } \\
\text { LaSalle, and Winn Parishes }\end{array}$ & 40 & 61 & 107 \\
\hline Baton Rouge (MSA) & 31 & 70 & 105 \\
\hline Hammond (MSA) plus Bogalusa ( $\mu \mathrm{SA})$ & 37 & 61 & 106 \\
\hline Houma-Thibodaux (MSA) plus Assumption Parish & 44 & 56 & 89 \\
\hline Lafayette (MSA) plus Morgan City ( $\mu \mathrm{SA})$ & 46 & 71 & 104 \\
\hline $\begin{array}{l}\text { Lake Charles (MSA) plus DeRidder ( } \mu \mathrm{SA}) \text { and Jennings } \\
(\mu \mathrm{SA}) \text { and Allen Parish }\end{array}$ & 40 & 69 & 103 \\
\hline $\begin{array}{l}\text { Monroe (MSA) plus Bastrop ( } \mu \text { SA) and Caldwell, East } \\
\text { Carroll, Franklin, Jackson, Madison, Richland, Tensas } \\
\text { and West Carroll Parishes }\end{array}$ & 39 & 64 & 104 \\
\hline New Orleans-Metairie (MSA) & 25 & 42 & 95 \\
\hline Opelousas ( $\mu \mathrm{SA}$ ) plus Evangeline Parish & 64 & 70 & 102 \\
\hline $\begin{array}{l}\text { Ruston ( } \mu \mathrm{SA} \text { ) plus Natchitoches ( } \mu \mathrm{SA}) \text { and DeSoto, } \\
\text { Bienville, Claiborne, Red River, and Sabine Parishes }\end{array}$ & 43 & 59 & 97 \\
\hline Shreveport-Bossier City (MSA) minus DeSoto Parish & 32 & 64 & 100 \\
\hline
\end{tabular}




\section{Affordable and Available Units per 100 Tenants by AMI by Mississippi Combined PUMA}

\section{Region}

\begin{tabular}{|c|c|c|c|}
\hline & $\begin{array}{l}\text { At or Below } \\
30 \% \text { AMI } \\
\text { (Extremely Low } \\
\text { Income) }\end{array}$ & $\begin{array}{l}\text { At or Below } \\
50 \% \text { AMI } \\
\text { (Extremely Low } \\
\text { Income and Very } \\
\text { Low Income) }\end{array}$ & $\begin{array}{l}\text { At or Below } \\
80 \% \text { AMI } \\
\text { (Very Low } \\
\text { Income, } \\
\text { Extremely Low } \\
\text { Income, and } \\
\text { Very Low } \\
\text { Income) }\end{array}$ \\
\hline $\begin{array}{l}\text { Brookhaven ( } \mu \mathrm{SA}) \text { plus McComb }(\mu \mathrm{SA}) \text { and Vicksburg } \\
(\mu \mathrm{SA})\end{array}$ & 34 & 55 & 95 \\
\hline $\begin{array}{l}\text { Cleveland ( } \mu \mathrm{SA}) \text { plus Indianola }(\mu \mathrm{SA}) \text { and Greenville } \\
(\mu \mathrm{SA})\end{array}$ & 23 & 34 & 65 \\
\hline $\begin{array}{l}\text { Columbus ( } \mu \mathrm{SA}) \text { plus Starkville }(\mu \mathrm{SA}) \text { and West Point } \\
(\mu \mathrm{SA})\end{array}$ & 36 & 59 & 96 \\
\hline Greenwood $(\mu \mathrm{SA})$ plus Grenada $(\mu \mathrm{SA})$ & 29 & 60 & 103 \\
\hline $\begin{array}{l}\text { Gulfport-Biloxi-Pascagoula (MSA) plus Picayune ( } \mu \mathrm{SA}) \\
\text { and George and Stone Counties }\end{array}$ & 30 & 56 & 107 \\
\hline Hattiesburg (MSA) plus Marion County & 37 & 61 & 102 \\
\hline Jackson (MSA) & 34 & 55 & 101 \\
\hline Laurel ( $\mu \mathrm{SA})$ & 33 & 60 & 96 \\
\hline Memphis, TN-MS-AR (MSA) & 41 & 80 & 101 \\
\hline Meridian $(\mu \mathrm{SA})$ plus Laurel $(\mu \mathrm{SA})$ & 23 & 54 & 88 \\
\hline Tupelo ( $\mu \mathrm{SA}$ ) plus Oxford ( $\mu \mathrm{SA})$ & 30 & 59 & 94 \\
\hline
\end{tabular}

\section{Affordable and Available Units per 100 Tenants by AMI by Tennessee Combined PUMA}

\section{Region}

\begin{tabular}{|c|c|c|c|}
\hline & $\begin{array}{l}\text { At or Below } \\
30 \% \text { AMI } \\
\text { (Extremely Low } \\
\text { Income) }\end{array}$ & $\begin{array}{l}\text { At or Below } \\
50 \% \text { AMI } \\
\text { (Extremely Low } \\
\text { Income and Very } \\
\text { Low Income) }\end{array}$ & $\begin{array}{c}\text { At or Below } \\
80 \% \text { AMI } \\
\text { (Very Low } \\
\text { Income, } \\
\text { Extremely Low } \\
\text { Income, and } \\
\text { Very Low } \\
\text { Income) }\end{array}$ \\
\hline $\begin{array}{l}\text { Brownsville ( } \mu \mathrm{SA} \text { ) plus Decatur, Hardeman, Hardin, } \\
\text { Henderson, and McNairy Counties }\end{array}$ & 60 & 70 & 101 \\
\hline $\begin{array}{l}\text { Chattanooga, TN-GA (MSA) plus Bledsoe, Grundy, } \\
\text { Meigs, and Rhea Counties }\end{array}$ & 40 & 77 & 111 \\
\hline Clarksville, TN-KY (MSA) plus Stewart County & 46 & 67 & 110 \\
\hline Cleveland (MSA) plus McMinn County & 31 & 60 & 101 \\
\hline
\end{tabular}




\begin{tabular}{|c|c|c|c|}
\hline Cookeville ( $\mu \mathrm{SA}$ ) plus Clay and Picket Counties & 23 & 43 & 91 \\
\hline $\begin{array}{l}\text { Crossville ( } \mu \mathrm{SA} \text { ) plus Fentress, Van Buren, and White } \\
\text { Counties }\end{array}$ & 30 & 70 & 98 \\
\hline Jackson (MSA) plus Crockett, Dyer, and Lake Counties & 52 & 81 & 107 \\
\hline $\begin{array}{l}\text { Johnson City (MSA) plus Johnson and Greene } \\
\text { Counties }\end{array}$ & 23 & 66 & 97 \\
\hline Kingsport-Bristol-Bristol, TN-VA (MSA) & 52 & 77 & 108 \\
\hline $\begin{array}{l}\text { Knoxville (MSA) plus Claiborne, Hancock, Monroe, } \\
\text { and Scott Counties minus Grainger County }\end{array}$ & 46 & 71 & 102 \\
\hline $\begin{array}{l}\text { Lawrenceburg ( } \mu \mathrm{SA}) \text { plus Giles, Lewis, Perry, and } \\
\text { Wayne Counties }\end{array}$ & 35 & 63 & 95 \\
\hline $\begin{array}{l}\text { Martin ( } \mu \mathrm{SA}) \text { plus Paris }(\mu \mathrm{SA}) \text { and Benton, Carroll, } \\
\text { Houston, and Humphreys Counties }\end{array}$ & 25 & 57 & 106 \\
\hline Memphis, TN-MS-AR (MSA) plus Lauderdale County & 27 & 60 & 103 \\
\hline $\begin{array}{l}\text { Morristown (MSA) plus Cocke, Grainger, and Sevier } \\
\text { Counties }\end{array}$ & 45 & 69 & 102 \\
\hline $\begin{array}{l}\text { Nashville-Davidson--Murfreesboro--Franklin, TN } \\
\text { (MSA) plus Bedford, DeKalb, Marshall, and Warren } \\
\text { Counties }\end{array}$ & 38 & 58 & 97 \\
\hline $\begin{array}{l}\text { Tullahoma-Manchester, TN ( } \mu \mathrm{SA}) \text { plus Lawrence } \\
\text { County }\end{array}$ & 52 & 80 & 99 \\
\hline
\end{tabular}

\section{Surplus or Deficit of Affordable and Available Units by AMI by Alabama Combined PUMA}

\section{Region}

\begin{tabular}{|c|c|c|c|}
\hline & $\begin{array}{l}\text { At or Below } \\
30 \% \text { AMI } \\
\text { (Extremely Low } \\
\text { Income) }\end{array}$ & $\begin{array}{l}\text { At or Below } \\
50 \% \text { AMI } \\
\text { (Extremely Low } \\
\text { Income and } \\
\text { Very Low } \\
\text { Income) }\end{array}$ & $\begin{array}{c}\text { At or Below } 80 \% \\
\text { AMI } \\
\text { (Very Low Income, } \\
\text { Extremely Low } \\
\text { Income, and Very } \\
\text { Low Income) }\end{array}$ \\
\hline Alexander City ( $\mu \mathrm{SA})$ & $-2,461$ & $-2,820$ & 379 \\
\hline Anniston-Oxford-Jacksonville (MSA) & $-3,152$ & $-1,680$ & 1,086 \\
\hline $\begin{array}{l}\text { Atmore }(\mu \mathrm{SA}) \text { plus Choctaw, Clarke, Conecuh, } \\
\text { Monroe, Washington, and Wilcox Counties }\end{array}$ & $-1,647$ & -94 & 737 \\
\hline Auburn-Opelika (MSA) & $-7,114$ & $-6,684$ & 144 \\
\hline $\begin{array}{l}\text { Birmingham-Hoover (MSA) plus Fayette, Lamar } \\
\text { Counties, and southern Marion County minus Chilton } \\
\text { and Bibb Counties }\end{array}$ & $-25,895$ & $-17,985$ & 4,594 \\
\hline $\begin{array}{l}\text { Columbus, GA-AL (MSA) plus Eufaula ( } \mu \mathrm{SA}) \text { and Troy } \\
(\mu \mathrm{SA}) \text { and Bullock and Macon Counties }\end{array}$ & $-3,782$ & $-2,863$ & 1,008 \\
\hline Cullman $(\mu \mathrm{SA})$ plus Winston County & -950 & -246 & 110 \\
\hline Daphne-Fairhope-Foley (MSA) & 142 & 4,107 & 6,907 \\
\hline
\end{tabular}




\begin{tabular}{|l|c|c|c|}
\hline Decatur (MSA) & $-2,142$ & -92 & 996 \\
\hline Dothan (MSA) plus Ozark $(\mu S A)$ & $-2,308$ & $-1,142$ & 2,319 \\
\hline $\begin{array}{l}\text { Enterprise }(\mu S A) \text { plus Butler, Covington, and } \\
\text { Crenshaw Counties }\end{array}$ & $-2,153$ & 107 & 883 \\
\hline $\begin{array}{l}\text { Florence-Muscle Shoals (MSA) plus Franklin County } \\
\text { and northern Marion County }\end{array}$ & $-4,278$ & $-2,456$ & 942 \\
\hline Fort Payne $(\mu S A)$ plus Scottsboro $(\mu S A)$ & $-1,090$ & -812 & 903 \\
\hline Gadsden (MSA) & -695 & $-1,006$ & 447 \\
\hline Huntsville (MSA) plus Albertville $(\mu S A)$ & $-8,234$ & $-2,400$ & 4,753 \\
\hline Mobile (MSA) & $-7,998$ & $-10,133$ & 5,371 \\
\hline Montgomery $(M S A)$ & $-10,907$ & $-9,922$ & 1,497 \\
\hline $\begin{array}{l}\text { Talladega-Sylacauga ( } \mu S A) \text { plus Valley ( } \mu S A) \text { and } \\
\text { Chilton, Clay, Cleburne, and Randolph Counties }\end{array}$ & $-1,618$ & $-1,110$ & 164 \\
\hline $\begin{array}{l}\text { Tuscaloosa (MSA) plus Selma ( } \mu S A) \text { and Bibb, Greene, } \\
\text { Marengo, Perry, and Sumter Counties }\end{array}$ & $-8,311$ & $-6,107$ & -459 \\
\hline
\end{tabular}

\section{Surplus or Deficit of Affordable and Available Units by AMI by Florida Combined PUMA}

\section{Region}

\begin{tabular}{|c|c|c|c|}
\hline & $\begin{array}{l}\text { At or Below } \\
30 \% \text { AMI } \\
\text { (Extremely Low } \\
\text { Income) }\end{array}$ & $\begin{array}{l}\text { At or Below } \\
50 \% \text { AMI } \\
\text { (Extremely Low } \\
\text { Income and } \\
\text { Very Low } \\
\text { Income) }\end{array}$ & $\begin{array}{l}\text { At or Below } 80 \% \\
\text { AMI } \\
\text { (Very Low Income, } \\
\text { Extremely Low } \\
\text { Income, and Very } \\
\text { Low Income) }\end{array}$ \\
\hline Cape Coral-Fort Myers (MSA) & $-12,853$ & $-20,122$ & $-5,597$ \\
\hline $\begin{array}{l}\text { Crestview-Fort Walton Beach-Destin (MSA) minus } \\
\text { Walton County }\end{array}$ & $-1,926$ & $-5,203$ & $-1,387$ \\
\hline $\begin{array}{l}\text { Deltona-Daytona Beach-Ormond Beach (MSA) plus } \\
\text { Palm Coast (MSA) }\end{array}$ & $-10,403$ & $-17,081$ & $-13,003$ \\
\hline Fort Lauderdale & $-34,974$ & $-68,662$ & $-67,165$ \\
\hline Gainesville (MSA) minus Gilchrist County & $-10,708$ & $-6,099$ & 484 \\
\hline Homosassa Springs (MSA) & $-1,063$ & $-2,037$ & 6 \\
\hline Jacksonville (MSA) plus Palatka ( $\mu \mathrm{SA})$ & $-29,913$ & $-40,184$ & $-1,328$ \\
\hline $\begin{array}{l}\text { Lake City }(\mu \mathrm{SA}) \text { plus Bradford, Dixie, Gilchrist, } \\
\text { Hamilton, Lafayette, Levy, Madison, Suwannee, } \\
\text { Taylor, and Union Counties }\end{array}$ & $-4,176$ & $-4,524$ & 101 \\
\hline Lakeland-Winter Haven (MSA) & $-9,399$ & $-14,823$ & $-9,267$ \\
\hline Miami-Dade plus Key West ( $\mu S A)$ & $-64,861$ & $-113,874$ & $-140,351$ \\
\hline Naples-Immokalee-Marco Island (MSA) & $-3,444$ & $-7,639$ & $-2,124$ \\
\hline North Port-Sarasota-Bradenton (MSA) & $-9,824$ & $-18,553$ & $-7,455$ \\
\hline Ocala (MSA) & $-4,719$ & $-5,041$ & 2,708 \\
\hline
\end{tabular}




\begin{tabular}{|l|c|c|c|}
\hline $\begin{array}{l}\text { Orlando-Kissimmee-Sanford (MSA) plus The Villages } \\
\text { (MSA) }\end{array}$ & $-45,055$ & $-86,356$ & $-39,466$ \\
\hline Palm Bay-Melbourne-Titusville (MSA) & $-8,326$ & $-11,080$ & 2,403 \\
\hline $\begin{array}{l}\text { Panama City (MSA) plus Calhoun, Franklin, Gadsden, } \\
\text { Holmes, Jackson, Jefferson, Liberty, Wakulla, Walton, } \\
\text { and Washington Counties }\end{array}$ & $-7,159$ & $-7,904$ & -644 \\
\hline Pensacola-Ferry Pass-Brent (MSA) & $-6,627$ & $-5,216$ & 5,053 \\
\hline Port St. Lucie (MSA) & $-6,975$ & $-10,885$ & $-4,428$ \\
\hline Punta Gorda (MSA) & $-2,322$ & $-3,722$ & $-1,226$ \\
\hline Sebastian-Vero Beach (MSA) & $-1,399$ & $-1,228$ & 3,233 \\
\hline $\begin{array}{l}\text { Sebring (MSA) plus Arcadia, Clewiston, Okeechobee, } \\
\text { and Wauchula ( } \mu \text { SAs) and Glades County }\end{array}$ & $-2,719$ & $-4,521$ & $-1,611$ \\
\hline $\begin{array}{l}\text { Tallahassee (MSA) minus Gadsden, Jefferson, and } \\
\text { Wakulla Counties }\end{array}$ & $-13,225$ & $-13,324$ & 1,290 \\
\hline Tampa-St. Petersburg-Clearwater (MSA) & $-60,783$ & $-98,686$ & $-28,022$ \\
\hline West Palm Beach-Boca Raton & $-31,360$ & $-47,284$ & $-30,265$ \\
\hline
\end{tabular}

\section{Surplus or Deficit of Affordable and Available Units by AMI by Georgia Combined PUMA}

\section{Region}

\begin{tabular}{|c|c|c|c|}
\hline & $\begin{array}{l}\text { At or Below } \\
30 \% \text { AMI } \\
\text { (Extremely Low } \\
\text { Income) }\end{array}$ & $\begin{array}{l}\text { At or Below } \\
50 \% \text { AMI } \\
\text { (Extremely Low } \\
\text { Income and } \\
\text { Very Low } \\
\text { Income) }\end{array}$ & $\begin{array}{l}\text { At or Below } 80 \% \\
\text { AMI } \\
\text { (Very Low Income, } \\
\text { Extremely Low } \\
\text { Income, and Very } \\
\text { Low Income) } \\
\end{array}$ \\
\hline $\begin{array}{l}\text { Albany (MSA) plus Bainbridge, Moultrie, and } \\
\text { Thomasville ( } \mu \text { SAs) and Calhoun, Early, Grady, Miller, } \\
\text { Mitchell, and Seminole Counties }\end{array}$ & $-8,760$ & $-10,757$ & $-2,308$ \\
\hline $\begin{array}{l}\text { Athens-Clarke County (MSA) plus Elbert and Greene } \\
\text { Counties }\end{array}$ & $-10,379$ & $-12,349$ & $-2,604$ \\
\hline $\begin{array}{l}\text { Atlanta-Sandy Springs-Roswell (MSA) minus Haralson } \\
\text { and Pickens Counties plus Jefferson, LaGrange, and } \\
\text { Thomaston ( } \mu \mathrm{SAs} \text { ) }\end{array}$ & $-127,604$ & $-160,953$ & $-10,695$ \\
\hline $\begin{array}{l}\text { Augusta-Richmond County, GA-SC (MSA) plus } \\
\text { Glascock, Jefferson, Jenkins, Taliaferro, Warren, } \\
\text { Washington, and Wilkes Counties }\end{array}$ & $-11,118$ & $-12,322$ & 1,739 \\
\hline $\begin{array}{l}\text { Brunswick (MSA) minus Brantley County plus St. } \\
\text { Marys ( } \mu S A)\end{array}$ & $-3,328$ & $-3,196$ & 167 \\
\hline Chattanooga, TN-GA (MSA) plus Summerville ( $\mu S A)$ & $-2,789$ & $-2,525$ & 794 \\
\hline $\begin{array}{l}\text { Columbus, GA-AL (MSA) plus Americus, Eufaula, AL- } \\
\text { GA, and Cordele ( } \mu \text { SAs) and Clay, Dooley, Macon, } \\
\text { Randolph, Stewart, Talbot, Taylor, and Webster } \\
\text { Counties }\end{array}$ & $-9,493$ & $-8,961$ & $-3,090$ \\
\hline
\end{tabular}




\begin{tabular}{|l|c|c|c|}
\hline $\begin{array}{l}\text { Cornelia }(\mu S A) \text { plus Toccoa }(\mu S A) \text { and Banks, Franklin, } \\
\text { and Hart Counties }\end{array}$ & $-2,572$ & $-1,702$ & -437 \\
\hline $\begin{array}{l}\text { Dalton (MSA) plus Calhoun ( } \mu \text { SA) and Fannin, Gilmer, } \\
\text { and Pickens Counties }\end{array}$ & $-2,505$ & -981 & 3,124 \\
\hline $\begin{array}{l}\text { Dublin, GA ( } \mu \text { SA) plus Bleckley, Candler, Dodge, } \\
\text { Emanuel, Treutlen, and Wilcox Counties }\end{array}$ & $-2,241$ & $-2,305$ & 329 \\
\hline Gainesville (MSA) & $-3,300$ & $-4,021$ & -957 \\
\hline Hinesville (MSA) plus Bryan County & $-2,814$ & $-2,971$ & $-2,379$ \\
\hline $\begin{array}{l}\text { Macon-Bibb County (MSA) plus Warner Robins (MSA) } \\
\text { and Baldwin, Putnam, and Wilkinson Counties }\end{array}$ & $-13,477$ & $-12,592$ & -207 \\
\hline Northeast Georgia Nonmetropolitan Area & $-1,319$ & $-1,270$ & 825 \\
\hline $\begin{array}{l}\text { Rome (MSA) plus Cedartown ( } \mu \text { SA) plus Haralson } \\
\text { County }\end{array}$ & $-4,381$ & $-5,054$ & 88 \\
\hline $\begin{array}{l}\text { Savannah (MSA) minus Bryan County plus Statesboro } \\
(\mu S A) \text { and Screven County }\end{array}$ & $-11,991$ & $-16,344$ & $-6,926$ \\
\hline $\begin{array}{l}\text { Valdosta (MSA) plus Fitzgerald, Douglas, Tifton, and } \\
\text { Waycross }(\mu S A s) \text { plus Atkinson, Bacon, Berrien, } \\
\text { Brantley, Charlton, Clinch, Cook, Irwin, and Turner } \\
\text { Counties }\end{array}$ & $-7,846$ & $-10,179$ & $-4,305$ \\
\hline $\begin{array}{l}\text { Vidalia ( } \mu S A) \text { plus Jesup ( } \mu S A) \text { plus Appling, Evans, } \\
\text { Jeff Davis, Tattnall, Telfair, and Wheeler Counties }\end{array}$ & $-1,523$ & -207 & 350 \\
\hline
\end{tabular}

\section{Surplus or Deficit of Affordable and Available Units by AMI by Louisiana Combined PUMA}

\section{Region}

\begin{tabular}{|c|c|c|c|}
\hline & $\begin{array}{l}\text { At or Below } \\
30 \% \text { AMI } \\
\text { (Extremely Low } \\
\text { Income) }\end{array}$ & $\begin{array}{l}\text { At or Below } \\
50 \% \text { AMI } \\
\text { (Extremely Low } \\
\text { Income and } \\
\text { Very Low } \\
\text { Income) }\end{array}$ & $\begin{array}{l}\text { At or Below } 80 \% \\
\text { AMI } \\
\text { (Very Low Income, } \\
\text { Extremely Low } \\
\text { Income, and Very } \\
\text { Low Income) }\end{array}$ \\
\hline $\begin{array}{l}\text { Alexandria (MSA) plus Fort Polk South ( } \mu \mathrm{SA}) \text { and } \\
\text { Natchez, MS-LA ( } \mu \mathrm{SA}) \text { and Avoyelles, Catahoula, } \\
\text { LaSalle, and Winn Parishes }\end{array}$ & $-5,135$ & $-6,650$ & 1,671 \\
\hline Baton Rouge (MSA) & $-18,063$ & $-13,550$ & 3,438 \\
\hline Hammond (MSA) plus Bogalusa ( $\mu \mathrm{SA})$ & $-5,251$ & $-5,499$ & 914 \\
\hline Houma-Thibodaux (MSA) plus Assumption Parish & $-1,898$ & $-3,958$ & $-1,572$ \\
\hline Lafayette (MSA) plus Morgan City ( $\mu \mathrm{SA}$ ) & $-9,103$ & $-8,494$ & 1,595 \\
\hline $\begin{array}{l}\text { Lake Charles (MSA) plus DeRidder ( } \mu \mathrm{SA}) \text { and Jennings } \\
(\mu \mathrm{SA}) \text { and Allen Parish }\end{array}$ & $-4,101$ & $-4,115$ & 694 \\
\hline $\begin{array}{l}\text { Monroe (MSA) plus Bastrop ( } \mu \mathrm{SA} \text { ) and Caldwell, East } \\
\text { Carroll, Franklin, Jackson, Madison, Richland, Tensas, } \\
\text { and West Carroll Parishes }\end{array}$ & $-7,918$ & $-7,550$ & 1,237 \\
\hline New Orleans-Metairie (MSA) & $-38,019$ & $-48,273$ & $-6,264$ \\
\hline
\end{tabular}




\begin{tabular}{|l|c|c|c|}
\hline Opelousas $(\mu \mathrm{SA})$ plus Evangeline Parish & -952 & $-1,822$ & 160 \\
\hline $\begin{array}{l}\text { Ruston }(\mu \mathrm{SA}) \text { plus Natchitoches }(\mu \mathrm{SA}) \text { and DeSoto, } \\
\text { Bienville, Claiborne, Red River, and Sabine Parishes }\end{array}$ & $-4,923$ & $-5,613$ & -579 \\
\hline Shreveport-Bossier City (MSA) minus DeSoto Parish & $-11,431$ & $-9,962$ & 72 \\
\hline
\end{tabular}

\section{Surplus or Deficit of Affordable and Available Units by AMI by Mississippi Combined PUMA Region}

\begin{tabular}{|c|c|c|c|}
\hline & $\begin{array}{l}\text { At or Below } \\
30 \% \text { AMI } \\
\text { (Extremely Low } \\
\text { Income) }\end{array}$ & $\begin{array}{l}\text { At or Below } \\
50 \% \text { AMI } \\
\text { (Extremely Low } \\
\text { Income and } \\
\text { Very Low } \\
\text { Income) }\end{array}$ & $\begin{array}{c}\text { At or Below } 80 \% \\
\text { AMI } \\
\text { (Very Low Income, } \\
\text { Extremely Low } \\
\text { Income, and Very } \\
\text { Low Income) } \\
\end{array}$ \\
\hline $\begin{array}{l}\text { Brookhaven }(\mu \mathrm{SA}) \text { plus McComb }(\mu \mathrm{SA}) \text { and Vicksburg } \\
(\mu \mathrm{SA})\end{array}$ & $-3,160$ & $-3,743$ & -548 \\
\hline $\begin{array}{l}\text { Cleveland }(\mu \mathrm{SA}) \text { plus Indianola }(\mu \mathrm{SA}) \text { and Greenville } \\
(\mu \mathrm{SA})\end{array}$ & $-2,825$ & $-5,058$ & $-4,356$ \\
\hline $\begin{array}{l}\text { Columbus ( } \mu \mathrm{SA}) \text { plus Starkville }(\mu \mathrm{SA}) \text { and West Point } \\
(\mu \mathrm{SA})\end{array}$ & $-4,983$ & $-4,412$ & -675 \\
\hline Greenwood $(\mu \mathrm{SA})$ plus Grenada $(\mu \mathrm{SA})$ & $-3,723$ & $-3,189$ & 336 \\
\hline $\begin{array}{l}\text { Gulfport-Biloxi-Pascagoula (MSA) plus Picayune ( } \mu \mathrm{SA}) \\
\text { and George and Stone Counties }\end{array}$ & $-9,348$ & $-10,952$ & 2,491 \\
\hline Hattiesburg (MSA) plus Marion County & $-3,135$ & $-4,420$ & 254 \\
\hline Jackson (MSA) & $-13,380$ & $-15,268$ & 307 \\
\hline Laurel $(\mu \mathrm{SA})$ & $-1,723$ & $-1,906$ & -286 \\
\hline Memphis, TN-MS-AR (MSA) & $-6,540$ & $-3,795$ & 217 \\
\hline Meridian ( $\mu \mathrm{SA})$ plus Laurel ( $\mu \mathrm{SA})$ & $-3,589$ & $-4,365$ & $-1,782$ \\
\hline Tupelo ( $\mu \mathrm{SA})$ plus Oxford ( $\mu \mathrm{SA})$ & $-7,234$ & $-6,728$ & $-1,398$ \\
\hline
\end{tabular}

\section{Surplus or Deficit of Affordable and Available Units by AMI by Tennessee Combined PUMA Region}

\begin{tabular}{|c|c|c|c|}
\hline & $\begin{array}{l}\text { At or Below } \\
30 \% \text { AMI } \\
\text { (Extremely Low } \\
\text { Income) }\end{array}$ & $\begin{array}{l}\text { At or Below } \\
50 \% \text { AMI } \\
\text { (Extremely Low } \\
\text { Income and } \\
\text { Very Low } \\
\text { Income) }\end{array}$ & $\begin{array}{c}\text { At or Below } 80 \% \\
\text { AMI } \\
\text { (Very Low Income, } \\
\text { Extremely Low } \\
\text { Income, and Very } \\
\text { Low Income) }\end{array}$ \\
\hline $\begin{array}{l}\text { Brownsville ( } \mu \text { SA) plus Decatur, Hardeman, Hardin, } \\
\text { Henderson, and McNairy Counties }\end{array}$ & $-1,226$ & $-1,803$ & 59 \\
\hline
\end{tabular}




\begin{tabular}{|l|c|c|c|}
\hline $\begin{array}{l}\text { Chattanooga, TN-GA (MSA) plus Bledsoe, Grundy, } \\
\text { Meigs, and Rhea Counties }\end{array}$ & $-9,553$ & $-6,158$ & 4,508 \\
\hline Clarksville, TN-KY (MSA) plus Stewart County & $-2,403$ & $-2,645$ & 1,661 \\
\hline Cleveland (MSA) plus McMinn County & $-4,510$ & $-4,310$ & 158 \\
\hline Cookeville ( $\mu$ SA) plus Clay and Picket Counties & $-2,858$ & $-3,578$ & -899 \\
\hline $\begin{array}{l}\text { Crossville ( } \mu \text { SA) plus Fentress, Van Buren, and White } \\
\text { Counties }\end{array}$ & $-1,743$ & $-1,140$ & -154 \\
\hline Jackson (MSA) plus Crockett, Dyer, and Lake Counties & $-3,777$ & $-2,579$ & 1,445 \\
\hline $\begin{array}{l}\text { Johnson City (MSA) plus Johnson and Greene } \\
\text { Counties }\end{array}$ & $-7,078$ & $-5,038$ & -708 \\
\hline Kingsport-Bristol-Bristol, TN-VA (MSA) & $-3,174$ & $-2,558$ & 1,275 \\
\hline $\begin{array}{l}\text { Knoxville (MSA) plus Claiborne, Hancock, Monroe, } \\
\text { and Scott Counties minus Grainger County }\end{array}$ & $-18,392$ & $-16,307$ & 1,628 \\
\hline $\begin{array}{l}\text { Lawrenceburg ( } \mu S A) \text { plus Giles, Lewis, Perry, and } \\
\text { Wayne Counties }\end{array}$ & $-1,410$ & $-1,453$ & -354 \\
\hline $\begin{array}{l}\text { Martin ( } \mu \text { SA) plus Paris ( } \mu S A) \text { and Benton, Carroll, } \\
\text { Houston, and Humphreys Counties }\end{array}$ & $-2,510$ & $-2,919$ & 614 \\
\hline Memphis, TN-MS-AR (MSA) plus Lauderdale County & $-31,017$ & $-29,187$ & 3,777 \\
\hline $\begin{array}{l}\text { Morristown (MSA) plus Cocke, Grainger, and Sevier } \\
\text { Counties }\end{array}$ & $-4,356$ & $-4,272$ & 395 \\
\hline $\begin{array}{l}\text { Nashville-Davidson--Murfreesboro--Franklin, TN } \\
\text { (MSA) plus Bedford, DeKalb, Marshall, and Warren } \\
\text { Counties }\end{array}$ & $-33,747$ & $-40,844$ & $-3,810$ \\
\hline $\begin{array}{l}\text { Tullahoma-Manchester, TN ( } \mu S A) \text { plus Lawrence } \\
\text { County }\end{array}$ & $-1,261$ & $-1,151$ & -43 \\
\hline
\end{tabular}

\section{References}

An, B., Bostic, R. W., Jakabovics, A., Orlando, A. W., \& Rodnyansky, S. (2017). Understanding the Small and Medium Multifamily Housing Stock. Retrieved from Enterprise Community Partners, Washington, DC: https://www.enterprisecommunity.org/download?fid=7818\&nid=3521

Center for Community Change. (2016). Opening Doors to Homes for All: The 2016 Housing Trust Fund Survey Report. Retrieved from http://housingtrustfundproject.org/wpcontent/uploads/2016/10/HTF Survey-Report-2016-final.pdf

Davis, J. E. (2006). Shared Equity Homeownership: The Changing Landscape of Resale-Restricted, OwnerOccupied Housing. Retrieved from National Housing Institute: http://cltnetwork.org/wpcontent/uploads/2014/01/2006-Shared-Equity-Homeownership.pdf

Glover, R. L., Carpenter, A., \& Duckworth, R. (2017). "Developing Inclusive Communities: Challenges and Opportunities for Mixed-Income Housing." Federal Reserve Bank of Atlanta Community and Economic Development Discussion Paper 01-17, June. Retrieved from Federal Reserve Bank of Atlanta: https://www.frbatlanta.org/-/media/documents/communitydevelopment/publications/discussion-papers/2017/01-developing-inclusive-communitieschallenges-and-opportunities-for-mixed-income-housing-2017-06-07.pdf

Immergluck, D., Carpenter, A., \& Lueders, A. (2016). "Declines in Low-Cost Rented Housing Units in Eight Large Southeastern Cities." Federal Reserve Bank of Atlanta Community and Economic 
Development Discussion Paper 03-16, May. Retrieved from Federal Reserve Bank of Atlanta: https://www.frbatlanta.org/-/media/documents/communitydevelopment/publications/discussion-papers/2016/03-housing-declines-in-low-cost-rentedhousing-units-in-eight-large-southeastern-cities-2016-05-10.pdf

Kingsley, G. T. (2017). Trends in Housing Problems and Federal Housing Assistance. Retrieved from Washington, DC: https://www.urban.org/sites/default/files/publication/94146/trends-inhousing-problems-and-federal-housing-assistance.pdf

National Housing Preservation Database. (2018). State Preservation Profiles. Retrieved from http://preservationdatabase.org/reports/2017-preservation-profiles/

Reid, C. (2018). The Links Between Affordable Housing and Economic Mobility: The Experiences of Residents Living in Low-Income Housing Tax Credit Properties. Retrieved from University of California-Berkeley, Terner Center for Housing Innovation:

http://ternercenter.berkeley.edu/uploads/Links Between Affordable Housing and Economic Mobility .pdf

Schreiber, M. (2018). Proactive Preservation of Unsubsidized Affordable Housing in Emerging Markets: Lessons from Atlanta, Cleveland, and Philadelphia. Retrieved from Joint Center for Housing Studies of Harvard University, Cambridge, MA:

Shimberg Center for Housing Studies. (2013). 2013 Rental Market Study. Retrieved from Shimberg Center for Housing Studies, University of Florida: https://www.floridahousing.org/docs/defaultsource/press/newsroom/publications/rental-housing/rentalhousing2013/2013-generalstudy.pdf?sfvrsn=2

Shimberg Center for Housing Studies. (2016). 2016 Rental Market Study. Retrieved from Shimberg Center for Housing Studies, University of Florida: https://www.floridahousing.org/docs/defaultsource/press/newsroom/publications/rental-housing/rentalhousing2016/full-rms-finalrev09 16.pdf?sfvrsn=2

Spotts, M. A., Hale-Case, G., \& Abu-Khalaf, A. (2017). Public Benefit from Publicly Owned Parcels: Effective Practices in Affordable Housing Development. Retrieved from Enterprise Community Partners, Washington, DC: https://www.enterprisecommunity.org/download?fid=3257\&nid=3739

Thaden, E., \& Wang, R. (2017). Inclusionary Housing in the United States: Prevalence, Impact, and Practices. Retrieved from the Lincoln Institute of Land Use Policy, Cambridge, MA: https://www.lincolninst.edu/sites/default/files/pubfiles/thaden wp17et1 $0 . p d f$

Williams, S., et al. (2016). The Economics of Inclusionary Development. Retrieved from the Urban Land Institute, Washington, DC: https://uli.org/wp-content/uploads/ULI-Documents/Economics-ofInclusionary-Zoning.pdf

Woo, A., \& Joh, K. (2015). "Beyond Anecdotal Evidence: Do Subsidized Housing Developments Increase Neighborhood Crime?" Applied Geography, 64, 87-96. 